ANALYSIS \& PDE

Volume $5 \quad$ No. $1 \quad 2012$

ANDRÁS VASY

THE WAVE EQUATION ON ASYMPTOTICALLY ANTI DE SITTER SPACES 


\title{
THE WAVE EQUATION ON ASYMPTOTICALLY ANTI DE SITTER SPACES
}

\begin{abstract}
ANDRÁS VASY
In this paper we describe the behavior of solutions of the Klein-Gordon equation, $\left(\square_{g}+\lambda\right) u=f$, on Lorentzian manifolds $\left(X^{\circ}, g\right)$ that are anti de Sitter-like (AdS-like) at infinity. Such manifolds are Lorentzian analogues of the so-called Riemannian conformally compact (or asymptotically hyperbolic) spaces, in the sense that the metric is conformal to a smooth Lorentzian metric $\hat{g}$ on $X$, where $X$ has a nontrivial boundary, in the sense that $g=x^{-2} \hat{g}$, with $x$ a boundary defining function. The boundary is conformally timelike for these spaces, unlike asymptotically de Sitter spaces studied before by Vasy and Baskin, which are similar but with the boundary being conformally spacelike.

Here we show local well-posedness for the Klein-Gordon equation, and also global well-posedness under global assumptions on the (null)bicharacteristic flow, for $\lambda$ below the Breitenlohner-Freedman bound, $(n-1)^{2} / 4$. These have been known before under additional assumptions. Further, we describe the propagation of singularities of solutions and obtain the asymptotic behavior (at $\partial X$ ) of regular solutions. We also define the scattering operator, which in this case is an analogue of the hyperbolic Dirichletto-Neumann map. Thus, it is shown that below the Breitenlohner-Freedman bound, the Klein-Gordon equation behaves much like it would for the conformally related metric, $\hat{g}$, with Dirichlet boundary conditions, for which propagation of singularities was shown by Melrose, Sjöstrand and Taylor, though the precise form of the asymptotics is different.
\end{abstract}

\section{Introduction}

In this paper we consider asymptotically anti de Sitter (AdS) type metrics on $n$-dimensional manifolds with boundary $X$ for $n \geq 2$. We recall the actual definition of AdS space below, but for our purposes the most important feature is the asymptotic form of the metric on these spaces, so we start by making a bold general definition. Thus, an asymptotically AdS type space is a manifold with boundary $X$ such that $X^{\circ}$ is equipped with a pseudo-Riemannian metric $g$ of signature $(1, n-1)$ that near the boundary $Y$ of $X$ is of the form

$$
g=\frac{-d x^{2}+h}{x^{2}}
$$

where $h$ is a smooth symmetric 2-cotensor on $X$ such that $X=Y \times[0, \epsilon)_{x}$ with respect to some product decomposition of $X$ near $Y$, and $\left.h\right|_{Y}$ is a section of $T^{*} Y \otimes T^{*} Y$ (rather than merely ${ }^{1} T_{Y}^{*} X \otimes T_{Y}^{*} X$ ) and is a

This work is partially supported by the National Science Foundation under grant DMS-0801226, and a Chambers Fellowship from Stanford University.

MSC2000: 35L05, 58J45.

Keywords: asymptotics, wave equation, anti de Sitter space, propagation of singularities.

${ }^{1}$ In fact, even this most general setting would necessitate only minor changes, except that the "smooth asymptotics" of Proposition 8.10 would have variable order, and the restrictions on $\lambda$ that arise here, $\lambda<(n-1)^{2} / 4$, would have to be modified. 
Lorentzian metric on $Y$ (with signature $(1, n-2)$ ). Note that $Y$ is timelike with respect to the conformal metric

$$
\hat{g}=x^{2} g, \quad \text { so } \hat{g}=-d x^{2}+h \text { near } Y,
$$

that is, the dual metric $\hat{G}$ of $\hat{g}$ is negative definite on $N^{*} Y$, that is, on $\operatorname{span}\{d x\}$, in contrast with the asymptotically de Sitter-like setting studied in [Vasy 2010b] when the boundary is spacelike. Moreover, $Y$ is not assumed to be compact; indeed, under the assumption (TF) below, which is useful for global well-posedness of the wave equation, it never is. Let the wave operator $\square=\square_{g}$ be the Laplace-Beltrami operator associated to this metric, and let

$$
P=P(\lambda)=\square_{g}+\lambda
$$

be the Klein-Gordon operator, where $\lambda \in \mathbb{C}$. The convention with the positive sign for the "spectral parameter" $\lambda$ preserves the sign of $\lambda$ relative to the $d x^{2}$ component of the metric in both the Riemannian conformally compact and the Lorentzian de Sitter-like cases, and hence is convenient when describing the asymptotics. We remark that if $n=2$ then up to a change of the (overall) sign of the metric, these spaces are asymptotically de Sitter, and hence the results of [Vasy 2010b] apply. However, some of the results are different even then, since in the two settings the role of the time variable is reversed, so the formulation of the results differs as the role of "initial" and "boundary" conditions changes.

These asymptotically AdS metrics are also analogues of the Riemannian 'conformally compact', or asymptotically hyperbolic, metrics, introduced by Mazzeo and Melrose [1987] in this form, which are of the form $x^{-2}\left(d x^{2}+h\right)$ with $d x^{2}+h$ smooth Riemannian on $X$, and $\left.h\right|_{Y}$ a section of $T^{*} Y \otimes T^{*} Y$. These have been studied extensively, in part due to the connection to AdS metrics (so some phenomena might be expected to be similar for AdS and asymptotically hyperbolic metrics) and their Riemannian signature, which makes the analysis of related PDE easier. We point out that hyperbolic space actually solves the Riemannian version of Einstein's equations, while de Sitter and anti de Sitter space satisfy the actual hyperbolic Einstein equations. We refer to [Fefferman and Graham 1985; Graham and Lee 1991; Anderson 2008] among others for analysis on conformally compact spaces. We also refer to [Witten 1998; Graham and Witten 1999; Graham and Zworski 2003] and references therein for results in the Riemannian setting that are of physical relevance. There is also a large body of literature on asymptotically de Sitter spaces. Among others, Anderson and Chruściel studied the geometry of asymptotically de Sitter spaces [Anderson 2004; 2005; Anderson and Chruściel 2005], while in [Vasy 2010b] the asymptotics of solutions of the Klein-Gordon equation were obtained, and in [Baskin 2010] the forward fundamental solution was constructed as a Fourier integral operator. It should be pointed out that the de Sitter-Schwarzschild metric in fact has many similar features with asymptotically de Sitter spaces (in an appropriate sense, it simply has two de Sitter-like ends). A weaker version of the asymptotics in this case is contained in the works of Dafermos and Rodnianski [2005; 2009; 2007] (they also study a nonlinear problem), and local energy decay was studied by Bony and Häfner [2008], in part based on the stationary resonance analysis of Sá Barreto and Zworski [1997]; stronger asymptotics (exponential decay to constants) was shown in a series of papers with Antônio Sá Barreto, Richard Melrose and the author [Melrose et al. 2011; 2008]. 
For the universal cover of AdS space itself, the Klein-Gordon equation was studied by Breitenlohner and Freedman [1982a; 1982b], who showed its solvability for $\lambda<(n-1)^{2} / 4, n=4$, and uniqueness for $\lambda<5 / 4$, in our normalization. Analogues of these results were extended to the Dirac equation by Bachelot [2008]; and on exact AdS space there is an explicit solution due to Yagdjian and Galstian [2009]. Finally, for a class of perturbations of the universal cover of AdS, which still possess a suitable Killing vector field, Holzegel [2010] showed well-posedness for $\lambda<(n-1)^{2} / 4$ by imposing a boundary condition; see [Holzegel 2010, Definition 3.1]. He also obtained certain estimates on the derivatives of the solution, as well as pointwise bounds.

Below we consider solutions of $P u=0$, or indeed $P u=f$ with $f$ given. Before describing our results, first we recall a formulation of the conformal problem, namely $\hat{g}=x^{2} g$, so $\hat{g}$ is Lorentzian smooth on $X$, and $Y$ is timelike - at the end of the introduction we give a full summary of basic results in the "compact" and "conformally compact" Riemannian and Lorentzian settings, with spacelike as well as timelike boundaries in the latter case. Let

$$
\hat{P}=\square_{\hat{g}} ;
$$

adding $\lambda$ to the operator makes no difference in this case (unlike for $P$ ). Suppose that $\mathscr{Y}$ is a spacelike hypersurface in $X$ intersecting $Y$ (automatically transversally). Then the Cauchy problem for the Dirichlet boundary condition,

$$
\hat{P} u=f,\left.\quad u\right|_{Y}=0,\left.\quad u\right|_{\mathscr{S}}=\psi_{0},\left.\quad V u\right|_{\mathscr{S}}=\psi_{1},
$$

with $f, \psi_{0}, \psi_{1}$ given, $V$ a vector field transversal to $\mathscr{Y}$, is locally well-posed (in appropriate function spaces) near $\mathscr{S}$. Moreover, under a global condition on the generalized broken bicharacteristic (or GBB) flow and $\mathscr{Y}$, which we recall below in Definition 1.1, the equation is globally well-posed.

Namely, the global geometric assumption is that

there exists $t \in \mathscr{C}^{\infty}(X)$ such that for every GBB $\gamma$, the map $t \circ \rho \circ \gamma: \mathbb{R} \rightarrow \mathbb{R}$ is either strictly increasing or strictly decreasing and has range $\mathbb{R}$,

where $\rho: T^{*} X \rightarrow X$ is the bundle projection. In the formulation above of the problem, we would assume that $\mathscr{Y}$ is a level set, $t=t_{0}$; note that locally this is always true in view of the Lorentzian nature of the metric and the conditions on $Y$ and $\mathscr{Y}$. As is often the case in the presence of boundaries - see for example [Hörmander 1985, Theorem 24.1.1] and the subsequent remark - it is convenient to consider the special case of the Cauchy problem with vanishing initial data and $f$ supported to one side of $\mathscr{Y}$, say in $t \geq t_{0}$; one can phrase this as solving

$$
\hat{P} u=f,\left.\quad u\right|_{Y}=0, \quad \operatorname{supp} u \subset\left\{t \geq t_{0}\right\} .
$$

This forward Cauchy problem is globally well-posed for $f \in L_{\mathrm{loc}}^{2}(X)$ and $u \in \dot{H}_{\mathrm{loc}}^{1}(X)$, and the analogous statement also holds for the backward Cauchy problem. Here we use Hörmander's notation $\dot{H}^{1}(X)[1985$, Appendix B] to avoid confusion with the "zero Sobolev spaces" $H_{0}^{s}(X)$, which we recall momentarily. In addition, (without any global assumptions) singularities of solutions, as measured by the b-wave front set, $\mathrm{WF}_{\mathrm{b}}$, relative to either $L_{\mathrm{loc}}^{2}(X)$ or $\dot{H}_{\mathrm{loc}}^{1}(X)$, propagate along GBB as was shown by Melrose, 
Sjöstrand and Taylor [Melrose and Sjöstrand 1978; 1982; Taylor 1976; Melrose and Taylor 1985]; see also [Sjöstrand 1980] in the analytic setting. Here recall that in $X^{\circ}$, bicharacteristics are integral curves of the Hamilton vector field $\mathrm{H}_{p}\left(\right.$ on $T^{*} X^{\circ} \backslash o$ ) of the principal symbol $\hat{p}=\sigma_{2}(\hat{P})$ inside the characteristic set,

$$
\Sigma=\hat{p}^{-1}(\{0\})
$$

We also recall that the notion of a $\mathscr{C}^{\infty}$ and an analytic GBB is somewhat different due to the behavior at diffractive points, with the analytic definition being more permissive (that is, weaker). Throughout this paper we use the analytic definition, which we now recall.

First, we need the notion of the compressed characteristic set $\dot{\Sigma}$ of $\hat{P}$. This can be obtained by replacing $T_{Y}^{*} X$ in $T^{*} X$ by its quotient $T_{Y}^{*} X / N^{*} Y$, where $N^{*} Y$ is the conormal bundle of $Y$ in $X$. One denotes then by $\dot{\Sigma}$ the image $\hat{\pi}(\Sigma)$ of $\Sigma$ in this quotient. One can give a topology to $\dot{\Sigma}$, making a set $O$ open if and only if $\hat{\pi}^{-1}(O)$ is open in $\Sigma$. This notion of the compressed characteristic set is rather intuitive, since working with the quotient encodes the law of reflection: Points with the same tangential but different normal momentum at $Y$ are identified, which, when combined with the conservation of kinetic energy (that is, working on the characteristic set) gives the standard law of reflection. However, it is very useful to introduce another (equivalent) definition already at this point since it arises from structures that we also need.

The alternative point of view (which is what one needs in the proofs) is that the analysis of solutions of the wave equation takes place on the b-cotangent bundle, ${ }^{b} T^{*} X$ (' $b$ ' stands for boundary), introduced by Melrose. See [Melrose 1993] for a very detailed description, and [Vasy 2008c] for a concise discussion. Invariantly one can define ${ }^{\mathrm{b}} T^{*} X$ as follows. First, let $\mathscr{V}_{\mathrm{b}}(X)$ be the set of all $\mathscr{C}^{\infty}$ vector fields on $X$ tangent to the boundary. If $\left(x, y_{1}, \ldots, y_{n-1}\right)$ are local coordinates on $X$, with $x$ defining $Y$, then elements of $\mathscr{V}_{\mathrm{b}}(X)$ have the form

$$
a x \partial_{x}+\sum_{j=1}^{n-1} b_{j} \partial_{y_{j}}
$$

with $a$ and $b_{j}$ smooth. It follows immediately that $\mathscr{V}_{\mathrm{b}}(X)$ is the set of all smooth sections of a vector bundle ${ }^{\mathrm{b}} T X$, and $x, y_{j}, a, b_{j}$ for $j=1, \ldots, n-1$ give local coordinates in terms of (1-2). Then ${ }^{\mathrm{b}} T^{*} X$ is defined as the dual bundle of ${ }^{\mathrm{b}} T X$. Thus, points in the b-cotangent bundle, ${ }^{\mathrm{b}} T^{*} X$, of $X$ are of the form

$$
\underline{\xi} \frac{d x}{x}+\sum_{j=1}^{n-1} \underline{\zeta}_{j} d y_{j}
$$

so $(x, y, \underline{\xi}, \underline{\zeta})$ give coordinates on ${ }^{\mathrm{b}} T^{*} X$. There is a natural map $\pi: T^{*} X \rightarrow^{\mathrm{b}} T^{*} X$ induced by the corresponding map between sections,

$$
\xi d x+\sum_{j=1}^{n-1} \zeta_{j} d y_{j}=(x \xi) \frac{d x}{x}+\sum_{j=1}^{n-1} \zeta_{j} d y_{j}
$$


Thus

$$
\pi(x, y, \xi, \zeta)=(x, y, x \xi, \zeta)
$$

that is, $\underline{\xi}=x \xi$ and $\underline{\xi}=\zeta$. Over the interior of $X$ we can identify $T_{X^{\circ}}^{*} X$ with ${ }^{\mathrm{b}} T_{X^{\circ}}^{*} X$, but this identification $\pi$ becomes singular (no longer a diffeomorphism) at $Y$. We denote the image of $\Sigma$ under $\pi$ by

$$
\dot{\Sigma}=\pi(\Sigma),
$$

called the compressed characteristic set. Thus, $\dot{\Sigma}$ is a subset of the vector bundle ${ }^{\mathrm{b}} T^{*} X$, and hence is equipped with a topology that is equivalent to the one define by the quotient; see [Vasy 2008c, Section 5]. The definition of analytic GBB is then as follows:

Definition 1.1. Generalized broken bicharacteristics, or GBB, are continuous maps $\gamma: I \rightarrow \dot{\Sigma}$, where $I$ is an interval, satisfying that for all $f \in \mathscr{C}^{\infty}\left({ }^{\mathrm{b}} T^{*} X\right)$ real valued,

$$
\liminf _{s \rightarrow s_{0}} \frac{(f \circ \gamma)(s)-(f \circ \gamma)\left(s_{0}\right)}{s-s_{0}} \geq \inf \left\{\mathrm{H}_{p}\left(\pi^{*} f\right)(q): q \in \pi^{-1}\left(\gamma\left(s_{0}\right)\right) \cap \Sigma\right\} .
$$

Since the map $p \mapsto \mathrm{H}_{p}$ is a derivation, $\mathrm{H}_{a p}=a \mathrm{H}_{p}$ at $\Sigma$, so bicharacteristics are merely reparametrized if $p$ is replaced by a conformal multiple. In particular, if $P$ is the Klein-Gordon operator $\square_{g}+\lambda$ for an asymptotically AdS-metric $g$, the bicharacteristics over $X^{\circ}$ are, up to reparametrization, those of $\hat{g}$. We make this into our definition of GBB.

Definition 1.2. The compressed characteristic set $\dot{\Sigma}$ of $P$ is that of $\square_{\hat{g}}$.

Generalized broken bicharacteristics, or GBB, of $P$ are GBB in the analytic sense of the smooth Lorentzian metric $\hat{g}$.

We now give a formulation for the global problem. For this purpose we need to recall one more class of differential operators in addition to $\mathscr{V}_{\mathrm{b}}(X)$ (which is the set of $\mathscr{C}^{\infty}$ vector fields tangent to the boundary). Namely, we denote the set of $\mathscr{C}^{\infty}$ vector fields vanishing at the boundary by $\mathscr{V}_{0}(X)$. In local coordinates $(x, y)$, these have the form

$$
a x \partial_{x}+\sum_{j=1}^{n} b_{j}\left(x \partial_{y_{j}}\right), \quad \text { with } a, b_{j} \in \mathscr{C}^{\infty}(X)
$$

see (1-2). Again, $\mathscr{V}_{0}(X)$ is the set of all $\mathscr{C}^{\infty}$ sections of a vector bundle ${ }^{0} T X$, which over $X^{\circ}$ can be naturally identified with $T_{X^{\circ}} X$; see [Mazzeo and Melrose 1987] for a detailed discussion of 0-geometry and analysis and [Vasy 2010b] for a summary. We then let $\operatorname{Diff}_{\mathrm{b}}(X)$ and $\operatorname{Diff}_{0}(X)$ be the set of differential operators generated by $\mathscr{V}_{\mathrm{b}}(X)$ and $\mathscr{V}_{0}(X)$, respectively, that is, they are locally finite sums of products of these vector fields with $\mathscr{C}^{\infty}(X)$-coefficients. In particular,

$$
P=\square_{g}+\lambda \in \operatorname{Diff}_{0}^{2}(X),
$$

which explains the relevance of $\operatorname{Diff}_{0}(X)$. This can be seen easily from $g$ being in fact a nondegenerate smooth symmetric bilinear form on ${ }^{0} T X$; the conformal factor $x^{-2}$ compensates for the vanishing factors of $x$ in (1-4), so in fact this is exactly the same statement as $\hat{g}$ being Lorentzian on $T X$. 
Let $H_{0}^{k}(X)$ denote the zero-Sobolev space relative to

$$
L^{2}(X)=L_{0}^{2}(X)=L^{2}(X, d g)=L^{2}\left(X, x^{-n} d \hat{g}\right) ;
$$

so if $k \geq 0$ is an integer then

$$
u \in H_{0}^{k}(X) \quad \text { if and only if } \quad L u \in L^{2}(X) \text { for all } L \in \operatorname{Diff}_{0}^{k}(X) ;
$$

negative values of $k$ give Sobolev spaces by dualization. For our problem, we need a space of "very nice" functions corresponding to $\operatorname{Diff}_{\mathrm{b}}(X)$. We obtain this by replacing $\mathscr{C}^{\infty}(X)$ with the space of conormal functions to the boundary relative to a fixed space of functions, in this case $H_{0}^{k}(X)$, that is, functions $v \in H_{0, \text { loc }}^{k}(X)$ such that $Q v \in H_{0, \text { loc }}^{k}(X)$ for every $Q \in \operatorname{Diff}_{\mathrm{b}}(X)$ (of any order). The finite order regularity version of this is $H_{0, \mathrm{~b}}^{k, m}(X)$, which is given for $m \geq 0$ integer by

$$
u \in H_{0, \mathrm{~b}}^{k, m}(X) \quad \text { if and only if } \quad u \in H_{0}^{k}(X) \text { and } Q u \in H_{0}^{k}(X) \text { for all } Q \in \operatorname{Diff}_{\mathrm{b}}^{m}(X),
$$

while for $m<0$ integer, $u \in H_{0, \mathrm{~b}}^{k, m}(X)$ if $u=\sum Q_{j} u_{j}, u_{j} \in H_{0, \mathrm{~b}}^{k, 0}(X)$, and $Q_{j} \in \operatorname{Diff}_{\mathrm{b}}^{m}(X)$. Thus, $H_{0, \mathrm{~b}}^{-k,-m}(X)$ is the dual space of $H_{0, \mathrm{~b}}^{k, m}(X)$, relative to $L_{0}^{2}(X)$. Note that in $X^{\circ}$, there is no distinction between $\mathscr{V}_{\mathrm{b}}(X), \mathscr{V}_{0}(X)$, or indeed simply $\mathscr{V}(X)$ (smooth vector fields on $X$ ), so over compact subsets $K$ of $X^{\circ}, H_{0, \mathrm{~b}}^{k, m}(X)$ is the same as $H^{k+m}(K)$. On the other hand, at $Y=\partial X, H_{0, \mathrm{~b}}^{k, m}(X)$ distinguishes precisely between regularity relative to $\mathscr{V}_{0}(X)$ and $\mathscr{V}_{\mathrm{b}}(X)$.

Although the finite speed of propagation means that the wave equation has a local character in $X$, and thus compactness of the slices $t=t_{0}$ is immaterial, it is convenient to assume that

$$
\text { the map } t: X \rightarrow \mathbb{R} \text { is proper. }
$$

Even as stated, the propagation of singularities results (which form the heart of the paper) do not assume this, and the assumption is made elsewhere merely to make the formulation and proof of the energy estimates and existence slightly simpler, in that one does not have to localize in spatial slices this way.

Suppose $\lambda<(n-1)^{2} / 4$. Suppose

$$
f \in H_{0, \mathrm{~b}, \mathrm{loc}}^{-1,1}(X) \quad \text { and } \quad \operatorname{supp} f \subset\left\{t \geq t_{0}\right\} .
$$

We want to find $u \in H_{0, \text { loc }}^{1}(X)$ such that

$$
P u=f \quad \text { and } \quad \operatorname{supp} u \subset\left\{t \geq t_{0}\right\} .
$$

We show that this is locally well-posed near $\mathscr{S}$. Moreover, under the previous global assumption on GBB, this problem is globally well-posed:

Theorem 1.3 (see Theorem 4.16). Assume that (TF) and (PT) hold. Suppose $\lambda<(n-1)^{2} / 4$. The forward Dirichlet problem (1-6) has a unique global solution $u \in H_{0,10 c}^{1}(X)$, and for all compact $K \subset X$ there exists a compact $K^{\prime} \subset X$ and a constant $C>0$ such that for all $f$ as in (1-5), the solution $u$ satisfies

$$
\|u\|_{H_{0}^{1}(K)} \leq C\|f\|_{H_{0, \mathrm{~b}}^{-1,1}\left(K^{\prime}\right)} .
$$


Remark 1.4. In fact, one can be quite explicit about $K^{\prime}$ in view of (PT), since $\left.u\right|_{t \in\left[t_{0}, t_{1}\right]}$ can be estimated by $\left.f\right|_{t \in I}$, with $I$ open and containing $\left[t_{0}, t_{1}\right]$.

We also prove microlocal elliptic regularity and describe the propagation of singularities of solutions, as measured by $\mathrm{WF}_{\mathrm{b}}$ relative to $H_{0, \mathrm{loc}}^{1}(X)$. We define this notion in Definition 5.9 and discuss it there in more detail. However, we recall the definition of the standard wave front set WF on manifolds without boundary $X$ that immediately generalizes to the b-wave front set $\mathrm{WF}_{\mathrm{b}}$. Thus, one says that $q \in T^{*} X \backslash o$ is not in the wave front set of a distribution $u$ if there exists $A \in \Psi^{0}(X)$ such $\sigma_{0}(A)(q)$ is invertible and $Q A u \in L^{2}(X)$ for all $Q \in \operatorname{Diff}(X)$ - this is equivalent to $A u \in \mathscr{C}^{\infty}(X)$ by the Sobolev embedding theorem. Here $L^{2}(X)$ can be replaced by $H^{m}(X)$ instead, with $m$ arbitrary. Moreover, $\mathrm{WF}^{m}$ can also be defined analogously, by requiring $A u \in L^{2}(X)$ for $A \in \Psi^{m}(X)$ elliptic at $q$. Thus, $q \notin \mathrm{WF}(u)$ means that $u$ is 'microlocally $\mathscr{C}^{\infty}$ at $q$ ', while $q \notin \mathrm{WF}^{m}(u)$ means that $u$ is 'microlocally $H^{m}$ at $q$ '.

In order to microlocalize $H_{0, \mathrm{~b}}^{k, m}(X)$, we need pseudodifferential operators, here extending $\operatorname{Diff}{ }_{\mathrm{b}}(X)$ (as that is how we measure regularity). These are the b-pseudodifferential operators $A \in \Psi_{\mathrm{b}}^{m}(X)$ introduced by Melrose; their principal symbol $\sigma_{\mathrm{b}, m}(A)$ is a homogeneous degree $m$ function on ${ }^{\mathrm{b}} T^{*} X \backslash o$. See again [Melrose 1993; Vasy 2008c]. Then we say that $q \in{ }^{\mathrm{b}} T^{*} X \backslash o$ is not in $\mathrm{WF}_{\mathrm{b}}^{k, \infty}(u)$ if there exists $A \in \Psi_{\mathrm{b}}^{0}(X)$ with $\sigma_{\mathrm{b}, 0}(A)(q)$ invertible and such that $A u$ is $H_{0}^{k}$-conormal to the boundary. One also defines $\mathrm{WF}_{\mathrm{b}}^{k, m}(u)$ : We say $q \notin \mathrm{WF}_{\mathrm{b}}^{m}(u)$ if there exists $A \in \Psi_{\mathrm{b}}^{m}(X)$ with $\sigma_{\mathrm{b}, 0}(A)(q)$ invertible and such that $A u \in H_{0, \mathrm{loc}}^{k}(X)$. One can also extend these definitions to $m<0$.

With this definition we have the following theorem:

Theorem 1.5 (see Proposition 7.7 and Theorem 8.8). Suppose that $P=\square_{g}+\lambda$, where $\lambda<(n-1)^{2} / 4$. Let $m \in \mathbb{R}$ or $m=\infty$. Suppose $u \in H_{0, \mathrm{~b}, \text { loc }}^{1, k}(X)$ for some $k \in \mathbb{R}$. Then

$$
\mathrm{WF}_{\mathrm{b}}^{1, m}(u) \backslash \dot{\Sigma} \subset \mathrm{WF}_{\mathrm{b}}^{-1, m}(P u) .
$$

Moreover,

$$
\left(\mathrm{WF}_{\mathrm{b}}^{1, m}(u) \cap \dot{\Sigma}\right) \backslash \mathrm{WF}_{\mathrm{b}}^{-1, m+1}(P u)
$$

is a union of maximally extended generalized broken bicharacteristics of the conformal metric $\hat{g}$ in

$$
\dot{\Sigma} \backslash \mathrm{WF}_{\mathrm{b}}^{-1, m+1}(P u) .
$$

In particular, if $P u=0$, then $\mathrm{WF}_{\mathrm{b}}^{1, \infty}(u) \subset \dot{\Sigma}$ is a union of maximally extended generalized broken bicharacteristics of $\hat{g}$.

As a consequence of this theorem, we get a more general, and precise, well-posedness result:

Theorem 1.6 (see Theorem 8.12). Assume that $(T F)$ and $(P T)$ hold. Suppose that $P=\square_{g}+\lambda$, where $\lambda<(n-1)^{2} / 4$. Let $m \in \mathbb{R}$ and suppose $m^{\prime} \leq m$. Suppose $f \in H_{0, \mathrm{~b}, \mathrm{loc}}^{-1, m+1}(X)$. Then (1-6) has a unique solution in $H_{0, \mathrm{~b}, \mathrm{loc}}^{1, m^{\prime}}(X)$, which in fact lies in $H_{0, \mathrm{~b}, \mathrm{loc}}^{1, m}(X)$, and for all compact $K \subset X$ there exists a compact $K^{\prime} \subset X$ and a constant $C>0$ such that

$$
\|u\|_{H_{0}^{1, m}(K)} \leq C\|f\|_{H_{0, \mathrm{~b}}^{-1, m+1}\left(K^{\prime}\right)} .
$$


While we prove this result using the relatively sophisticated technique of propagation of singularities, it could also be derived without full microlocalization, that is, without localizing the propagation of energy in phase space.

We also generalize propagation of singularities to the case $\operatorname{Im} \lambda \neq 0$ ( $\operatorname{Re} \lambda$ arbitrary), in which case we prove one-sided propagation depending on the sign of $\operatorname{Im} \lambda$. Namely, if $\operatorname{Im} \lambda>0$ respectively $\operatorname{Im} \lambda<0$, then

$$
\left(\mathrm{WF}_{\mathrm{b}}^{1, m}(u) \cap \dot{\Sigma}\right) \backslash \mathrm{WF}_{\mathrm{b}}^{-1, m+1}(P u)
$$

is a union of maximally forward respectively maximally backward extended generalized broken bicharacteristics of the conformal metric $\hat{g}$. There is no difference between the case $\operatorname{Im} \lambda=0$ and $\operatorname{Re} \lambda<(n-1)^{2} / 4$, respectively $\operatorname{Im} \lambda \neq 0$, at the elliptic set, that is, the statement

$$
\mathrm{WF}_{\mathrm{b}}^{1, m}(u) \backslash \dot{\Sigma} \subset \mathrm{WF}_{\mathrm{b}}^{-1, m}(P u) .
$$

holds even if $\operatorname{Im} \lambda \neq 0$. We refer to Proposition 7.7 and Theorem 8.9 for details.

These results indicate already that for $\operatorname{Im} \lambda \neq 0$ there are many interesting questions to answer, and in particular that one cannot think of $\lambda$ as 'small'; this will be the focus of future work.

In particular, if $f$ is conormal relative to $H_{0}^{1}(X)$ then $\mathrm{WF}_{\mathrm{b}}^{1, \infty}(u)=\varnothing$. Let $\sqrt{\cdot}$ denote the branch square root function on $\mathbb{C} \backslash(-\infty, 0]$ chosen so that takes positive values on $(0, \infty)$. The simplest conormal functions are those in $\mathscr{C}^{\infty}(X)$ that vanish to infinite order (that is, with all derivatives) at the boundary; the set of these is denoted by $\dot{\mathscr{C}}^{\infty}(X)$. If we assume $f \in \dot{\mathscr{C}}^{\infty}(X)$ then

$$
u=x^{s_{+}(\lambda)} v, \quad v \in \mathscr{C}^{\infty}(X), \quad s_{+}(\lambda)=\frac{1}{2}(n-1)+\sqrt{\frac{1}{4}(n-1)^{2}-\lambda},
$$

as we show in Proposition 8.10. Since the indicial roots of $\square_{g}+\lambda$ are

$$
s_{ \pm}(\lambda)=\frac{1}{2}(n-1) \pm \sqrt{\frac{1}{4}(n-1)^{2}-\lambda}
$$

this explains the interpretation of this problem as a "Dirichlet problem", much like it was done in the Riemannian conformally compact case by Mazzeo and Melrose [1987]: Asymptotics $x^{s_{-}(\lambda)} v_{-}$, with $v_{-} \in \mathscr{C}^{\infty}(X)$, corresponding to the growing indicial root $s_{-}(\lambda)$ is ruled out.

For $\lambda<(n-1)^{2} / 4$, one can then easily solve the problem with inhomogeneous "Dirichlet" boundary condition, that is, given $v_{0} \in \mathscr{C}^{\infty}(Y)$ and $f \in \dot{\mathscr{C}}^{\infty}(X)$, both supported in $\left\{t \geq t_{0}\right\}$,

$$
P u=f,\left.\quad u\right|_{t<t_{0}}=0, \quad u=x^{s_{-}(\lambda)} v_{-}+x^{s_{+}(\lambda)} v_{+}, \quad v_{ \pm} \in \mathscr{C}^{\infty}(X),\left.\quad v_{-}\right|_{Y}=v_{0}
$$

if $s_{+}(\lambda)-s_{-}(\lambda)=2 \sqrt{(n-1)^{2} / 4-\lambda}$ is not an integer. If $s_{+}(\lambda)-s_{-}(\lambda)$ is an integer, the same conclusion holds if we replace $v_{-} \in \mathscr{C}^{\infty}(X)$ by $v_{-} \in \mathscr{C}^{\infty}(X)+x^{s_{+}(\lambda)-s_{-}(\lambda)} \log x \mathscr{C}^{\infty}(X)$; see Theorem 8.11.

The operator $\left.\left.v_{-}\right|_{Y} \rightarrow v_{+}\right|_{Y}$ is the analogue of the Dirichlet-to-Neumann map, or the scattering operator. In the De Sitter setting the setup is somewhat different as both pieces of scattering data are specified either at past or future infinity; see [Vasy 2010b]. Nonetheless, one expects that the result of [ibid., Section 7], that the scattering operator is a Fourier integral operator associated to the GBB relation, can be extended to the present setting, at least if the boundary is totally geodesic with respect to the conformal metric $\hat{g}$ 
and the metric is even with respect to the boundary in an appropriate sense. Indeed, in an ongoing project, Baskin and the author are extending Baskin's construction [2010] of the forward fundamental solution on asymptotically De Sitter spaces to the even totally geodesic asymptotically AdS setting. In addition, it is interesting to ask what the "best" problem to pose is when $\operatorname{Im} \lambda \neq 0$; the results of this paper suggest that the global problem (rather than local, Cauchy data versions) is the best behaved. One virtue of the parametrix construction is that we expect to be able answer Lorentzian analogues of questions related to [Mazzeo and Melrose 1987], which would bring the Lorentzian world of AdS spaces significantly closer (in terms of results) to the Riemannian world of conformally compact spaces. We singled out the totally geodesic condition and evenness since they hold on actual AdS space, which we now discuss.

We now recall the structure of the actual AdS space to justify our terminology. Consider $\mathbb{R}^{n+1}$ with the pseudo-Riemannian metric of signature $(2, n-1)$ given by

$$
-d z_{1}^{2}-\cdots-d z_{n-1}^{2}+d z_{n}^{2}+d z_{n+1}^{2}
$$

with $\left(z_{1}, \ldots, z_{n+1}\right)$ denoting coordinates on $\mathbb{R}^{n+1}$, and the hyperboloid

$$
z_{1}^{2}+\cdots+z_{n-1}^{2}-z_{n}^{2}-z_{n+1}^{2}=-1
$$

inside it. Note that $z_{n}^{2}+z_{n+1}^{2} \geq 1$ on the hyperboloid, so we can (diffeomorphically) introduce polar coordinates in these two variables, that is, we let $\left(z_{n}, z_{n+1}\right)=R \theta$, with $R \geq 1$ and $\theta \in \mathbb{S}^{1}$. Then the hyperboloid is of the form

$$
z_{1}^{2}+\cdots+z_{n-1}^{2}-R^{2}=-1
$$

inside $\mathbb{R}^{n-1} \times(0, \infty)_{R} \times \mathbb{S}_{\theta}^{1}$. Since $d z_{j}$ for $j=1, \ldots, n-1, d \theta$ and $d\left(z_{1}^{2}+\cdots+z_{n-1}^{2}-R^{2}\right)$ are linearly independent at the hyperboloid,

$$
z_{1}, \ldots, z_{n-1}, \theta
$$

give local coordinates on it, and indeed these are global in the sense that the hyperboloid $X^{\circ}$ is identified with $\mathbb{R}^{n-1} \times \mathbb{S}^{1}$ via these. A straightforward calculation shows that the metric on $\mathbb{R}^{n+1}$ restricts to give a Lorentzian metric $g$ on the hyperboloid. Indeed, away from $\{0\} \times \mathbb{S}^{1}$, we obtain a convenient form of the metric by using polar coordinates $(r, \omega)$ in $\mathbb{R}^{n-1}$, so $R^{2}=r^{2}+1$ :

$$
g=-(d r)^{2}-r^{2} d \omega^{2}+(d R)^{2}+R^{2} d \theta^{2}=-\left(1+r^{2}\right)^{-1} d r^{2}-r^{2} d \omega^{2}+\left(1+r^{2}\right) d \theta^{2},
$$

where $d \omega^{2}$ is the standard round metric; a similar description is easily obtained near $\{0\} \times \mathbb{S}^{1}$ by using the standard Euclidean variables.

We can compactify the hyperboloid by compactifying $\mathbb{R}^{n-1}$ to a ball $\overline{\mathbb{B}^{n-1}}$ via inverse polar coordinates $(x, \omega)$, where $x=r^{-1}$,

$$
\left(z_{1}, \ldots, z_{n-1}\right)=x^{-1} \omega, \quad 0<x<\infty, \quad \omega \in \mathbb{S}^{n-2} .
$$

Thus, the interior of $\overline{\mathbb{B}^{n-1}}$ is identified with $\mathbb{R}^{n-1}$, and the boundary $\mathbb{S}^{n-2}$ of $\overline{\mathbb{B}^{n-1}}$ is added at $x=0$ to compactify $\mathbb{R}^{n-1}$. We let

$$
X=\overline{\mathbb{B}^{n-1}} \times \mathbb{S}^{1}
$$


be this compactification of $X^{\circ}$; a collar neighborhood of $\partial X$ is identified with

$$
[0,1)_{x} \times \mathbb{S}_{\omega}^{n-2} \times \mathbb{S}_{\theta}^{1} .
$$

In this collar neighborhood, the Lorentzian metric takes the form

$$
g=\frac{1}{x^{2}}\left(-\left(1+x^{2}\right)^{-1} d x^{2}-d \omega^{2}+\left(1+x^{2}\right) d \theta^{2}\right),
$$

which is of the desired form, and the conformal metric is

$$
\hat{g}=-\left(1+x^{2}\right)^{-1} d x^{2}-d \omega^{2}+\left(1+x^{2}\right) d \theta^{2}
$$

with respect to which the boundary $\{x=0\}$ is indeed timelike. Note that the induced metric on the boundary is $-d \omega^{2}+d \theta^{2}$ up to a conformal multiple.

As already remarked, $\hat{g}$ has the special feature that $Y$ is totally geodesic, unlike for example the case of $\mathbb{B}^{n-1} \times \mathbb{S}^{1}$ equipped with a product Lorentzian metric, with $\mathbb{B}^{n-1}$ carrying the standard Euclidean metric.

For global results, it is useful to work on the universal cover $\tilde{X}=\overline{\mathbb{B}^{n-1}} \times \mathbb{R}_{t}$ of $X$, where $\mathbb{R}_{t}$ is the universal cover of $\mathbb{S}_{\theta}^{1}$; we use $t$ to emphasize the timelike nature of this coordinate. The local geometry is unchanged, but now $t$ provides a global parameter along generalized broken bicharacteristics, and satisfies the assumptions (TF) and (PT) for our theorems.

We use this opportunity to summarize the results, already referred to earlier, for analysis on conformally compact Riemannian or Lorentzian spaces, including a comparison with the conformally related problem, that is, for $\Delta_{\hat{g}}$ or $\square_{\hat{g}}$. We assume Dirichlet boundary conditions (DBC) when relevant for the sake of definiteness, and global hyperbolicity for the hyperbolic equations, and do not state the function spaces or optimal forms of regularity results.

(i) Riemannian: $\left(\Delta_{\hat{g}}-\lambda\right) u=f$ with DBC is well-posed for $\lambda \in \mathbb{C} \backslash[0, \infty)$; moreover, if $f \in \dot{\mathscr{C}}^{\infty}(X)$, then $u \in \mathscr{C}^{\infty}(X)$. (This also works outside a discrete set of poles $\lambda$ in $[0, \infty)$.)

(ii) Lorentzian, $\partial X=Y_{+} \cup Y_{-}$is spacelike, $f$ is supported in $t \geq t_{0}$, and $\lambda \in \mathbb{C}$ : $\left(\square_{\hat{g}}-\lambda\right) u=f$, for $u$ supported in $t \geq t_{0}$, is well-posed. If $f \in \dot{\mathscr{C}}^{\infty}(X)$, the solution is $\mathscr{C}^{\infty}$ up to $Y_{ \pm}$.

(iii) Lorentzian, $\partial X$ is timelike, $f$ is supported in $t \geq t_{0}$, and $\lambda \in \mathbb{C}$ : $\left(\square_{\hat{g}}-\lambda\right) u=f$, with DBC at $Y$ and $u$ supported in $t \geq t_{0}$, is well-posed. If $f \in \dot{\mathscr{C}}^{\infty}(X)$, the solution is $\mathscr{C}^{\infty}$ up to $Y_{ \pm}$.

We now go through the original problems. Let $s_{ \pm}(\lambda)$ be as in (1-7).

(i) Asymptotically hyperbolic, $\lambda \in \mathbb{C} \backslash[0,+\infty)$ : There is a unique solution of $\left(\Delta_{g}-\lambda\right) u=f$, with $f \in \dot{\mathscr{C}}^{\infty}(X)$, such that $u=x^{s_{+}(\lambda)} v, v \in \mathscr{C}^{\infty}(X)$. (Analogue of DBC [Mazzeo and Melrose 1987].) (Indeed, $u=\left(\Delta_{g}-\lambda\right)^{-1} f$, and this can be extended to $\lambda \in[0,+\infty)$, apart from finitely many poles in $\left[0,(n-1)^{2} / 4\right]$, and analytically continued further.)

(ii) Asymptotically de Sitter, $\lambda \in \mathbb{C}$ : For $f$ supported in $t \geq t_{0}$, there is a unique solution of $\left(\square_{g}-\lambda\right) u=f$ supported in $t \geq t_{0}$. Moreover, for $f \in \dot{\mathscr{C}}^{\infty}(X)$,

$$
u=x^{s_{+}(\lambda)} v_{+}+x^{s_{-}(\lambda)} v_{-}, \quad v_{ \pm} \in \mathscr{C}^{\infty}(X), \quad \text { and }\left.v_{ \pm}\right|_{Y_{-}} \text {is specified, }
$$



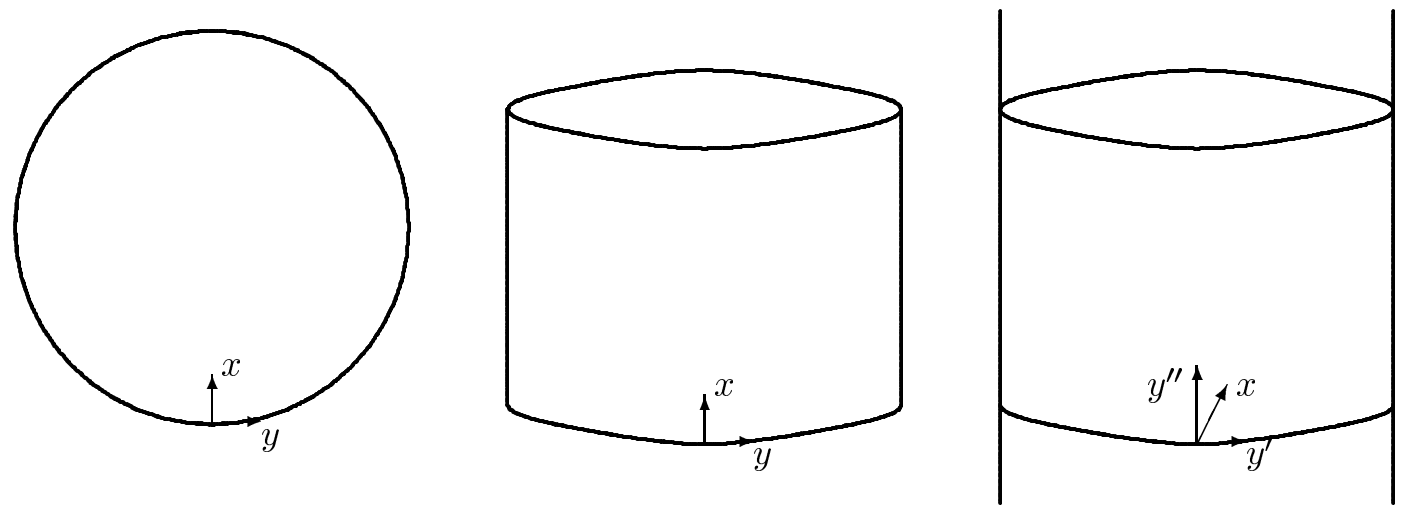

Figure 1. On the left, a Riemannian example, $\overline{\mathbb{B}^{2}}$. In the middle, an example of spacelike boundary, $[0,1]_{x} \times \mathbb{S}_{y}^{1}$ with $x$ timelike. On the right, the case of timelike boundary, $\overline{\mathbb{B}_{x, y^{\prime}}^{2}} \times \mathbb{R}_{y^{\prime \prime}}$, with $y^{\prime \prime}$ timelike.

provided that $s_{+}(\lambda)-s_{-}(\lambda) \notin \mathbb{Z}$. (See [Vasy 2010b].)

(iii) Asymptotically anti de Sitter, $\lambda \in \mathbb{R} \backslash\left[(n-1)^{2} / 4,+\infty\right)$ : For $f \in \dot{\mathscr{C}}^{\infty}(X)$ supported in $t \geq t_{0}$, there is a unique solution of $\left(\square_{g}-\lambda\right) u=f$ such that $u=x^{s_{+}(\lambda)} v, v \in \mathscr{C}^{\infty}(X)$ and $\operatorname{supp} u \subset\left\{t \geq t_{0}\right\}$.

The structure of this paper is as follows. In Section 2 we prove a Poincaré inequality that we use to allow the sharp range $\lambda<(n-1)^{2} / 4$ for $\lambda$ real. Then in Section 3 we recall the structure of energy estimates on manifolds without boundary as these are then adapted to our "zero geometry" in Section 4. In Section 5 we introduce microlocal tools to study operators such as $P$, namely the zero-differentialb-pseudodifferential calculus, $\operatorname{Diff}_{0} \Psi_{\mathrm{b}}(X)$. In Section 6 the structure of GBB is recalled. In Section 7 we study the Dirichlet form and prove microlocal elliptic regularity. Finally, in Section 8, we prove the propagation of singularities for $P$.

\section{Poincaré inequality}

Let $h$ be a conformally compact Riemannian metric, that is, a positive definite inner product on ${ }^{0} T X$ and hence by duality on ${ }^{0} T^{*} X$; we denote the latter by $H$. We denote the corresponding space of $L^{2}$ sections of ${ }^{0} T^{*} X$ by $L^{2}\left(X ;{ }^{0} T^{*} X\right)=L_{0}^{2}\left(X ;{ }^{0} T^{*} X\right)$. While the inner product on $L^{2}\left(X ;{ }^{0} T^{*} X\right)$ depends on the choice of $h$, the corresponding norms are independent of $h$, at least over compact subsets $K$ of $X$. We first prove a Hardy-type inequality:

Lemma 2.1. Suppose $V_{0} \in \mathscr{V}(X)$ is real with $\left.V_{0} x\right|_{x=0}=1$, and let $V \in \mathscr{V}_{\mathrm{b}}(X)$ be given by $V=x V_{0}$. Given any compact subset $K$ of $X$ and $\tilde{C}<(n-1) / 2$, there exists $x_{0}>0$ such that if $u \in \dot{\mathscr{C}}^{\infty}(X)$ is supported in $K$, then for $\psi \in \mathscr{C}^{\infty}(X)$ supported in $x<x_{0}$,

$$
\tilde{C}\|\psi u\|_{L_{0}^{2}(X)} \leq\|\psi V u\|_{L_{0}^{2}(X)}
$$


Recall here that $\dot{\mathscr{C}}^{\infty}(X)$ denotes elements of $\mathscr{C}^{\infty}(X)$ that vanish at $Y=\partial X$ to infinite order, and the subscript comp on $\dot{\mathscr{C}}_{\text {comp }}^{\infty}(X)$ below indicates that in addition the support of the function under consideration is compact.

Proof. For any $V \in \mathscr{V}_{\mathrm{b}}(X)$ real, and $\chi \in \mathscr{C}_{\text {comp }}^{\infty}(X), u \in \dot{\mathscr{C}}_{\text {comp }}^{\infty}(X)$, we have, using $V^{*}=-V-\operatorname{div} V$,

$\langle(V \chi) u, u\rangle=\langle[V, \chi] u, u\rangle=\left\langle\chi u, V^{*} u\right\rangle-\langle V u, \chi u\rangle=-\langle\chi u, V u\rangle-\langle V u, \chi u\rangle-\langle\chi u,(\operatorname{div} V) u\rangle$.

Now, if $V=x V_{0}$, with $V_{0} \in \mathscr{V}(X)$ transversal to $\partial X$, and if we write $d g=x^{-n} d \hat{g}$ for $d \hat{g}$ a smooth nondegenerate density, then in local coordinates $z_{j}$ such that $d \hat{g}=J|d z|$ and $V_{0}=\sum V_{0}^{j} \partial_{j}$,

$$
\begin{aligned}
\operatorname{div} V & =x^{n} J^{-1} \sum \partial_{j}\left(x^{-n} J x V_{0}^{j}\right) \\
& =-(n-1) \sum_{j} V_{0}^{j}\left(\partial_{j} x\right)+x J^{-1} \sum \partial_{j}\left(J V_{0}^{j}\right)=-(n-1)\left(V_{0} x\right)+x \operatorname{div}_{\hat{g}} V_{0},
\end{aligned}
$$

where the subscript $\hat{g}$ in $\operatorname{div}_{\hat{g}} V_{0}$ denotes that the divergence is with respect to $\hat{g}$. Thus, assuming that $V_{0} \in \mathscr{V}(X)$ with $\left.V_{0} x\right|_{x=0}=1$, we have

$$
\operatorname{div} V=-(n-1)+x a, \quad \text { where } a \in \mathscr{C}^{\infty}(X) .
$$

Let $x_{0}^{\prime}>0$ be such that $V_{0} x>1 / 2$ in $x \leq x_{0}^{\prime}$. Thus, if $0 \leq \chi_{0} \leq 1, \chi_{0} \equiv 1$ near $0, \chi_{0}^{\prime} \leq 0, \chi_{0}$ is supported in $x \leq x_{0}^{\prime}$, and $\chi=\chi_{0} \circ x$, then

$$
V \chi=x\left(V_{0} x\right)\left(\chi_{0}^{\prime} \circ x\right) \leq 0
$$

hence $\langle(V \chi) u, u\rangle \leq 0$ and

$$
\langle\chi((n-1)+x a) u, u\rangle \leq 2\left\|\chi^{1 / 2} u\right\|\left\|\chi^{1 / 2} V u\right\| .
$$

Thus given any $\tilde{C}<(n-1) / 2$, there is $x_{0}>0$ such that for $u$ supported in $K$,

$$
\tilde{C}\left\|\chi^{1 / 2} u\right\| \leq\left\|\chi^{1 / 2} V u\right\|
$$

namely we take $x_{0}<x_{0}^{\prime} / 2$ such that $(n-1) / 2-\tilde{C}>\left(\sup _{K}|a|\right) x_{0}$, and choose $\chi_{0} \equiv 1$ on $\left[0, x_{0}\right]$ and supported in $\left[0,2 x_{0}\right)$. This completes the proof of the lemma.

The basic Poincaré estimate is this:

Proposition 2.2. Suppose $K \subset X$ compact, $K \cap \partial X \neq \varnothing, O$ is open with $K \subset O, O$ is arcwise connected to $\partial X$, and $K^{\prime}=\bar{O}$ compact. There exists $C>0$ such that for $u \in H_{0, \mathrm{loc}}^{1}(X)$, one has

$$
\|u\|_{L_{0}^{2}(K)} \leq C\|d u\|_{L_{0}^{2}\left(O ; T^{*} X\right)}
$$

where the norms are relative to the metric $h$.

Proof. It suffices to prove the estimate for $u \in \dot{\mathscr{C}}^{\infty}(X)$, for then the proposition follows by the density of $\dot{\mathscr{C}}^{\infty}(X)$ in $H_{0, \text { loc }}^{1}(X)$ and the continuity of both sides in the $H_{0, \text { loc }}^{1}(X)$ topology.

Let $V_{0}$ and $V$ be as in Lemma 2.1, and let $\phi_{0} \in \mathscr{C}_{\text {comp }}^{\infty}(Y)$ be identically 1 on a neighborhood of $K \cap Y$, supported in $O$, and let $x_{0}>0$ be as in the lemma with $K$ replaced by $K^{\prime}$. We pull back $\phi_{0}$ to a function $\phi$ defined on a neighborhood of $Y$ by the $V_{0}$ flow; thus, $V_{0} \phi=0$. By decreasing $x_{0}$ if needed, we may 
assume that $\phi$ is defined and is $\mathscr{C}^{\infty}$ in $x<x_{0}$, and supp $\phi \cap\left\{x<x_{0}\right\} \subset O$. Now, let $\psi \in \mathscr{C}^{\infty}(X)$ be identically 1 where $x<x_{0} / 2$, supported where $x<3 x_{0} / 4$, and let $\psi_{0} \in \mathscr{C}^{\infty}(X)$ be identically 1 where $x<3 x_{0} / 4$, supported in $x<x_{0}$; thus $\psi_{0} \phi \in \mathscr{C}_{\text {comp }}^{\infty}(X)$. Then, by Lemma 2.1 applied to $\psi_{0} \phi u$,

$$
\tilde{C}\|\psi \phi u\|_{L_{0}^{2}(X)}=\tilde{C}\left\|\psi \psi_{0} \phi u\right\|_{L_{0}^{2}(X)} \leq\left\|\psi V\left(\psi_{0} \phi u\right)\right\|_{L_{0}^{2}(X)}=\|\psi \phi V u\|_{L_{0}^{2}(X)} .
$$

The proposition follows by the standard Poincare estimate and arcwise connectedness of $K$ to $Y$ (hence to $x<x_{0} / 2$ ), since one can estimate $\left.u\right|_{x>x_{0} / 2}$ in $L^{2}$ in terms of $\left.d u\right|_{x>x_{0} / 2}$ in $L^{2}$ and $\left.u\right|_{x_{0} / 4<x<x_{0} / 2}$.

We can get a more precise estimate of the constants if we restrict to a neighborhood of a spacelike hypersurface $\mathscr{S}$; it is convenient to state the result under our global assumptions. Thus, (TF) and (PT) are assumed to hold from here on in this section.

Proposition 2.3. Suppose $V_{0} \in \mathscr{V}(X)$ is real with $\left.V_{0} x\right|_{x=0}=1$ and $V_{0} t \equiv 0$ near $Y$ and let $V \in \mathscr{V}_{\mathrm{b}}(X)$ be given by $V=x V_{0}$. Let I be a compact interval. Let $C<(n-1) / 2$ and $\gamma>0$. Then there exist $\epsilon>0$, $x_{0}>0$ and $C^{\prime}>0$ such that the following holds.

For $t_{0} \in I, 0<\delta<\epsilon$ and for $u \in H_{0, \mathrm{loc}}^{1}(X)$, one has

$$
\begin{array}{r}
\|u\|_{L_{0}^{2}\left(\left\{p: t(p) \in\left[t_{0}, t_{0}+\epsilon\right]\right)\right\}} \leq C^{-1}\|V u\|_{L_{0}^{2}\left(\left\{p: t(p) \in\left[t_{0}-\delta, t_{0}+\epsilon\right], x(p) \leq x_{0}\right\}\right)} \\
+\gamma\|d u\|_{L_{0}^{2}\left(\left\{p: t(p) \in\left[t_{0}-\delta, t_{0}+\epsilon\right]\right\}\right)}+C^{\prime}\|u\|_{L_{0}^{2}\left(\left\{p: t(p) \in\left[t_{0}-\delta, t_{0}\right]\right\}\right)},
\end{array}
$$

where the norms are relative to the metric $h$.

Proof. We proceed as in the proof of Proposition 2.2, using that the $t$-preimage of the enlargement of the interval by distance $\leq 1$ points is still compact by (PT); we always use $\epsilon<1$ correspondingly. We simply let $\phi=\tilde{\phi} \circ t$, where $\tilde{\phi}$ is the characteristic function of $\left[t_{0}, t_{0}+\epsilon\right]$. Thus $V_{0} \phi$ vanishes near $Y$; at the cost of possibly decreasing $x_{0}$, we may assume that it vanishes in $x<x_{0}$. By (2-3), with $C=\tilde{C}<(n-1) / 2$, if $\psi$ is identically 1 on $\left[0, x_{0} / 4\right)$ and is supported in $\left[0, x_{0} / 2\right)$, then

$$
\|\psi \phi u\|_{L_{0}^{2}(X)} \leq C^{-1}\|\psi V \phi u\|=C^{-1}\|\psi \phi V u\| .
$$

Thus, it remains to give a bound for $\|(1-\psi) u\|_{L_{0}^{2}\left(\left\{p: t(p) \in\left[t_{0}, t_{0}+\epsilon\right]\right)\right\}}$.

Let $\mathscr{Y}$ be the spacelike hypersurface in $X$ given by $t=t_{0}$, with $t_{0} \in I$. Now let $W \in \mathscr{V}_{\mathrm{b}}(X)$ be transversal to $\mathscr{S}$. The standard Poincaré estimate (whose weighted version we prove below in Lemma 2.4) obtained by integrating from $t=t_{0}-\delta$ yields that for $u \in \dot{\mathscr{C}}^{\infty}(X)$ with $\left.u\right|_{t=t_{0}-\delta}=0$,

$$
\|u\|_{L_{0}^{2}\left(\left\{p: t(p) \in\left[t_{0}-\delta, t_{0}+\epsilon\right]\right\}\right)} \leq C^{\prime}(\epsilon+\delta)^{1 / 2}\|W u\|_{L_{0}^{2}\left(\left\{p: t(p) \in\left[t_{0}-\delta, t_{0}+\epsilon\right]\right\}\right)},
$$

with $C^{\prime}(\epsilon+\delta) \rightarrow 0$ as $\epsilon+\delta \rightarrow 0$. Applying this with $u$ supported where $x \in\left(x_{0} / 8, \infty\right)$, we have

$$
\|u\|_{L_{0}^{2}\left(\left\{p: t(p) \in\left[t_{0}-\delta, t_{0}+\epsilon\right]\right\}\right)} \leq C^{\prime \prime}(\epsilon+\delta)^{1 / 2}\|x W u\|_{L_{0}^{2}\left(\left\{p: t(p) \in\left[t_{0}-\delta, t_{0}+\epsilon\right]\right\}\right)},
$$

with $C^{\prime \prime}(\epsilon+\delta) \rightarrow 0$ as $\epsilon+\delta \rightarrow 0$. As we want $0<\delta<\epsilon$, we choose $\epsilon>0$ so that

$$
C^{\prime \prime}(2 \epsilon)^{1 / 2}<\gamma \text {. }
$$


Let $\chi \in \mathscr{C}_{\text {comp }}^{\infty}(\mathbb{R} ;[0,1])$ be identically 1 on $\left[t_{0}, \infty\right)$ and be supported in $\left(t_{0}-\delta, \infty\right)$. Applying (2-6) to $\chi(t) u$, we have

$$
\begin{aligned}
\|u\|_{L_{0}^{2}\left(\left\{p: t(p) \in\left[t_{0}, t_{0}+\epsilon\right]\right\}\right)} \leq C^{\prime \prime}(\epsilon+\delta)^{1 / 2}\|x W u\|_{L_{0}^{2}\left(\left\{p: t(p) \in\left[t_{0}-\delta, t_{0}+\epsilon\right]\right\}\right)} & \\
& +C^{\prime \prime}(\epsilon+\delta)^{1 / 2}\left\|x \chi^{\prime}(t)(W t) u\right\|_{L_{0}^{2}\left(\left\{p: t(p) \in\left[t_{0}-\delta, t_{0}\right]\right\}\right)} .
\end{aligned}
$$

In particular, this can be applied with $u$ replaced by $(1-\psi) u$.

We also need a weighted version of this result. We first recall a Poincaré inequality with weights.

Lemma 2.4. Let $C_{0}>0$. Suppose that $W \in \mathscr{V}_{\mathrm{b}}(X)$ real, $|\operatorname{div} W| \leq C_{0}, \quad 0 \leq \chi \in \mathscr{C}_{\mathrm{comp}}^{\infty}(X)$, and $\chi \leq-\gamma(W \chi)$ for $t \geq t_{0}$, with $0<\gamma<1 /\left(2 C_{0}\right)$. Then there exists $C>0$ such that for $u \in H_{0, \text { loc }}^{1}(X)$ with $t \geq t_{0}$ on $\operatorname{supp} u$,

$$
\int|W \chi||u|^{2} d g \leq C \gamma \int \chi|W u|^{2} d g
$$

Proof. We compute, using $W^{*}=-W-\operatorname{div} W$,

$$
\langle(W \chi) u, u\rangle=\langle[W, \chi] u, u\rangle=\left\langle\chi u, W^{*} u\right\rangle-\langle W u, \chi u\rangle=-\langle\chi u, W u\rangle-\langle W u, \chi u\rangle-\langle\chi u,(\operatorname{div} W) u\rangle
$$

so

$$
\begin{aligned}
\int|W \chi \| u|^{2} d g=-\langle(W \chi) u, u\rangle & \leq 2\left\|\chi^{1 / 2} u\right\|_{L^{2}}\left\|\chi^{1 / 2} W u\right\|_{L^{2}}+C_{0}\left\|\chi^{1 / 2} u\right\|_{L^{2}}^{2} \\
& \leq 2\left(\int \gamma|W \chi \| u|^{2} d g\right)^{1 / 2}\left\|\chi^{1 / 2} W u\right\|_{L^{2}}+C_{0} \int \gamma|W \chi \| u|^{2} d g .
\end{aligned}
$$

Dividing through by $\left(\int|W \chi||u|^{2} d g\right)^{1 / 2}$ and rearranging yields

$$
\left(1-C_{0} \gamma\right)\left(\int|W \chi \| u|^{2} d g\right)^{1 / 2} \leq 2 \gamma^{1 / 2}\left\|\chi^{1 / 2} W u\right\|_{L^{2}} ;
$$

hence the claim follows.

Our Poincaré inequality (which could also be named Hardy, in view of the relationship of (2-1) to the Hardy inequality) is then as follows:

Proposition 2.5. Suppose $V_{0} \in \mathscr{V}(X)$ is real with $\left.V_{0} x\right|_{x=0}=1$ and $V_{0} t \equiv 0$ near $Y$, and let $V \in \mathscr{V}_{\mathrm{b}}(X)$ be given by $V=x V_{0}$. Let I be a compact interval. Let $C<(n-1) / 2$. Then there exist $\epsilon>0, x_{0}>0$, $C^{\prime}>0$ and $\gamma_{0}>0$ such that the following holds.

Suppose $t_{0} \in I$ and $0<\gamma<\gamma_{0}$. Let $\chi_{0} \in \mathscr{C}_{\mathrm{comp}}^{\infty}(\mathbb{R}), \chi=\chi_{0} \circ t$ and $0 \leq \chi_{0} \leq-\gamma \chi_{0}^{\prime}$ on $\left[t_{0}, t_{0}+\epsilon\right]$, with $\chi_{0}$ supported in $\left(-\infty, t_{0}+\epsilon\right]$ and $\delta<\epsilon$. For $u \in H_{0, \mathrm{loc}}^{1}(X)$, one has

$$
\begin{aligned}
\left\|\left|\chi^{\prime}\right|^{1 / 2} u\right\|_{L_{0}^{2}\left(\left\{p: t(p) \in\left[t_{0}, t_{0}+\epsilon\right]\right)\right\} \leq} & C^{-1}\left\|\left|\chi^{\prime}\right|^{1 / 2} V u\right\|_{L_{0}^{2}\left(\left\{p: t(p) \in\left[t_{0}-\delta, t_{0}+\epsilon\right], x(p) \leq x_{0}\right\}\right)} \\
& +C^{\prime} \gamma\left\|\chi^{1 / 2} d u\right\|_{L_{0}^{2}\left(\left\{p: t(p) \in\left[t_{0}-\delta, t_{0}+\epsilon\right]\right\}\right)}+C^{\prime}\|u\|_{L_{0}^{2}\left(\left\{p: t(p) \in\left[t_{0}-\delta, t_{0}\right]\right\}\right)},
\end{aligned}
$$

where the norms are relative to the metric $h$. 
Proof. Let $\mathscr{S}$ be the spacelike hypersurface in $X$ given by $t=t_{0}$, where $t_{0} \in I$. We apply Lemma 2.4 with $W \in \mathscr{V}_{\mathrm{b}}(X)$ transversal to $\mathscr{S}$ as follows.

One has from (2-5) applied with $\phi$ replaced by $\left|\chi^{\prime}\right|^{1 / 2}$ that

$$
\left\|\psi\left|\chi^{\prime}\right|^{1 / 2} u\right\|_{L_{0}^{2}(X)} \leq \tilde{C}^{-1}\left\|\psi\left|\chi^{\prime}\right|^{1 / 2} V u\right\|
$$

We now use Lemma 2.4 with $\chi$ replaced by $\chi \rho^{2}$, with $\rho \equiv 1$ on $\operatorname{supp}(1-\psi)$ and $\rho \in \mathscr{C}_{\text {comp }}^{\infty}\left(X^{\circ}\right)$, to estimate $\left\|(1-\psi)|W \chi|^{1 / 2} u\right\|_{L_{0}^{2}(X)}$. We choose $\rho$ so that in addition $W \rho=0$; this can be done by pulling back a function $\rho_{0}$ from $\mathscr{S}$ under the $W$-flow. We may also assume that $\rho$ is supported where $x \geq x_{0} / 8$ in view of $x \geq x_{0} / 4$ on $\operatorname{supp}(1-\psi)$ (we might need to shorten the time interval we consider, that is, $\epsilon>0$, to accomplish this). Thus, $W\left(\rho^{2} \chi\right)=\rho^{2} W \chi$, and hence

$$
\int \rho^{2}|W \chi \| u|^{2} d g \leq C \gamma \int \rho^{2} \chi|W u|^{2} d g .
$$

Since $x \geq x_{0} / 8$ on supp $\rho$, one can estimate $\int \chi \rho^{2}|W u|^{2} d g$ in terms of $\int \chi|d u|_{H}^{2} d g$ (even though $h$ is a Riemannian 0-metric!), giving the desired result.

\section{Energy estimates}

We recall energy estimates on manifolds without boundary in a form that will be particularly convenient in the next sections. Thus, we work on $X^{\circ}$, equipped with a Lorentz metric $g$ and dual metric $G$; let $\square=\square_{g}$ be the d'Alembertian, so $\sigma_{2}(\square)=G$. We consider a "twisted commutator" with a vector field $V=-\imath Z$, where $Z$ is a real vector field, typically of the form $Z=\chi W$, with $\chi$ a cutoff function. Thus, we compute $\left\langle-\imath\left(V^{*} \square-\square V\right) u, u\right\rangle$ - the point being that the use of $V^{*}$ eliminates zeroth order terms and hence is useful when we work not merely modulo lower order terms.

Note that $-\imath\left(V^{*} \square-\square V\right)$ is a second order, real, self-adjoint operator, so if its principal symbol agrees with that of $d^{*} C d$ for some real self-adjoint bundle endomorphism $C$, then in fact both operators are the same as the difference is zeroth order and vanishes on constants. Correspondingly, there are no zeroth order terms to estimate, which is useful as the latter tend to involve higher derivatives of $\chi$, which in turn tend to be large relative to $d \chi$. The principal symbol in turn is easy to calculate, for the operator is

$$
-\imath\left(V^{*} \square-\square V\right)=-\imath\left(V^{*}-V\right) \square+\imath[\square, V],
$$

whose principal symbol is

$$
-\imath \sigma_{0}\left(V^{*}-V\right) G+H_{G} \sigma_{1}(V)
$$

In fact, it is easy to perform this calculation explicitly in local coordinates $z_{j}$ and dual coordinates $\zeta_{j}$. Let $d g=J|d z|$, so $J=|\operatorname{det} g|^{1 / 2}$. We write the components of the metric tensors as $g_{i j}$ and $G^{i j}$, and $\partial_{j}=\partial_{z_{j}}$ when this does not cause confusion. We also write $Z=\chi W=\sum_{j} Z^{j} \partial_{j}$. In the remainder of this section only, we adopt the standard summation convention. Then

$$
(-\imath Z)^{*}=\imath Z^{*}=-\imath J^{-1} \partial_{j} J Z^{j} \quad \text { and } \quad-\square=J^{-1} \partial_{i} J G^{i j} \partial_{j},
$$


so

$$
\begin{aligned}
-\imath\left(V^{*}-V\right) u & =-\imath\left((-\imath Z)^{*}+\imath Z\right) u=\left(Z^{*}+Z\right) u=\left(-J^{-1} \partial_{j} J Z^{j}+Z^{j} \partial_{j}\right) u \\
& =-J^{-1}\left(\partial_{j} J Z^{j}\right) u=-(\operatorname{div} Z) u, \\
H_{G} & =G^{i j} \zeta_{i} \partial_{z_{j}}+G^{i j} \zeta_{j} \partial_{z_{i}}-\left(\partial_{z_{k}} G^{i j}\right) \zeta_{i} \zeta_{j} \partial_{\zeta_{k}},
\end{aligned}
$$

(the first two terms of $H_{G}$ are the same after summation, but it is convenient to keep them separate); hence

$$
H_{G} \sigma_{1}(V)=G^{i j}\left(\partial_{z_{j}} Z^{k}\right) \zeta_{i} \zeta_{k}+G^{i j}\left(\partial_{z_{i}} Z^{k}\right) \zeta_{j} \zeta_{k}-Z^{k}\left(\partial_{z_{k}} G^{i j}\right) \zeta_{i} \zeta_{j}
$$

Relabeling the indices, we deduce that

$$
-\imath \sigma_{0}\left(V^{*}-V\right) G+H_{G} \sigma_{1}(V)=\left(-J^{-1}\left(\partial_{k} J Z^{k}\right) G^{i j}+G^{i k}\left(\partial_{k} Z^{j}\right)+G^{j k}\left(\partial_{k} Z^{i}\right)-Z^{k} \partial_{k} G^{i j}\right) \zeta_{i} \zeta_{j},
$$

with the first and fourth terms combining into $-J^{-1} \partial_{k}\left(J Z^{k} G^{i j}\right) \zeta_{i} \zeta_{j}$, so

$$
\begin{aligned}
& -\imath\left(V^{*} \square-\square V\right)=d^{*} C d, \quad C_{i j}=g_{i \ell} B_{\ell j} \\
& B_{i j}=-J^{-1} \partial_{k}\left(J Z^{k} G^{i j}\right)+G^{i k}\left(\partial_{k} Z^{j}\right)+G^{j k}\left(\partial_{k} Z^{i}\right),
\end{aligned}
$$

where $C_{i j}$ are the matrix entries of $C$ relative to the basis $\left\{d z_{s}\right\}$ of the fibers of the cotangent bundle.

We now want to expand $B$ using $Z=\chi W$, and separate the terms with $\chi$ derivatives, with the idea being that we choose the derivative of $\chi$ large enough relative to $\chi$ to dominate the other terms. Thus,

$$
\begin{aligned}
B_{i j} & =G^{i k}\left(\partial_{k} Z^{j}\right)+G^{j k}\left(\partial_{k} Z^{i}\right)-J^{-1} \partial_{k}\left(J Z^{k} G^{i j}\right) \\
& =\left(\partial_{k} \chi\right)\left(G^{i k} W^{j}+G^{j k} W^{i}-G^{i j} W^{k}\right)+\chi\left(G^{i k}\left(\partial_{k} Z^{j}\right)+G^{j k}\left(\partial_{k} Z^{i}\right)-J^{-1} \partial_{k}\left(J Z^{k} G^{i j}\right)\right)
\end{aligned}
$$

and multiplying the first term on the right hand side by $\partial_{i} u \overline{\partial_{j} u}$ (and summing over $i, j$ ) gives

$$
\begin{aligned}
E_{W, d \chi}(d u) & =\left(\partial_{k} \chi\right)\left(G^{i k} W^{j}+G^{j k} W^{i}-G^{i j} W^{k}\right) \partial_{i} u \overline{\partial_{j} u} \\
& =(d u, d \chi)_{G} \overline{d u(W)}+d u(W)(d \chi, d u)_{G}-d \chi(W)(d u, d u)_{G},
\end{aligned}
$$

which is twice the sesquilinear stress-energy tensor associated to the wave $u$. This is well known to be positive definite in $d u$, that is, for covectors $\alpha, E_{W, d \chi}(\alpha) \geq 0$ and vanishing if and only if $\alpha=0$, when $W$ and $d \chi$ are both forward timelike for smooth Lorentz metrics, see for example [Taylor 1996, Section 2.7] or [Hörmander 1985, Lemma 24.1.2]. In the present setting, the metric is degenerate at the boundary, but the analogous result still holds, as we show below.

If we replace the wave operator by the Klein-Gordon operator $P=\square+\lambda, \lambda \in \mathbb{C}$, we obtain an additional term

$$
-\imath \lambda\left(V^{*}-V\right)+2 \operatorname{Im} \lambda V=-\imath \operatorname{Re} \lambda\left(V^{*}-V\right)+\operatorname{Im} \lambda\left(V+V^{*}\right)=-\imath \operatorname{Re} \lambda \operatorname{div} V+\operatorname{Im} \lambda\left(V+V^{*}\right)
$$

in $-\imath\left(V^{*} P-P^{*} V\right)$ as compared to (3-1). With $V=-\imath Z, Z=\chi W$, as above, this contributes $-\operatorname{Re} \lambda(W \chi)$ in terms containing derivatives of $\chi$ to $-l\left(V^{*} P-P^{*} V\right)$. In particular, we have

$$
\begin{aligned}
\left\langle-\imath\left(V^{*} P-P^{*} V\right) u, u\right\rangle= & \int E_{W, d \chi}(d u) d g-\operatorname{Re} \lambda\langle(W \chi) u, u\rangle \\
& +\operatorname{Im} \lambda(\langle\chi W u, u\rangle+\langle u, \chi W u\rangle)+\langle\chi R d u, d u\rangle+\left\langle\chi R^{\prime} u, u\right\rangle,
\end{aligned}
$$


where $R \in \mathscr{C}^{\infty}\left(X^{\circ} ; \operatorname{End}\left(T^{*} X^{\circ}\right)\right)$ and $R^{\prime} \in \mathscr{C}^{\infty}\left(X^{\circ}\right)$.

Now suppose that $W$ and $d \chi$ are both timelike (either forward or backward; this merely changes an overall sign). The point of (3-5) is that one controls the left side if one controls $P u$ (in the extreme case, when $P u=0$, it simply vanishes), and one can regard all terms on the right side after $E_{W, d \chi}(d u)$ as terms one can control by a small multiple of the positive definite quantity $\int E_{W, d \chi}(d u) d g$ due to the Poincaré inequality if one arranges that $\chi^{\prime}$ is large relative to $\chi$, and thus one can control $\int E_{W, d \chi}(d u) d g$ in terms of $P u$.

In fact, one does not expect that $d \chi$ will be nondegenerate timelike everywhere: Then one decomposes the energy terms into a region $\Omega_{+}$where one has the desired definiteness, and a region $\Omega_{-}$where this need not hold, and then one can estimate $\int E_{W, d \tilde{\chi}}(d u) d g$ in $\Omega_{+}$in terms of its behavior in $\Omega_{-}$and $P u$. Thus one propagates energy estimates (from $\Omega_{-}$to $\Omega_{+}$), provided one controls $P u$. Of course, if $u$ is supported in $\Omega_{+}$, then one automatically controls $u$ in $\Omega_{-}$, so we are back to the setting that $u$ is controlled by $P u$. This easily gives uniqueness of solutions, and a standard functional analytic argument by duality gives solvability.

It turns out that in the asymptotically AdS case one can proceed similarly, except that the term $\operatorname{Re} \lambda\langle(W \chi) u, u\rangle$ is not negligible any more at $\partial X$, and neither is $\operatorname{Im} \lambda(\langle\chi W u, u\rangle+\langle u, \chi W u\rangle)$. In fact, the $\operatorname{Re} \lambda$ term is the "same size" as the stress energy tensor at $\partial X$; hence the need for an upper bound for it. Meanwhile the $\operatorname{Im} \lambda$ term is even larger; hence the need for the assumption $\operatorname{Im} \lambda=0$ because although $\chi$ is not differentiated (hence in some sense 'small'), $W$ is a vector field that is too large compared to the vector fields the stress energy tensor can estimate at $\partial X$. It is a b-vector field, rather than a 0 -vector field. We explain these concepts now.

\section{Zero-differential operators and b-differential operators}

We start by recalling that $\mathscr{V}_{\mathrm{b}}(X)$ is the Lie algebra of $\mathscr{C}^{\infty}$ vector fields on $X$ tangent to $\partial X$, while $\mathscr{V}_{0}(X)$ is the Lie algebra of $\mathscr{C}^{\infty}$ vector fields vanishing at $\partial X$. Thus, $\mathscr{V}_{0}(X)$ is a Lie subalgebra of $\mathscr{V}_{\mathrm{b}}(X)$. Note also that both $\mathscr{V}_{0}(X)$ and $\mathscr{V}_{\mathrm{b}}(X)$ are $\mathscr{C}^{\infty}(X)$-modules under multiplication from the left, and they act on $x^{k} \mathscr{C}^{\infty}(X)$, in the case of $\mathscr{V}_{0}(X)$ in addition mapping $\mathscr{C}^{\infty}(X)$ into $x \mathscr{C}^{\infty}(X)$. The Lie subalgebra property can be strengthened as follows.

Lemma 4.1. $\mathscr{V}_{0}(X)$ is an ideal in $\mathscr{V}_{\mathrm{b}}(X)$.

Proof. Suppose $V \in \mathscr{V}_{0}(X)$ and $W \in \mathscr{V}_{\mathrm{b}}(X)$. Then, since $V$ vanishes at $\partial X$, there exists $V^{\prime} \in \mathscr{V}(X)$ such that $V=x V^{\prime}$. Thus,

$$
[V, W]=\left[x V^{\prime}, W\right]=[x, W] V^{\prime}+x\left[V^{\prime}, W\right] .
$$

Now, $[x, W]=-W x \in x \mathscr{C}^{\infty}(X)$ since $W$ is tangent to $Y$, and $\left[V^{\prime}, W\right] \in \mathscr{V}(X)$ since $V^{\prime}, W \in \mathscr{V}(X)$; so $[V, W] \in x \mathscr{V}(X)=\mathscr{V}_{0}(X)$.

As usual, $\operatorname{Diff}_{0}(X)$ is the algebra generated by $\mathscr{V}_{0}(X)$, while $\operatorname{Diff}_{\mathrm{b}}(X)$ is the algebra generated by $\mathscr{V}_{\mathrm{b}}(X)$. We combine these in the following definition, originally introduced in [Vasy 2010b] (indeed, even weights $x^{r}$ were allowed there). 
Definition 4.2. Let $\operatorname{Diff}_{0}^{k} \operatorname{Diff}_{\mathrm{b}}^{m}(X)$ be the (complex) vector space of operators on $\dot{\mathscr{C}}^{\infty}(X)$ of the form

$$
\sum P_{j} Q_{j}, \quad P_{j} \in \operatorname{Diff}_{0}^{k}(X), \quad Q_{j} \in \operatorname{Diff}_{\mathrm{b}}^{k}(X),
$$

where the sum is locally finite, and let

$$
\operatorname{Diff}_{0} \operatorname{Diff}_{\mathrm{b}}(X)=\bigcup_{k=0}^{\infty} \bigcup_{m=0}^{\infty} \operatorname{Diff}_{0}^{k} \operatorname{Diff}_{\mathrm{b}}^{m}(X) .
$$

We recall that this space is closed under composition, and that commutators have one lower order in the 0 -sense than products [Vasy 2010b, Lemma 4.5]:

Lemma 4.3. $\operatorname{Diff}_{0} \operatorname{Diff}_{\mathrm{b}}(X)$ is a filtered ring under composition with

$$
A B \in \operatorname{Diff}_{0}^{k+k^{\prime}} \operatorname{Diff}_{\mathrm{b}}^{m+m^{\prime}}(X) \quad \text { if } A \in \operatorname{Diff}_{0}^{k} \operatorname{Diff}_{\mathrm{b}}^{m}(X) \text { and } B \in \operatorname{Diff}_{0}^{k^{\prime}} \operatorname{Diff}_{\mathrm{b}}^{m^{\prime}}(X)
$$

Composition is commutative to leading order in Diff $_{0}$, that is, for $A$ and $B$ as above, with $k+k^{\prime} \geq 1$,

$$
[A, B] \in \operatorname{Diff}_{0}^{k+k^{\prime}-1} \operatorname{Diff}_{\mathrm{b}}^{m+m^{\prime}}(X) .
$$

Here we need an improved property regarding commutators with $\operatorname{Diff}_{\mathrm{b}}(X)$ (which would a priori only gain in the 0 -sense by the preceding lemma). It is this lemma that necessitates the lack of weights on the $\operatorname{Diff}_{\mathrm{b}}(X)$-commutant.

Lemma 4.4. For $A \in \operatorname{Diff}_{\mathrm{b}}^{s}(X)$ and $B \in \operatorname{Diff}_{0}^{k} \operatorname{Diff}_{\mathrm{b}}^{m}(X)$, with $s \geq 1$,

$$
[A, B] \in \operatorname{Diff}_{0}^{k} \operatorname{Diff}_{\mathrm{b}}^{s+m-1}(X) .
$$

Proof. Only the leading terms in terms of Diff $\mathrm{b}_{\mathrm{b}}$ order in both commutants matter for the conclusion, for otherwise the composition result Lemma 4.3 gives the desired conclusion. We again write elements of $\operatorname{Diff}_{0} \operatorname{Diff}_{\mathrm{b}}(X)$ as locally finite sums of products of vector fields and functions, and then, using Lemma 4.3 and expanding the commutators, we are reduced to checking that

(i) $[W, V]=-[V, W] \in \operatorname{Diff}_{0}^{1}(X)$ for $V \in \mathscr{V}_{0}(X)$ and $W \in \mathscr{V}_{\mathrm{b}}(X)$, which follows from Lemma 4.1, and

(ii) $[W, f]=W f \in \mathscr{C}^{\infty}(X)=\operatorname{Diff}_{\mathrm{b}}^{0}(X)$ for $W \in \mathscr{V}_{\mathrm{b}}(X)$ and $f \in \mathscr{C}^{\infty}(X)$.

In both cases thus, the commutator drops b-order by 1 as compared to the product.

Lemma 4.5. For each nonnegative integer $l$ with $l \leq m$,

$$
x^{l} \operatorname{Diff}_{0}^{k} \operatorname{Diff}_{\mathrm{b}}^{m}(X) \subset \operatorname{Diff}_{0}^{k+l} \operatorname{Diff}_{\mathrm{b}}^{m-l}(X) .
$$

Proof. This result is an immediate consequence of $x \mathscr{V}_{\mathrm{b}}(X) \subset x \mathscr{V}(X)=\mathscr{V}_{0}(X)$.

Integer ordered Sobolev spaces, $H_{0, \mathrm{~b}}^{k, m}(X)$ were defined in the introduction. It is immediate from our definitions that for $P \in \operatorname{Diff}_{0}^{r} \operatorname{Diff}_{\mathrm{b}}^{s}(X)$,

$$
P: H_{0, \mathrm{~b}}^{k, m}(X) \rightarrow H_{0, \mathrm{~b}}^{k-r, s-m}(X)
$$


is continuous.

A particular consequence of Lemma 4.4 is that if $V \in \mathscr{V}_{\mathrm{b}}(X), P \in \operatorname{Diff}_{0}^{m}(X)$, then $[P, V] \in \operatorname{Diff}_{0}^{m}(X)$.

We also note that for $Q \in \mathscr{V}_{\mathrm{b}}(X)$, with $Q=-\imath Z$ and $Z$ real, we have $Q^{*}-Q \in \mathscr{C}^{\infty}(X)$, where the adjoint is taken with respect to the $L^{2}=L_{0}^{2}(X)$ inner product. Namely:

Lemma 4.6. Suppose $Q \in \mathscr{V}_{\mathrm{b}}(X)$, with $Q=-\imath Z$ and $Z$ real. Then $Q^{*}-Q \in \mathscr{C}^{\infty}(X)$, and with

$$
Q=a_{0}\left(x D_{x}\right)+\sum a_{j} D_{y_{j}}
$$

we have

$$
Q^{*}-Q=\operatorname{div} Q=J^{-1}\left(D_{x}\left(x a_{0} J\right)+\sum D_{y_{j}}\left(a_{j} J\right)\right)
$$

with the metric density given by $J|d x d y|$, where $J \in x^{-n} \mathscr{C}^{\infty}(X)$.

Proposition 4.7. Suppose $Q \in \mathscr{V}_{\mathrm{b}}(X)$, with $Q=-\imath Z$ and $Z$ real. Then

$$
-\imath\left(Q^{*} \square-\square Q\right)=d^{*} C d
$$

where $C \in \mathscr{C}^{\infty}\left(X ; \operatorname{End}\left({ }^{0} T^{*} X\right)\right)$. In the basis $\left\{d x / x, d y_{1} / x, \ldots, d y_{n-1} / x\right\}$, we have

$$
C_{i j}=\sum_{\ell} g_{i \ell} \sum_{k}\left(-J^{-1} \partial_{k}\left(J a_{k} \hat{G}^{\ell j}\right)+\hat{G}^{\ell k}\left(\partial_{k} a_{j}\right)+\hat{G}^{j k}\left(\partial_{k} a_{\ell}\right)\right) .
$$

Proof. We write

$$
-\imath\left(Q^{*} \square-\square Q\right)=-\imath\left(Q^{*}-Q\right) \square-\imath[Q, \square] \in \operatorname{Diff}_{0}^{2}(X),
$$

and compute the principal symbol, which we check agrees with that of $d^{*} C d$. One way of achieving this is to do the computation over $X^{\circ}$; by continuity if the symbols agree here, they agree on ${ }^{0} T^{*} X$. But over the interior this is the standard computation leading to (3-2); in coordinates $z_{j}$, with dual coordinates $\zeta_{j}$, writing $Z=\sum Z^{j} \partial_{z_{j}}$ and $G=\sum G^{i j} \partial_{z_{i}} \partial_{z_{j}}$, we find both sides have principal symbol

$$
\sum_{i j} B_{i j} \zeta_{i} \zeta_{j}, B_{i j}=\sum_{k}\left(-J^{-1} \partial_{k}\left(J Z^{k} G^{i j}\right)+G^{i k}\left(\partial_{k} Z^{j}\right)+G^{j k}\left(\partial_{k} Z^{i}\right)\right) .
$$

Now both sides of (4-1) are elements of $\operatorname{Diff}_{0}^{2}(X)$, are formally self-adjoint, real, and have the same principal symbol. Thus, their difference is a first order, self-adjoint and real operator; it follows that its principal symbol vanishes, so in fact this difference is zeroth order. Since it annihilates constants (as both sides do), it actually vanishes.

We particularly care about the terms in which the coefficients $a_{j}$ are differentiated, with the idea being that we write $Z=\chi W$, and choose the derivative of $\chi$ large enough relative to $\chi$ to dominate the other terms. Thus, as in (3-4),

$$
B_{i j}=\sum_{k}\left(\partial_{k} \chi\right)\left(G^{i k} W^{j}+G^{j k} W^{i}-G^{i j} W^{k}\right)+\chi \sum_{k}\left(G^{i k}\left(\partial_{k} Z^{j}\right)+G^{j k}\left(\partial_{k} Z^{i}\right)-J^{-1} \partial_{k}\left(J Z^{k} G^{i j}\right)\right)
$$


and multiplying the first term on the right hand side by $\partial_{i} u \overline{\partial_{j} u}$ (and summing over $i, j$ ) gives

$$
\sum_{i, j, k}\left(\partial_{k} \chi\right)\left(G^{i k} W^{j}+G^{j k} W^{i}-G^{i j} W^{k}\right) \partial_{i} u \overline{\partial_{j} u}
$$

which is twice the sesquilinear stress-energy tensor $\frac{1}{2} E_{W, d \chi}(d u)$ associated to the wave $u$. As we mentioned before, this is positive definite when $W$ and $d \chi$ are both forward timelike for smooth Lorentz metrics. In the present setting, the metric is degenerate at the boundary, but the analogous result still holds since

$$
\begin{aligned}
E_{W, d \chi}(d u) & =\sum_{i, j, k}\left(\partial_{k} \chi\right)\left(\hat{G}^{i k} W^{j}+\hat{G}^{j k} W^{i}-\hat{G}^{i j} W^{k}\right)\left(x \partial_{i} u\right) \overline{x \partial_{j} u} \\
& =(x d u, d \chi)_{\hat{G}} \overline{x d u(W)}+x d u(W)(d \chi, x d u)_{\hat{G}}-d \chi(W)(x d u, x d u)_{\hat{G}},
\end{aligned}
$$

so the Lorentzian nondegenerate nature of $\hat{G}$ proves the (uniform) positive definiteness in $x d u$, considered as an element of $T_{q}^{*} X$, and hence in $d u$, regarded as an element of ${ }^{0} T_{q}^{*} X$. Indeed, we recall the quick proof here since we need to improve on this statement to get an optimal result below.

Thus, we wish to show that for $\alpha \in T_{q}^{*} X, W \in T_{q} X, \alpha$ and $W$ forward timelike,

$$
\hat{E}_{W, \alpha}(\beta)=(\beta, \alpha)_{\hat{G}} \overline{\beta(W)}+\beta(W)(\alpha, \beta)_{\hat{G}}-\alpha(W)(\beta, \beta)_{\hat{G}}
$$

is positive definite as a quadratic form in $\beta$. Since replacing $W$ by a positive multiple does not change the positive definiteness, we may assume, as below, that $(W, W)_{\hat{G}}=1$. Then we may choose local coordinates $\left(z_{1}, \ldots, z_{n}\right)$ such that $W=\partial_{z_{n}}$ and $\left.\hat{g}\right|_{q}=d z_{n}^{2}-\left(d z_{1}^{2}+\cdots+d z_{n-1}^{2}\right)$; thus $\left.\hat{G}\right|_{q}=\partial_{z_{n}}^{2}-$ $\left(\partial_{z_{1}}^{2}+\cdots+\partial_{z_{n-1}}^{2}\right)$. Then $\alpha=\sum \alpha_{j} d z_{j}$ being forward timelike means that $\alpha_{n}>0$ and $\alpha_{n}^{2}>\alpha_{1}^{2}+\cdots+\alpha_{n-1}^{2}$. Thus,

$$
\begin{aligned}
\hat{E}_{W, \alpha}(\beta) & =\left(\beta_{n} \alpha_{n}-\sum_{j=1}^{n-1} \beta_{j} \alpha_{j}\right) \bar{\beta}_{n}+\beta_{n}\left(\alpha_{n} \bar{\beta}_{n}-\sum_{j=1}^{n-1} \alpha_{j} \bar{\beta}_{j}\right)-\alpha_{n}\left(\left|\beta_{n}\right|^{2}-\sum_{j=1}^{n-1}\left|\beta_{j}\right|^{2}\right) \\
& =\alpha_{n} \sum_{j=1}^{n}\left|\beta_{j}\right|^{2}-\beta_{n} \sum_{j=1}^{n-1} \alpha_{j} \bar{\beta}_{j}-\sum_{j=1}^{n-1} \beta_{j} \alpha_{j} \bar{\beta}_{n} \\
& \geq \alpha_{n} \sum_{j=1}^{n}\left|\beta_{j}\right|^{2}-2\left|\beta_{n}\right|\left(\sum_{j=1}^{n-1} \alpha_{j}^{2}\right)^{1 / 2}\left(\sum_{j=1}^{n-1}\left|\beta_{j}\right|^{2}\right)^{1 / 2} \\
& \geq \alpha_{n} \sum_{j=1}^{n}\left|\beta_{j}\right|^{2}-2\left|\beta_{n}\right| \alpha_{n}\left(\sum_{j=1}^{n-1}\left|\beta_{j}\right|^{2}\right)^{1 / 2}=\alpha_{n}\left(\left|\beta_{n}\right|-\left(\sum_{j=1}^{n-1}\left|\beta_{j}\right|^{2}\right)^{1 / 2}\right)^{2} \geq 0
\end{aligned}
$$

with the last inequality strict if $\left|\beta_{n}\right| \neq\left(\sum_{j=1}^{n-1}\left|\beta_{j}\right|^{2}\right)^{1 / 2}$, and the preceding one (by the strict forward timelike character of $\alpha$ ) strict if $\beta_{n} \neq 0$ and $\sum_{j=1}^{n-1}\left|\beta_{j}\right|^{2} \neq 0$. It is then immediate that at least one of these inequalities is strict unless $\beta=0$, which is the claimed positive definiteness. 
We claim that we can make a stronger statement if $U \in T_{q} X$ and $\alpha(U)=0$ and $(U, W)_{\hat{g}}=0$ (thus $U$ is necessarily spacelike, that is, $\left.(U, U)_{\hat{g}}<0\right)$ :

$$
\hat{E}_{W, \alpha}(\beta)+c \frac{\alpha(W)}{(U, U)_{\hat{g}}}|\beta(U)|^{2} \quad \text { for } c<1
$$

is positive definite in $\beta$. Indeed, in this case (again assuming $\left.(W, W)_{\hat{g}}=1\right)$ we can choose coordinates as above so that $W=\partial_{z_{n}}$, and so that $U$ is a multiple of $\partial_{z_{1}}$, namely $U=\left(-(U, U)_{\hat{g}}\right)^{1 / 2} \partial_{z_{1}}$, where $\left.\hat{g}\right|_{q}=d z_{n}^{2}-\left(d z_{1}^{2}+\cdots+d z_{n-1}^{2}\right)$. To achieve this, we complete $e_{n}=W$ and $e_{1}=\left(-(U, U)_{\hat{g}}\right)^{-1 / 2} U$ (which are orthogonal by assumption) to a $\hat{g}$ normalized orthogonal basis $\left(e_{1}, e_{2}, \ldots, e_{n}\right)$ of $T_{q} X$, and then choose coordinates so that the coordinate vector fields are given by the $e_{j}$ at $q$. Then $\alpha$ forward timelike means that $\alpha_{n}>0$ and $\alpha_{n}^{2}>\alpha_{1}^{2}+\cdots+\alpha_{n-1}^{2}$, and $\alpha(U)=0$ means that $\alpha_{1}=0$. Thus, with $c<1$,

$$
\begin{aligned}
& \hat{E}_{W, \alpha}(\beta)+c \frac{\alpha(W)}{(U, U)_{\hat{g}}}|\beta(U)|^{2} \\
& \quad=\left(\beta_{n} \alpha_{n}-\sum_{j=2}^{n-1} \beta_{j} \alpha_{j}\right) \bar{\beta}_{n}+\beta_{n}\left(\alpha_{n} \bar{\beta}_{n}-\sum_{j=2}^{n-1} \alpha_{j} \bar{\beta}_{j}\right)-\alpha_{n}\left(\left|\beta_{n}\right|^{2}-\sum_{j=1}^{n-1}\left|\beta_{j}\right|^{2}\right)-c \alpha_{n}\left|\beta_{1}\right|^{2} \\
& \quad \geq(1-c) \alpha_{n}\left|\beta_{1}\right|^{2}+\left(\left(\beta_{n} \alpha_{n}-\sum_{j=2}^{n-1} \beta_{j} \alpha_{j}\right) \bar{\beta}_{n}+\beta_{n}\left(\alpha_{n} \bar{\beta}_{n}-\sum_{j=2}^{n-1} \alpha_{j} \bar{\beta}_{j}\right)-\alpha_{n}\left(\left|\beta_{n}\right|^{2}-\sum_{j=2}^{n-1}\left|\beta_{j}\right|^{2}\right)\right) .
\end{aligned}
$$

On the right hand side the term in the large parentheses is the same kind of expression as in (4-4), with the terms with $j=1$ dropped, and is thus positive definite in $\left(\beta_{2}, \ldots, \beta_{n}\right)$, For $c<1$, the first term is positive definite in $\beta_{1}$, so the left hand side is indeed positive definite as claimed. Rewriting this in terms of $G$ in our setting, we obtain that for $c<1$

$$
E_{W, d \chi}(d u)-c(W \chi)|x U u|^{2}
$$

is positive definite in $d u$, considered an element of ${ }^{0} T_{q}^{*} X$, when $q \in \partial X$, and hence is positive definite sufficiently close to $\partial X$.

We restate the result:

Lemma 4.8. Suppose $q \in \partial X, U, W \in T_{q} X, \alpha \in T_{q}^{*} X, \alpha(U)=0$ and $(U, W)_{\hat{g}}=0$. Then

$$
E_{W, \alpha}(\beta)+c \frac{\alpha(W)}{(U, U)_{\hat{g}}}|\beta(x U)|^{2} \quad \text { for } c<1
$$

is positive definite in $\beta \in{ }^{0} T_{q}^{*} X$.

At this point we modify the choice of our time function $t$ so that we can construct $U$ and $W$ satisfying the requirements of the lemma.

Lemma 4.9. Assume (TF) and (PT). Given $\delta_{0}>0$ and a compact interval $I$, there exists a function $\tau \in \mathscr{C}^{\infty}(X)$ such that $|t-\tau|<\delta_{0}$ for $t \in I, d \tau$ is timelike in the same component of the timelike cone as $d t$, and $\hat{G}(d \tau, d x)=0$ at $x=0$. 
Proof. Let $\chi \in \mathscr{C}_{\text {comp }}^{\infty}([0, \infty))$ be identically 1 near 0 , with $0 \leq \chi \leq 1$ and $\chi^{\prime} \leq 0$, and supported in $[0,1]$. For $\epsilon, \delta>0$ to be specified, let

$$
\tau=t-x \chi\left(x^{\delta} / \epsilon\right) \frac{\hat{G}(d t, d x)}{\hat{G}(d x, d x)} .
$$

Note that $x \leq \epsilon^{1 / \delta}$ on the support of $\chi\left(x^{\delta} / \epsilon\right)$, so if $\epsilon^{1 / \delta}$ is sufficiently small, then $\hat{G}(d x, d x)$ is negative and bounded away from 0 , in view of (PT) and because $\hat{G}(d x, d x)<0$ at $Y$.

At $x=0$,

$$
d \tau=d t-\frac{\hat{G}(d t, d x)}{\hat{G}(d x, d x)} d x,
$$

so $\hat{G}(d \tau, d x)=0$. As already noted, $x \leq \epsilon^{1 / \delta}$ on the support of $\chi\left(x^{\delta} / \epsilon\right)$, so for $t \in I$ with $I$ compact, we have in view of (PT)

$$
|\tau-t| \leq C \epsilon^{1 / \delta}
$$

with $C$ independent of $\epsilon$ and $\delta$. Next,

$$
d \tau=d t-\alpha \gamma d x-\tilde{\alpha} \gamma d x-\beta \mu,
$$

where

$$
\alpha=\chi\left(\frac{x^{\delta}}{\epsilon}\right), \quad \gamma=\frac{\hat{G}(d t, d x)}{\hat{G}(d x, d x)}, \quad \tilde{\alpha}=\delta \frac{x^{\delta}}{\epsilon} \chi^{\prime}\left(\frac{x^{\delta}}{\epsilon}\right), \quad \beta=x \chi\left(\frac{x^{\delta}}{\epsilon}\right), \quad \mu=d\left(\frac{\hat{G}(d t, d x)}{\hat{G}(d x, d x)}\right) .
$$

Now,

$$
\begin{aligned}
\hat{G}(d t-\alpha \gamma d x, d t-\alpha \gamma d x) & =\hat{G}(d t, d t)-2 \alpha \gamma \hat{G}(d t, d x)+\alpha^{2} \gamma^{2} \hat{G}(d x, d x) \\
& =\hat{G}(d t, d t)-\left(2 \alpha-\alpha^{2}\right) \frac{\hat{G}(d t, d x)^{2}}{\hat{G}(d x, d x)},
\end{aligned}
$$

which is $\geq \hat{G}(d t, d t)$ if $2 \alpha-\alpha^{2} \geq 0$, that is, if $\alpha \in[0,2]$. But $0 \leq \alpha \leq 1$, so

$$
\hat{G}(d t-\alpha \gamma d x, d t-\alpha \gamma d x) \geq \hat{G}(d t, d t)>0
$$

that is, $d t-\alpha \gamma d x$ is timelike. Since $d t-\rho \alpha \gamma d x$ is still timelike for $0 \leq \rho \leq 1, d t-\alpha \gamma d x$ is in the same component of timelike covectors as $d t$, that is, it is forward oriented. Next, observe that with $C^{\prime}=\sup s\left|\chi^{\prime}(s)\right|$,

$$
|\tilde{\alpha}| \leq C^{\prime} \delta, \quad \text { and } \quad|\beta| \leq \epsilon^{1 / \delta},
$$

so over compact sets, $\tilde{\alpha} \gamma d x+\beta \mu$ can be made arbitrarily small by first choosing $\delta>0$ sufficiently small and then $\epsilon>0$ sufficiently small. Thus, $\hat{G}(d \tau, d \tau)$ is forward timelike as well. Reducing $\epsilon>0$ further if needed, (4-5) completes the proof.

This lemma can easily be made global.

Lemma 4.10. Assume $(T F)$ and $(P T)$. Given $\delta_{0}>0$ there exists a function $\tau \in \mathscr{C}^{\infty}(X)$ such that $|t-\tau|<\delta_{0}$ for $t \in \mathbb{R}, d \tau$ is timelike in the same component of the timelike cone as $d t$, and $\hat{G}(d \tau, d x)=0$ at $x=0$.

In particular, $\tau$ also satisfies $(T F)$ and $(P T)$. 
Proof. We proceed as above, but let

$$
\tau=t-x \chi\left(\frac{x^{\delta(t)}}{\epsilon(t)}\right) \frac{\hat{G}(d t, d x)}{\hat{G}(d x, d x)} .
$$

We then have two additional terms,

$$
-x^{1-\delta(t)} \delta^{\prime}(t) \log x \frac{x^{\delta(t)}}{\epsilon(t)} \chi^{\prime}\left(\frac{x^{\delta}(t)}{\epsilon(t)}\right) \frac{\hat{G}(d t, d x)}{\hat{G}(d x, d x)} d t \quad \text { and } \quad x \frac{\epsilon^{\prime}(t)}{\epsilon(t)} \frac{x^{\delta(t)}}{\epsilon(t)} \chi^{\prime}\left(\frac{x^{\delta(t)}}{\epsilon(t)}\right) \frac{\hat{G}(d t, d x)}{\hat{G}(d x, d x)} d t
$$

in $d \tau$. Note that $x \leq \epsilon(t)^{1 / \delta(t)}$ on the support of both terms, while $\left(x^{\delta(t)} / \epsilon(t)\right) \chi^{\prime}\left(x^{\delta(t)} / \epsilon(t)\right)$ is uniformly bounded. Thus, if $\delta(t)<1 / 3,\left|\delta^{\prime}(t)\right| \leq 1$, and $\left|\epsilon^{\prime}(t)\right| \leq 1$, the factor in front of $d t$ in both terms is bounded in absolute value by $C \epsilon(t) \hat{G}(d t, d x) / \hat{G}(d x, d x)$. Now for any $k$ there are $\delta_{k}, \epsilon_{k}>0$, which we may assume are in $(0,1 / 3)$ and are decreasing with $k$, such that $\tau$ so defined satisfies on $I=[-k, k]$ all the requirements if $0<\epsilon(t)<\epsilon_{k}, 0<\delta(t)<\delta_{k}$ on $I,\left|\epsilon^{\prime}(t)\right| \leq 1$ and $\left|\delta^{\prime}(t)\right| \leq 1$. But now in view of the bounds on $\epsilon_{k}$ and $\delta_{k}$ it is straightforward to write down $\epsilon(t)$ and $\delta(t)$ with the desired properties, for example, by approximating the piecewise linear function that takes the value $\epsilon_{k}$ at $\pm(k-1)$ for $k \geq 2$, to get $\epsilon(t)$, and similarly with $\delta$, finishing the proof.

From the remainder of this section, we assume that (TF) and (PT) hold. From now on we simply replace $t$ by $\tau$. We let $W=\hat{G}(d t, \cdot)$ and $U_{0}=\hat{G}(d x, \cdot)$. Thus, at $x=0$,

$$
d t\left(U_{0}\right)=\hat{G}(d x, d t)=0 \quad \text { and } \quad\left(U_{0}, W\right)_{\hat{g}}=\hat{G}(d x, d t)=0 .
$$

We extend $\left.U_{0}\right|_{Y}$ to a vector field $U$ such that $U t=0$, that is, $U$ is tangent to the level surfaces of $t$. Then we have on all of $X$,

$$
W(d t)=\hat{G}(d t, d t)>0 \quad \text { and } \quad U(d x)=\hat{G}(d x, d x)<0
$$

on a neighborhood of $Y$, with uniform upper and lower bounds (bounding away from 0 ) for both bounds (4-6) on compact subsets of $X$.

Using Lemma 4.8 and the equations just above, we thus deduce for $\chi=\tilde{\chi} \circ t$ and $c<1$, for $\rho$ in $\mathscr{C}^{\infty}(X)$ identically 1 near $Y$, and supported sufficiently close to $Y$, for $Q=-\imath Z$ and $Z=\chi W$,

$$
\begin{array}{r}
\left\langle-l\left(Q^{*} P-P^{*} Q\right) u, u\right\rangle=\int E_{W, d \chi}(d u) d g-\operatorname{Re} \lambda\langle(W \chi) u, u\rangle \\
+\operatorname{Im} \lambda(\langle\chi W u, u\rangle+\langle u, \chi W u\rangle)+\langle\chi R d u, d u\rangle+\left\langle\chi R^{\prime} u, u\right\rangle \\
=\left\langle\left(\chi^{\prime} A+\chi R\right) d u, d u\right\rangle+\langle c \rho(W \chi) x U u, x U u\rangle-\operatorname{Re} \lambda\langle(W \chi) u, u\rangle \\
+\operatorname{Im} \lambda(\langle\chi W u, u\rangle+\langle u, \chi W u\rangle)+\left\langle\chi R^{\prime} u, u\right\rangle
\end{array}
$$

with $A, R \in \mathscr{C}^{\infty}\left(X ; \operatorname{End}\left({ }^{0} T^{*} X\right)\right), R^{\prime} \in \mathscr{C}^{\infty}(X)$ and $A$ positive definite, all independent of $\chi$. Here $\rho$ is used since $E_{W, d \chi}(d u)-c(W \chi)|x U u|^{2}$ is only positive definite near $Y$.

Fix $t_{0}<t_{0}+\epsilon<t_{1}$. Let $\chi_{0}(s)=e^{-1 / s}$ for $s>0$ and $\chi_{0}(s)=0$ for $s<0$. Let $\chi_{1}$ be in $\mathscr{C}^{\infty}(\mathbb{R})$, be identically 1 on $[1, \infty)$, and vanish on $(-\infty, 0]$. Thus, $s^{2} \chi_{0}^{\prime}(s)=\chi_{0}(s)$ for $s \in \mathbb{R}$. Now consider

$$
\tilde{\chi}(s)=\chi_{0}\left(-\digamma^{-1}\left(s-t_{1}\right)\right) \chi_{1}\left(\left(s-t_{0}\right) / \epsilon\right),
$$


so supp $\tilde{\chi} \subset\left[t_{0}, t_{1}\right]$, and for $s \in\left[t_{0}+\epsilon, t_{1}\right]$ we have

$$
\begin{aligned}
\tilde{\chi}^{\prime} & =-\digamma^{-1} \chi_{0}^{\prime}\left(-\digamma^{-1}\left(s-t_{1}\right)\right), \quad \text { so } \\
\tilde{\chi} & =-\digamma^{-1}\left(s-t_{1}\right)^{2} \tilde{\chi}^{\prime} .
\end{aligned}
$$

For $\digamma>0$ sufficiently large, this is bounded by a small multiple of $\tilde{\chi}^{\prime}$, namely on $\left[t_{0}+\epsilon, t_{1}\right]$

$$
\tilde{\chi}=-\gamma \tilde{\chi}^{\prime} \quad \text { where } \gamma=\left(t_{1}-t_{0}\right)^{2} \digamma^{-1} \text {. }
$$

In particular, for sufficiently large $\digamma$, we have on $\left[t_{0}+\epsilon, t_{1}\right]$

$$
-\left(\chi^{\prime} A+\chi R\right) \geq-\chi^{\prime} A / 2
$$

In addition, by (2-8) and (4-8), for $\operatorname{Re} \lambda<(n-1)^{2} / 4$ and $c^{\prime}>0$ sufficiently close to 1

$$
-\langle\operatorname{Re} \lambda(W \chi) u, u\rangle \leq c^{\prime}\langle\rho(-W \chi) x U u, x U u\rangle+C^{\prime} \digamma^{-1}\left\|\chi^{1 / 2} d u\right\|^{2},
$$

while

$$
\begin{aligned}
\left|\left\langle\chi R^{\prime} u, u\right\rangle\right| & \leq C^{\prime}\left\|\chi^{1 / 2} u\right\|^{2} \text { and } \\
\left\|\chi^{1 / 2} u\right\|^{2} & \leq C^{\prime} \digamma^{-1}\langle(-W \chi) u, u\rangle \leq C^{\prime \prime} \digamma^{-1}\langle(-W \chi) x U u, x U u\rangle+C^{\prime \prime} \digamma^{-2}\left\|\chi^{1 / 2} d u\right\|^{2} .
\end{aligned}
$$

However, $\operatorname{Im} \lambda(\langle\chi W u, u\rangle+\langle u, \chi W u\rangle)$ is too large to be controlled by the stress energy tensor since $W$ is a b-vector field, but not a 0 -vector field. Thus, to control the $\operatorname{Im} \lambda$ term for $t \in\left[t_{0}+\epsilon, t_{1}\right]$, we need to assume that $\operatorname{Im} \lambda=0$. Then, writing $Q u=Q^{*} u+\left(Q-Q^{*}\right) u$ and choosing $\digamma>0$ sufficiently large to absorb the first term on the right hand side of (4-9), we have

$$
\begin{aligned}
\left\langle-\chi^{\prime} A d u, d u\right\rangle / 2 \leq & -\langle-\imath P u, Q u\rangle+\langle\imath P u, Q u\rangle+\gamma\left\langle\left(-\chi^{\prime}\right) d u, d u\right\rangle \\
\leq & 2 C\left\|\chi^{1 / 2} W P u\right\|_{H_{0}^{-1}(X)}\left\|\chi^{1 / 2} u\right\|_{H_{0}^{1}(X)} \\
& \quad+2 C\left\|\left(-\chi^{\prime}\right)^{1 / 2} P u\right\|_{L_{0}^{2}(X)}\left\|\left(-\chi^{\prime}\right)^{1 / 2} u\right\|_{L_{0}^{2}(X)}+C \gamma\left\|\left(-\chi^{\prime}\right)^{1 / 2} d u\right\|^{2} \\
& \leq 2 C \delta^{-1}\left(\|W P u\|_{H_{0}^{-1}(X)}^{2}+\|P u\|_{L_{0}^{2}(X)}^{2}\right) \\
& \quad+2 C \delta\left(\left\|\chi^{1 / 2} u\right\|_{H_{0}^{1}(X)}^{2}+\left\|\left(-\chi^{\prime}\right)^{1 / 2} u\right\|_{L^{2}(X)}^{2}\right)+C \digamma^{-1}\left\|\left(-\chi^{\prime}\right)^{1 / 2} d u\right\|^{2} .
\end{aligned}
$$

For sufficiently small $\delta>0$ and sufficiently large $\digamma>0$ we absorb all but the first parenthesized term on the right hand side into the left hand side by the positive definiteness of $A$ and the Poincaré inequality, Proposition 2.5, to conclude that for $u$ supported in $\left[t_{0}+\epsilon, t_{1}\right]$,

$$
\left\|\left(-\chi^{\prime}\right)^{1 / 2} d u\right\|_{L_{0}^{2}\left(X ;{ }^{*} T^{*} X\right)} \leq C\|P u\|_{H_{0, \mathrm{~b}}^{-1,1}(X)} .
$$

In view of the Poincaré inequality, we have this result:

Lemma 4.11. Suppose $\lambda<(n-1)^{2} / 4, t_{0}<t_{0}+\epsilon<t_{1}$ and $\chi$ is as above. For $u \in \dot{\mathscr{C}}^{\infty}(X)$ supported in $\left[t_{0}+\epsilon, t_{1}\right]$, one has

$$
\left\|\left(-\chi^{\prime}\right)^{1 / 2} u\right\|_{H_{0}^{1}(X)} \leq C\|P u\|_{H_{0, \mathrm{~b}}^{-1,1}(X)}
$$


Remark 4.12. If $I$ is compact then there is $T>0$ such that for $t_{0} \in I$ we can take any $t_{1} \in\left(t_{0}, t_{0}+T\right]$, that is, the time interval over which we can make the estimate is uniform over such compact intervals $I$.

This lemma gives local in time uniqueness immediately; hence iterative application of the lemma, together with Remark 4.12, yields this:

Corollary 4.13. Suppose $\lambda<(n-1)^{2} / 4$. For $f \in H_{0, b, 10 c}^{-1,1}(X)$ supported in $t>t_{0}$, there is at most one $u \in H_{0, \text { loc }}^{1}(X)$ such that $\operatorname{supp} u \subset\left\{p: t(p) \geq t_{0}\right\}$ and $P u=f$.

Estimate (4-11) has another consequence via the standard functional analytic argument.

Lemma 4.14. Suppose $\lambda<(n-1)^{2} / 4$ and $I$ is a compact interval. There is $\sigma>0$ such that for $t_{0} \in I$, and for $f \in H_{0, \text { loc }}^{-1}(X)$ supported in $t>t_{0}$, there exists $u \in H_{0, \mathrm{~b}, \text { loc }}^{1,-1}(X)$, such that

$$
\operatorname{supp} u \subset\left\{p: t(p) \geq t_{0}\right\} \quad \text { and } \quad P u=f \quad \text { in } t<t_{0}+\sigma .
$$

Proof. For any subspace $\mathfrak{X}$ of $\mathscr{C}^{-\infty}(X)$, let $\left.\mathfrak{X}\right|_{\left[\tau_{0}, \tau_{1}\right]}$ consist of elements of $\mathfrak{X}$ restricted to $t \in\left[\tau_{0}, \tau_{1}\right]$, and let $\mathfrak{X}_{\left[\tau_{0}, \tau_{1}\right]}^{\cdot}$ consist of elements of $\mathfrak{X}$ supported in $t \in\left[\tau_{0}, \tau_{1}\right]$. In particular, an element of $\dot{\mathscr{C}}_{\text {comp }}^{\infty}(X)_{\left[\tau_{0}, \tau_{1}\right]}^{\infty}$ vanishes to infinite order at $t=\tau_{0}, \tau_{1}$. Thus, the dot over $\mathscr{C}^{\infty}$ denotes the infinite order vanishing at $\partial X$, while the $\bullet$ denotes the infinite order vanishing at the time boundaries we artificially imposed.

We assume that $f$ is supported in $t>t_{0}+\delta_{0}$. We use Lemma 4.11, with the role of $t_{0}$ and $t_{1}$ reversed (backward in time propagation), and our requirement on $\sigma$ is that it is small enough that the backward version of the lemma is valid with $t_{1}=t_{0}+2 \sigma$. (This can be done uniformly over $I$ by Remark 4.12.) Let $T_{1}=t_{1}-\epsilon$ and $t_{1}$ be such that $t_{0}+\sigma=T_{1}^{\prime}<T_{1}<t_{1}<t_{0}+2 \sigma$. Applying the estimate (4-11), using $P=P^{*}$, with $u$ replaced by $\phi \in \dot{\mathscr{C}}_{\text {comp }}^{\infty}(X)_{\left[t_{0}, T_{1}\right]}^{\cdot}$ with $t_{1}$ in the role of $t_{0}$ there (backward estimate), and with $\tau_{0} \in\left[t_{0}, T_{1}\right)$ in the role of $t_{0}$, we obtain

$$
\left\|\left(\chi^{\prime}\right)^{1 / 2} \phi\right\|_{\left.H_{0}^{1}(X)\right|_{\left[\tau_{0}, T_{1}\right]}} \leq C\left\|P^{*} \phi\right\|_{\left.H_{0, \mathrm{~b}}^{-1,1}(X)\right|_{\left[\tau_{0}, T_{1}\right]}} \text { for } \phi \in \dot{\mathscr{C}}_{\mathrm{comp}}^{\infty}(X)_{\left[\tau_{0}, T_{1}\right]}^{\cdot} .
$$

It is also useful to rephrase this as

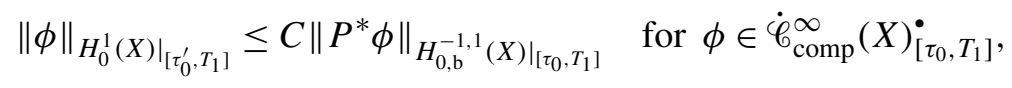

when $\tau_{0}^{\prime}>\tau_{0}$. By (4-13), $P^{*}: \dot{\mathscr{C}}_{\text {comp }}^{\infty}(X)_{\left[t_{0}, T_{1}\right]}^{\bullet} \rightarrow \dot{\mathscr{C}}_{\text {comp }}^{\infty}(X)_{\left[t_{0}, T_{1}\right]}^{\bullet}$ is injective. Define

$$
\left(P^{*}\right)^{-1}: \operatorname{Ran}_{\dot{\mathscr{C}}_{\mathrm{comp}}^{\infty}(X)_{\left[t_{0}, T_{1}\right]}} P^{*} \rightarrow \dot{\mathscr{C}}_{\mathrm{comp}}^{\infty}(X)_{\left[t_{0}, T_{1}\right]}^{\bullet}
$$

by $\left(P^{*}\right)^{-1} \psi$ being the unique $\phi \in \dot{\mathscr{C}}_{\text {comp }}^{\infty}(X)_{\left[t_{0}, T_{1}\right]}^{\bullet}$ such that $P^{*} \phi=\psi$. Now consider the conjugate linear functional on $\operatorname{Ran}_{\dot{\mathscr{C}}_{\text {comp }}^{\infty}(X)_{\left[t_{0}, T_{1}\right]}^{\cdot}}^{\cdot} P^{*}$ given by

$$
\ell: \psi \mapsto\left\langle f,\left(P^{*}\right)^{-1} \psi\right\rangle
$$


In view of (4-13) and the support condition on $f$ (namely, the support is in $t>t_{0}+\delta_{0}$ ) and $\psi$ (the support is in $\left.t \leq T_{1}\right){ }^{2}$

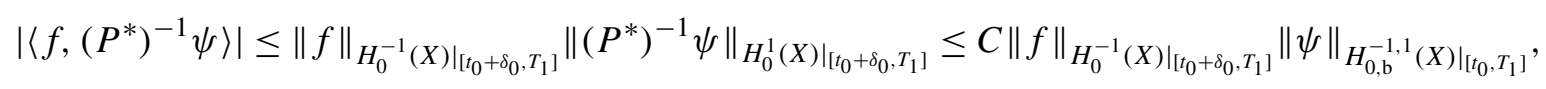

so $\ell$ is a continuous conjugate linear functional if we equip $\operatorname{Ran}_{\dot{\mathscr{C}}_{\text {comp }}^{\infty}(X)_{\left[t_{0}, T_{1}\right]}} P^{*}$ with the $\left.H_{0, \mathrm{~b}}^{-1,1}(X)\right|_{\left[t_{0}, T_{1}\right]}$ norm.

If we did not care about the solution vanishing in $t<t_{0}+\delta_{0}$, we could simply use Hahn-Banach to extend this to a continuous conjugate linear functional $u$ on $H_{0, \mathrm{~b}}^{-1,1}(X)_{\left[t_{0}, T_{1}\right]}^{\cdot}$, which can thus be identified with an element of $\left.H_{0, \mathrm{~b}}^{1,-1}(X)\right|_{\left[t_{0}, T_{1}\right]}$. This would give

$$
P u(\phi)=\langle P u, \phi\rangle=\left\langle u, P^{*} \phi\right\rangle=\ell\left(P^{*} \phi\right)=\left\langle f,\left(P^{*}\right)^{-1} P^{*} \phi\right\rangle=\langle f, \phi\rangle
$$

for $\phi \in \dot{\mathscr{C}}_{\text {comp }}^{\infty}(X)_{\left[t_{0}, T_{1}\right]}^{\bullet}$, so $P u=f$.

We do want the vanishing of $u$ in $\left(t_{0}, t_{0}+\delta_{0}\right)$, that is, when applied to $\phi$ supported in this region. As a first step in this direction, let $\delta_{0}^{\prime} \in\left(0, \delta_{0}\right)$, and note that if

$$
\phi \in \dot{\mathscr{C}}_{\text {comp }}^{\infty}(X)_{\left[t_{0}, t_{0}+\delta_{0}^{\prime}\right)}^{\cdot} \cap \operatorname{Ran}_{\dot{\mathscr{C}}_{\text {comp }}^{\infty}(X)_{\left[t_{0}, T_{1}\right]}^{\cdot}}^{\dot{\theta}^{\prime}} P^{*},
$$

then $\ell(\phi)=0$ directly by (4-15), namely, the right hand side vanishes by the support condition on $f$. Correspondingly, the conjugate linear map $L$ is well defined on the algebraic sum

$$
\dot{\mathscr{C}}_{\mathrm{comp}}^{\infty}(X)_{\left[t_{0}, t_{0}+\delta_{0}^{\prime}\right)}^{\cdot}+\operatorname{Ran}_{\dot{\mathscr{c}}_{\mathrm{comp}}^{\infty}(X)_{\left[t_{0}, T_{1}\right]}^{\cdot}} P^{*}
$$

by

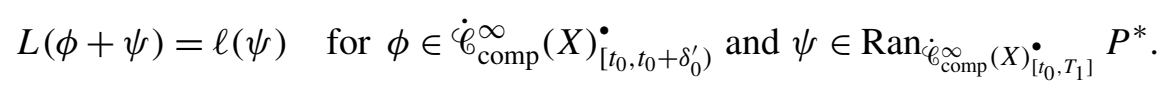

We claim that the functional $L$ is actually continuous when (4-16) is equipped with the $\left.H_{0, \mathrm{~b}}^{-1,1}(X)\right|_{\left[t_{0}, T_{1}\right]}$ norm. This follows from

$$
\left|\left\langle f,\left(P^{*}\right)^{-1} \psi\right\rangle\right| \leq C\|f\|_{\left.H_{0}^{-1}(X)\right|_{\left[t_{0}+\delta_{0}, T_{1}\right]}}\|\psi\|_{\left.H_{0, \mathrm{~b}}^{-1,1}(X)\right|_{\left[t_{0}+\delta_{0}^{\prime}, T_{1}\right]}}
$$

together with

$$
\|\psi\|_{\left.H_{0, \mathrm{~b}}^{-1,1}(X)\right|_{\left[t_{0}+\delta_{0}^{\prime}, T_{1}\right]}} \leq\|\phi+\psi\|_{H_{0, \mathrm{~b}}^{-1,1}(X) \mid\left[t_{0}, T_{1}\right]}
$$

since $\phi$ vanishes on $\left[t_{0}+\delta_{0}^{\prime}, T_{1}\right]$. Correspondingly, by the Hahn-Banach theorem, we can extend $L$ to a continuous conjugate linear map

$$
u: H_{0, \mathrm{~b}}^{-1,1}(X)_{\left[t_{0}, T_{1}\right]}^{:} \rightarrow \mathbb{C},
$$

which can thus by identified with an element of $\left.H_{0, \mathrm{~b}}^{1,-1}(X)\right|_{\left[t_{0}, T_{1}\right]}$. This gives

$$
P u(\phi)=\langle P u, \phi\rangle=\left\langle u, P^{*} \phi\right\rangle=\ell\left(P^{*} \phi\right)=\left\langle f,\left(P^{*}\right)^{-1} P^{*} \phi\right\rangle=\langle f, \phi\rangle
$$

\footnotetext{
${ }^{2}$ We use below that we can regard $f$ as an element of $H_{0}^{-1}(X)_{\left[t_{0}+\delta_{0}, \infty\right)}^{\bullet}$ and $\left(P^{*}\right)^{-1} \psi$ as an element of $H_{0}^{1}(X)_{\left(-\infty, T_{1}\right]}^{\bullet}$, so these can be naturally paired, with the pairing bounded in the appropriate norms. We then write these norms as $\left.H_{0}^{-1}(X)\right|_{\left[t_{0}+\delta_{0}, T_{1}\right]}$ and $\left.H_{0}^{1}(X)\right|_{\left[t_{0}+\delta_{0}, T_{1}\right]}$.
} 
for $\phi \in \dot{\mathscr{C}}_{\text {comp }}^{\infty}(X)_{\left[t_{0}, T_{1}\right]}^{\bullet}$ supported in $\left(t_{0}, T_{1}\right)$, so $P u=f$, and in addition

$$
u(\phi)=0 \quad \text { for } \phi \in \dot{\mathscr{C}}_{\mathrm{comp}}^{\infty}(X)_{\left[t_{0}, t_{0}+\delta_{0}^{\prime}\right]}^{\cdot},
$$

so

$$
t \geq t_{0}+\delta_{0}^{\prime} \quad \text { on } \operatorname{supp} u .
$$

In particular, extending $u$ to vanish on $\left(-\infty, t_{0}+\delta_{0}^{\prime}\right)$, which is compatible with the existing definition in view of (4-17), we have a distribution solving the PDE, defined on $t<T_{1}$, with the desired support condition. In particular, we use a cutoff function $\chi$ that is identically 1 for $t \in\left(-\infty, T_{1}^{\prime}\right]$ and supported on $t \in\left(-\infty, T_{1}\right]$, one has that $\chi u \in H_{0, \mathrm{~b}}^{1,-1}(X)$ and $\chi u$ vanishes for $t<t_{0}+\delta_{0}^{\prime}$ and for $t \geq T_{1}$. Then $P u=f$ on $\left(-\infty, T_{1}^{\prime}\right)$, thus completing the proof.

Proposition 4.15. Suppose $\lambda<(n-1)^{2} / 4$. For $f \in H_{0, \text { loc }}^{-1}(X)$ supported in $t>t_{0}$, there exists $u$ in $H_{0, \mathrm{~b}, \text { loc }}^{1,-1}(X)$ such that $\operatorname{supp} u \subset\left\{p: t(p) \geq t_{0}\right\}$ and $P u=f$.

Proof. We subdivide the timeline into intervals $\left[t_{j}, t_{j+1}\right]$, each of which is sufficiently short so that energy estimates hold even on $\left[t_{j-2}, t_{j+3}\right]$; this can be done in view of the uniform estimates on the length of such intervals over compact subsets. Using a partition of unity, we may assume that $f$ is supported in $\left[t_{k-1}, t_{k+2}\right]$, and we need to construct a global solution of $P u=f$ with $u$ supported in $\left[t_{k-1}, \infty\right)$. First we obtain $u_{k}$ as above solving the PDE on $\left(-\infty, t_{k+2}\right]$ (that is, $P u_{k}-f$ is supported in $\left(t_{k+2}, \infty\right)$ ) and supported in $\left[t_{k-1}, t_{k+3}\right]$. Let $f_{k+1}=P u_{k}-f$; this is thus supported in $\left[t_{k+2}, t_{k+3}\right]$. We next solve $P u_{k+1}=-f_{k+1}$ on $\left(-\infty, t_{k+3}\right]$ with a result supported in $\left[t_{k+1}, t_{k+4}\right]$. Then $P\left(u_{k}+u_{k+1}\right)-f$ is supported in $\left[t_{k+3}, t_{k+4}\right]$, etc. Proceeding inductively and noting that the resulting sum is locally finite, we obtain the solution on all of $X$.

Well-posedness of the solution will follow once we show that for solutions $u \in H_{0, \mathrm{~b}, \mathrm{loc}}^{1, s^{\prime}}(X)$ of $P u=f$, with $f \in H_{0, \mathrm{~b} \text { loc }}^{-1, s}(X)$ supported in $t>t_{0}$, we in fact have $u \in H_{0, \mathrm{~b} \text {,loc }}^{1, s-1}(X)$; indeed, this is a consequence of the propagation of singularities. We state this as a theorem now, recalling the standing assumptions as well:

Theorem 4.16. Assume that $(T F)$ and $(P T)$ hold. Suppose $\lambda<(n-1)^{2} / 4$. For $f \in H_{0, b, l o c}^{-1,1}(X)$ supported in $t>t_{0}$, there exists a unique $u \in H_{0, \text { loc }}^{1}(X)$ such that $\operatorname{supp} u \subset\left\{p: t(p) \geq t_{0}\right\}$ and $P u=f$. Moreover, for $K \subset X$ compact there is $K^{\prime} \subset X$ compact, depending on $K$ and $t_{0}$ only, such that

$$
\left\|\left.u\right|_{K}\right\|_{H_{0}^{1}(X)} \leq\left\|\left.f\right|_{K^{\prime}}\right\|_{H_{0, \mathrm{~b}}^{-1,1}(X)} .
$$

Remark 4.17. While we used $\tau$ of Lemma 4.10 instead of $t$ throughout, the conclusion of this theorem is invariant under this change (since $\delta_{0}>0$ is arbitrary in Lemma 4.10), and thus is actually valid for the original $t$ as well.

Proof. Uniqueness and (4-18) follow from Corollary 4.13 and the estimate (4-12). By Proposition 4.15, this problem has a solution $u \in H_{0, \mathrm{~b}, \text { loc }}^{1,-1}(X)$ with the desired support property. By the propagation of singularities, Theorem 8.8, we know $u \in H_{0, \text { loc }}^{1}(X)$ since $u$ vanishes for $t<t_{0}$. 


\section{Zero-differential operators and b-pseudodifferential operators}

To microlocalize, we need to replace $\operatorname{Diff}_{\mathrm{b}}(X)$ by $\Psi_{\mathrm{b}}(X)$ and $\Psi_{\mathrm{bc}}(X)$. We refer to [Melrose 1993] for a thorough discussion and [Vasy 2008c, Section 2] for a concise introduction to these operator algebras including all the facts that are required here. In particular, the distinction between $\Psi_{\mathrm{b}}(X)$ and $\Psi_{\mathrm{bc}}(X)$ is the same as between $\Psi_{\mathrm{cl}}\left(\mathbb{R}^{n}\right)$ and $\Psi\left(\mathbb{R}^{n}\right)$ of classical, or one step polyhomogeneneous, respectively standard, pseudodifferential operators, that is, elements of the former $\left(\Psi_{\mathrm{b}}(X)\right.$, respectively $\left.\Psi_{\mathrm{cl}}\left(\mathbb{R}^{n}\right)\right)$ are (locally) quantizations of symbols with a full one-step polyhomogeneous asymptotic expansion (also called classical symbols), while those of the latter $\left(\Psi_{\mathrm{bc}}(X)\right.$, respectively $\left.\Psi\left(\mathbb{R}^{n}\right)\right)$ are (locally) quantizations of symbols that merely satisfy symbolic estimates. While the former are convenient since they have homogeneous principal symbols, the latter are more useful when one must use approximations (for example, by smoothing operators), as is often the case below. Before proceeding, we recall that points in the b-cotangent bundle ${ }^{\mathrm{b}} T^{*} X$ of $X$ are of the form

$$
\underline{\xi} \frac{d x}{x}+\sum_{j=1}^{n-1} \underline{\zeta}_{j} d y_{j}
$$

Thus, $(x, y, \underline{\xi}, \underline{\zeta})$ give coordinates on ${ }^{\mathrm{b}} T^{*} X$. If $(x, y, \xi, \zeta)$ are the standard coordinates on $T^{*} X$ induced by local coordinates on $X$, that is, if one-forms are written as $\xi d x+\zeta d y$, then the map $\pi: T^{*} X \rightarrow{ }^{\mathrm{b}} T^{*} X$ is given by $\pi(x, y, \xi, \zeta)=(x, y, x \xi, \zeta)$.

To be a bit more concrete (but again we refer to [Melrose 1993] and [Vasy 2008c, Section 2] for more detail), we can define a large subspace (which in fact is sufficient for our purposes here) of $\Psi_{\mathrm{bc}}^{m}(X)$ and $\Psi_{\mathrm{b}}^{m}(X)$ locally by explicit quantization maps; these can be combined to a global quantization map by a partition of unity as usual. Thus, we have $q=q_{m}: S^{m}\left({ }^{\mathrm{b}} T^{*} X\right) \rightarrow \Psi_{\mathrm{bc}}^{m}(X)$, which restrict to $q: S_{\mathrm{cl}}^{m}\left({ }^{\mathrm{b}} T^{*} X\right) \rightarrow \Psi_{\mathrm{b}}^{m}(X)$, with cl denoting classical symbols. Namely, over a local coordinate chart $U$ with coordinates $(x, y)$, where $y=\left(y_{1}, \ldots, y_{n-1}\right)$, and with $a$ supported in ${ }^{\mathrm{b}} T_{K}^{*} X$ with $K \subset U$ compact, we may take

$$
q(a) u(x, y)=(2 \pi)^{-n} \int e^{l\left(\left(x-x^{\prime}\right) \xi+\left(y-y^{\prime}\right) \cdot \zeta\right)} \phi\left(\frac{x-x^{\prime}}{x}\right) a(x, y, x \xi, \zeta) u\left(x^{\prime}, y^{\prime}\right) d x^{\prime} d y^{\prime} d \xi d \zeta
$$

understood as an oscillatory integral, where $\phi \in \mathscr{C}_{\text {comp }}^{\infty}((-1 / 2,1 / 2))$ is identically 1 near 0 , and the integral in $x^{\prime}$ is over $[0, \infty)$. Note that $\phi$ is irrelevant as far as the behavior of Schwartz kernels near the diagonal is concerned (it is identically 1 there); it simply localizes to a neighborhood of the diagonal. Somewhat inaccurately, one may write $q(a)$ as $a\left(x, y, x D_{x}, D_{y}\right)$, so $a$ is symbolic in b-vector fields; a more accurate way of reflecting this is to change variables, writing $\underline{\xi}=x \underline{\xi}$ and $\underline{\zeta}=\zeta$, so

$$
q(a) u(x, y)=(2 \pi)^{-n} \int e^{l\left(\frac{x-x^{\prime}}{x} \underline{\xi}+\left(y-y^{\prime}\right) \cdot \underline{\zeta}\right)} \phi\left(\frac{x-x^{\prime}}{x}\right) a(x, y, \underline{\xi}, \underline{\zeta}) u\left(x^{\prime}, y^{\prime}\right) \frac{d x^{\prime}}{x} d y^{\prime} d \underline{\xi} d \underline{\zeta} .
$$

With this explicit quantization, the principal symbol $\sigma_{\mathrm{b}, m}(A)$ of $A=q(a)$ is the class $[a]$ of $a$ in $S^{m}\left({ }^{\mathrm{b}} T^{*} X\right) / S^{m-1}\left({ }^{\mathrm{b}} T^{*} X\right)$. If $a$ is classical, this class can be further identified with a homogeneous symbol of degree $m$, that is, an element of $S_{\text {hom }}^{m}\left({ }^{\mathrm{b}} T^{*} X \backslash o\right)$. On the other hand, the operator wave front set $\mathrm{WF}_{\mathrm{b}}^{\prime}(A)$ 
of $A=q(a)$ can be defined by saying that $p \in{ }^{\mathrm{b}} T^{*} X \backslash o$ is not in $\mathrm{WF}_{\mathrm{b}}^{\prime}(A)$ if $p$ has a conic neighborhood $\Gamma$ in ${ }^{\mathrm{b}} T^{*} X \backslash o$ such that $a=a(x, y, \underline{\xi}, \underline{\zeta})$ is rapidly decreasing (that is, is an order $-\infty$ symbol) in $\Gamma$. Thus, $A$ is microlocally order $-\infty$ on the complement of $\mathrm{WF}_{\mathrm{b}}^{\prime}(A)$.

A somewhat better definition of $\Psi_{\mathrm{bc}}(X)$ and $\Psi_{\mathrm{b}}(X)$ is directly in terms of the Schwartz kernels. The Schwartz kernels are well behaved on the b-double space $X_{\mathrm{b}}^{2}=\left[X^{2} ;(\partial X)^{2}\right]$ created by blowing up the corner $(\partial X)^{2}$ in the product space $X^{2}=X \times X$; in particular they are smooth away from the diagonal and vanish to infinite order off the front face. In these terms $\phi$ above localizes to a neighborhood of the diagonal that only intersects the boundary of $X_{\mathrm{b}}^{2}$ in the front face of the blow-up. The equivalence of the two descriptions can be read off directly from (5-1), which shows that the Schwartz kernel is a right b-density valued (this is the factor $\left(d x^{\prime} / x\right) d y^{\prime}$ in (5-1)) distribution conormal to $\left(x-x^{\prime}\right) / x=0$ and $y-y^{\prime}=0$, that is, the lift of the diagonal to $X_{\mathrm{b}}^{2}$.

The space $\Psi_{\mathrm{bc}}(X)$ forms a filtered algebra, so $A B \in \Psi_{\mathrm{bc}}^{m+m^{\prime}}(X)$ for $A \in \Psi_{\mathrm{bc}}^{m}(X)$ and $B \in \Psi_{\mathrm{bc}}^{m^{\prime}}(X)$. In addition, the commutator satisfies $[A, B] \in \Psi_{\mathrm{bc}}^{m+m^{\prime}-1}(X)$, that is, it is one order lower than the product, but there is no gain of decay at $\partial X$. We also recall a crucial lemma from [Vasy 2008c, Section 2]:

Lemma 5.1. For $A \in \Psi_{\mathrm{bc}}^{m}(X)$ and $A \in \Psi_{\mathrm{b}}^{m}(X)$, one has $\left[x D_{x}, A\right] \in x \Psi_{\mathrm{bc}}^{m}(X)$ and $\left[x D_{x}, A\right] \in x \Psi_{\mathrm{b}}^{m}(X)$, respectively.

Proof. The lemma is an immediate consequence of $x D_{x}$ having a commutative normal operator; see [Melrose 1993] for a detailed discussion and [Vasy 2008c, Section 2] for a brief explanation.

For simplicity of notation we state the results from here through Lemma 5.5 for $\Psi_{\mathrm{b}}(X)$; they work equally well if one replaces $\Psi_{\mathrm{b}}(X)$ by $\Psi_{\mathrm{bc}}(X)$ throughout.

Lemma 4.4 still holds with $\operatorname{Diff}_{\mathrm{b}}(X)$ replaced by $\Psi_{\mathrm{b}}(X)$, but without the awkward restriction on positivity of b-orders (which is simply due to the lack of nontrivial negative order differential operators).

Definition 5.2. Let $\operatorname{Diff}_{0}^{k} \Psi_{\mathrm{b}}^{m}(X)$ be the (complex) vector space of operators on $\dot{\mathscr{C}}^{\infty}(X)$ of the form

$$
\sum P_{j} Q_{j}, \quad \text { with } P_{j} \in \operatorname{Diff}_{0}^{k}(X) \text { and } Q_{j} \in \Psi_{\mathrm{b}}^{m}(X),
$$

where the sum is locally finite, and let

$$
\operatorname{Diff}_{0} \Psi_{\mathrm{b}}(X)=\bigcup_{k=0}^{\infty} \bigcup_{m \in \mathbb{R}}^{\infty} \operatorname{Diff}_{0}^{k} \Psi_{\mathrm{b}}^{m}(X) .
$$

We define $\operatorname{Diff}_{0}^{k} \Psi_{\mathrm{bc}}^{m}(X)$ similarly, by replacing $\Psi_{\mathrm{b}}(X)$ by $\Psi_{\mathrm{bc}}(X)$ throughout the definition.

The ring structure (even with a weight $x^{r}$ ) of $\operatorname{Diff}_{0} \Psi_{\mathrm{b}}(X)$ was proved in [Vasy 2010b, Corollary 4.4 and Lemma 4.5], which we recall here. We add to the statements of these results that $\operatorname{Diff}_{0} \Psi_{\mathrm{b}}(X)$ is also closed under adjoints with respect to any weighted nondegenerate b-density, and in particular with respect to a nondegenerate 0-density such as $|d g|$, since both $\operatorname{Diff}_{0}(X)$ and $\Psi_{\mathrm{b}}(X)$ are closed under these adjoints and $(A B)^{*}=B^{*} A^{*}$.

Lemma 5.3. $\operatorname{Diff}_{0} \Psi_{\mathrm{b}}(X)$ is a filtered $*_{\text {-ring }}$ under composition (and adjoints) with

$$
A B \in \operatorname{Diff}_{0}^{k+k^{\prime}} \Psi_{\mathrm{b}}^{m+m^{\prime}}(X) \quad \text { if } A \in \operatorname{Diff}_{0}^{k} \Psi_{\mathrm{b}}^{m}(X) \text { and } B \in \operatorname{Diff}_{0}^{k^{\prime}} \Psi_{\mathrm{b}}^{m^{\prime}}(X)
$$


and

$$
A^{*} \in \operatorname{Diff}_{0}^{k} \Psi_{\mathrm{b}}^{m}(X) \quad \text { if } A \in \operatorname{Diff}_{0}^{k} \Psi_{\mathrm{b}}^{m}(X)
$$

where the adjoint is taken with respect to a (that is, any fixed) nondegenerate 0-density. Moreover, composition is commutative to leading order in Diff $_{0}$, that is, for $A$ and $B$ as above and $k+k^{\prime} \geq 1$,

$$
[A, B] \in \operatorname{Diff}_{0}^{k+k^{\prime}-1} \Psi_{\mathrm{b}}^{m+m^{\prime}}(X) .
$$

Just like for differential operators, we again have a lemma that improves the b-order (rather than merely the 0-order) of the commutator provided one of the commutants is in $\Psi_{\mathrm{b}}(X)$. Again, it is crucial here that there are no weights on $\Psi_{\mathrm{b}}(X)$.

Lemma 5.4. $[A, B] \in \operatorname{Diff}_{0}^{k} \Psi_{\mathrm{b}}^{s+m-1}(X)$ if $A \in \Psi_{\mathrm{b}}^{s}(X)$ and $B \in \operatorname{Diff}_{0}^{k} \Psi_{\mathrm{b}}^{m}(X)$,

Proof. Expanding elements of Diff ${ }_{0}^{k}(X)$ as finite sums of products of vector fields and functions, and using that $\Psi_{\mathrm{b}}(X)$ is commutative to leading order, we need to consider commutators $[f, A]$ for $f \in \mathscr{C}^{\infty}(X)$ and $A \in \Psi_{\mathrm{b}}^{s}(X)$ and show that this is in $\Psi_{\mathrm{b}}^{s-1}(X)$, which is automatic as $\mathscr{C}^{\infty}(X) \subset \Psi_{\mathrm{b}}^{0}(X)$. We also need to consider $[V, A]$ for $V \in \mathscr{V}_{0}(X)$ and $A \in \Psi_{\mathrm{b}}^{s}(X)$ and show that this is in $\operatorname{Diff}_{0}^{1} \Psi_{\mathrm{b}}^{s-1}(X)$, that is,

$$
[V, A]=\sum_{j} W_{j} B_{j}+C_{j} \quad \text { for some } B_{j}, C_{j} \in \Psi_{\mathrm{b}}^{s-1}(X) \text { and } W_{j} \in \mathscr{V}_{0}(X) .
$$

But $V=x V^{\prime}$, where $V^{\prime} \in \mathscr{V}(X)$, and

$$
\left[V^{\prime}, A\right]=\sum_{j} W_{j}^{\prime} B_{j}^{\prime}+C_{j}^{\prime} \quad \text { for some } W_{j}^{\prime} \in \mathscr{V}(X) \text { and } B_{j}^{\prime}, C_{j}^{\prime} \in \Psi_{\mathrm{b}}^{s-1}(X) ;
$$

see [Vasy 2008c, Lemma 2.2]. Meanwhile $B^{\prime \prime}=[x, A] x^{-1} \in \Psi_{\mathrm{b}}^{s-1}(X)$, so

$$
[V, A]=[x, A] V^{\prime}+x\left[V^{\prime}, A\right]=B^{\prime \prime}\left(x V^{\prime}\right)+\sum_{j}\left(x W_{j}^{\prime}\right) B_{j}^{\prime}+x C_{j}^{\prime},
$$

which is of the desired form once the first term is rearranged using Lemma 5.3. That is, explicitly $B^{\prime \prime}\left(x V^{\prime}\right)=\left(x V^{\prime}\right) B^{\prime \prime}+\left[B^{\prime \prime}, x V^{\prime}\right]$, with the last term being an element of $\Psi_{\mathrm{b}}^{s-1}(X)$.

We also have an analogue of Lemma 4.5.

Lemma 5.5. For any integer $l \geq 0$,

$$
x^{l} \operatorname{Diff}_{0}^{k} \Psi_{\mathrm{b}}^{m}(X) \subset \operatorname{Diff}_{0}^{k+l} \Psi_{\mathrm{b}}^{m-l}(X) .
$$

Proof. It suffices to show that $x \Psi_{\mathrm{b}}^{m}(X) \subset \operatorname{Diff}_{0}^{1} \Psi_{\mathrm{b}}^{m-1}(X)$; the rest follows by induction. Also, we may localize and assume that $A$ is supported in a coordinate patch; note that

$$
\Psi_{\mathrm{b}}^{-\infty}(X) \subset \operatorname{Diff}_{0}^{1} \Psi_{\mathrm{b}}^{-\infty}(X)
$$

since $\mathscr{C}^{\infty}(X) \subset \operatorname{Diff}_{0}^{1}(X)$. Thus, let $A \in \Psi_{\mathrm{b}}^{m}(X)$. Then there exist $A_{j} \in \Psi_{\mathrm{b}}^{m-1}(X)$ for $j=0, \ldots, n-1$, and $R \in \Psi_{\mathrm{b}}^{-\infty}(X)$ such that

$$
A=\left(x D_{x}\right) A_{0}+\sum_{j} D_{y_{j}} A_{j}+R
$$


to achieve this, one simply needs to use the ellipticity of $L=\left(x D_{x}\right)^{2}+\sum D_{y_{j}}^{2}$ by constructing a parametrix $G \in \Psi_{\mathrm{b}}^{-2}(X)$ to it, and writing $A=L G A+E A$, with $E \in \Psi_{\mathrm{b}}^{-\infty}(X)$. As $x\left(x D_{x}\right), x D_{y_{j}} \in \mathscr{V}_{0}(X)$, the conclusion follows.

As a consequence of our results thus far, we deduce that $\Psi_{\mathrm{b}}^{0}(X)$ is bounded on $H_{0}^{m}(X)$, as stated already in [Vasy 2010b, Lemma 4.7].

Proposition 5.6. Suppose $m \in \mathbb{Z}$. Any $A \in \Psi_{\mathrm{bc}}^{0}(X)$ with compact support defines a bounded operator on $H_{0}^{m}(X)$, with operator norm bounded by a seminorm of $A$ in $\Psi_{\mathrm{bc}}^{0}(X)$.

Proof. For $m \geq 0$ this is a special case of [Vasy 2010b, Lemma 4.7]. The fact that the operator norm is bounded by a seminorm of $A$ in $\Psi_{\mathrm{bc}}^{0}(X)$ was not explicitly stated there, though follows from the proof. The case $m<0$ follows by duality.

For the convenience of the reader we recall the proof in the case we actually use in this paper, namely $m=1$ (then $m=-1$ follows by duality). Any $A$ as in the statement of the proposition is bounded on $L^{2}(X)$ with the stated properties. Thus, we need to show that if $V \in \mathscr{V}_{0}(X)$, then $V A: H_{0}^{1}(X) \rightarrow L^{2}(X)$. But $V A=A V+[V, A]$ and $[V, A] \in \operatorname{Diff}_{0}^{1} \Psi_{\mathrm{b}}^{-1}(X) \subset \Psi_{\mathrm{b}}^{0}(X)$. Hence $A V: H_{0}^{1}(X) \rightarrow L^{2}(X)$ and $[V, A]: L^{2}(X) \rightarrow L^{2}(X)$, with the claimed norm behavior.

If $q$ is a homogeneous function on ${ }^{\mathrm{b}} T^{*} X \backslash o$, then we again consider the Hamilton vector field $\mathrm{H}_{q}$ associated to it on $T^{*} X^{\circ} \backslash o$. A calculation with change of coordinates shows that in the b-canonical coordinates given above

$$
\mathrm{H}_{q}=\left(\partial_{\underline{\xi}} q\right) x \partial_{x}-\left(x \partial_{x} q\right) \partial_{\underline{\xi}}+(\partial \underline{\zeta} q) \partial_{y}-\left(\partial_{y} q\right) \partial \underline{\zeta},
$$

so $\mathrm{H}_{q}$ extends to a $\mathscr{C}^{\infty}$ vector field on ${ }^{\mathrm{b}} T^{*} X \backslash o$ that is tangent to ${ }^{\mathrm{b}} T_{\partial X}^{*} X$. If $Q \in \Psi_{\mathrm{b}}^{m^{\prime}}(X)$ and $P \in \Psi_{\mathrm{b}}^{m}(X)$, then $[Q, P] \in \Psi_{\mathrm{b}}^{m+m^{\prime}-1}(X)$ has principal symbol

$$
\sigma_{\mathrm{b}, m+m^{\prime}-1}([Q, P])=\frac{1}{l} \mathrm{H}_{q} p .
$$

Using Proposition 5.6 we can define a meaningful $\mathrm{WF}_{\mathrm{b}}$ relative to $H_{0}^{1}(X)$. First we recall the definition of the corresponding global function space from [Vasy 2010b, Section 4]:

For $k \geq 0$ the b-Sobolev spaces relative to $H_{0}^{r}(X)$ are given by ${ }^{3}$

$$
H_{0, \mathrm{~b}, \mathrm{comp}}^{r, k}(X)=\left\{u \in H_{0, \mathrm{comp}}^{r}(X): A u \in H_{0, \text { comp }}^{r}(X) \text { for all } A \in \Psi_{\mathrm{b}}^{k}(X)\right\} .
$$

These can be normed by taking any properly supported elliptic $A \in \Psi_{\mathrm{b}}^{k}(X)$ and letting

$$
\|u\|_{H_{0, b, c o m p}^{r, k}(X)}^{2}=\|u\|_{H_{0}^{r}(X)}^{2}+\|A u\|_{H_{0}^{r}(X)}^{2} .
$$

Although the norm depends on the choice of $A$, for $u$ supported in a fixed compact set, different choices give equivalent norms; see [Vasy 2010b, Section 4] for details in the 0-setting (where supports are not

\footnotetext{
${ }^{3}$ We do not need weighted spaces, unlike in [Vasy 2010b], so we only state the definition in the special case when the weight is identically 1 . On the other hand, we are working on a noncompact space, so we must consider local spaces and spaces of compactly supported functions as in [Vasy 2008c, Section 3]. Note also that we reversed the index convention (which index comes first) relative to [Vasy 2010b], to match the notation for the wave front sets.
} 
an issue), and [Vasy 2008c, Section 3] for an analysis involving supports. We also let $H_{0, \mathrm{~b}, \mathrm{loc}}^{r, k}(X)$ be the subspace of $H_{0, \text { loc }}^{r}(X)$ consisting of $u \in H_{0, \text { loc }}^{r}(X)$ such that $\phi u \in H_{0, \mathrm{~b}, \text { comp }}^{r, k}(X)$ for any $\phi \in \mathscr{C}_{\text {comp }}^{\infty}(X)$.

Here it is also useful to have Sobolev spaces with a negative amount of b-regularity, in a manner completely analogous to [Vasy 2008c, Definition 3.15]:

Definition 5.7. Let $r$ be an integer, $k<0$, and $A \in \Psi_{\mathrm{b}}^{-k}(X)$ be elliptic on ${ }^{\mathrm{b}} S^{*} X$ with proper support. Let $H_{0, \mathrm{~b}, \text { comp }}^{r, k}(X)$ be the space of all $u \in \mathscr{C}^{-\infty}(X)$ of the form $u=u_{1}+A u_{2}$ with $u_{1}, u_{2} \in H_{0, \text { comp }}^{r}(X)$. Let

$$
\|u\|_{H_{0, \mathrm{~b}, \mathrm{comp}}^{r, k}(X)}=\inf \left\{\left\|u_{1}\right\|_{H_{0}^{r}(X)}+\left\|u_{2}\right\|_{H_{0}^{r}(X)}: u=u_{1}+A u_{2}\right\} .
$$

We also let $H_{0, \mathrm{~b}, \text { loc }}^{r, k}(X)$ be the space of all $u \in \mathscr{C}^{-\infty}(X)$ such that $\phi u \in H_{0, \mathrm{~b}, \mathrm{comp}}^{r, k}(X)$ for all $\phi \in \mathscr{C}_{\mathrm{comp}}^{\infty}(X)$.

As discussed for analogous spaces following [Vasy 2008c, Definition 3.15], this definition is independent of the particular $A$ chosen, and different $A$ give equivalent norms for distributions $u$ supported in a fixed compact set $K$. Moreover:

Lemma 5.8. Suppose $r \in \mathbb{Z}$ and $k \in \mathbb{R}$. Any $B \in \Psi_{\mathrm{bc}}^{0}(X)$ with compact support defines a bounded operator on $H_{0, \mathrm{~b}}^{r, k}(X)$, with operator norm bounded by a seminorm of $B$ in $\Psi_{\mathrm{bc}}^{0}(X)$.

Proof. Suppose $k \geq 0$ first. Then for an $A \in \Psi_{\mathrm{b}}^{k}(X)$ as in the definition above,

$$
\|B u\|_{H_{0, \mathrm{~b}, \mathrm{comp}}^{r, k}(X)}^{2}=\|B u\|_{H_{0}^{r}(X)}^{2}+\|A B u\|_{H_{0}^{r}(X)}^{2} .
$$

The first term on the right side is bounded in the desired way due to Proposition 5.6. Letting $G \in \Psi_{\mathrm{b}}^{-k}(X)$ be a properly supported parametrix for $A$ such that $G A=\mathrm{Id}+E$ for $E \in \Psi_{\mathrm{b}}^{-\infty}(X)$, we have $A B u=$ $A B(G A-E) u=(A B G) A u-(A B E) u$, with $A B G \in \Psi_{\mathrm{bc}}^{0}(X)$ and $A B E \in \Psi_{\mathrm{bc}}^{-\infty}(X) \subset \Psi_{\mathrm{bc}}^{0}(X)$. Thus

$$
\|A B u\|_{H_{0}^{r}(X)} \leq C\|A u\|_{H_{0}^{r}(X)}+C\|u\|_{H_{0}^{r}(X)}
$$

by Proposition 5.6, with $C$ bounded by a seminorm of $B$. This completes the proof if $k \geq 0$.

For $k<0$, let $A \in \Psi_{\mathrm{b}}^{-k}(X)$ be as in the definition. If $u=u_{1}+A u_{2}$, and $G \in \Psi_{\mathrm{b}}^{k}(X)$ is a parametrix for $A$ such that $A G=\mathrm{Id}+F$ for $F \in \Psi_{\mathrm{b}}^{-\infty}(X)$, then

$$
B u=B u_{1}+B A u_{2}=B u_{1}+(A G-F) B A u_{2}=B u_{1}+A(G B A) u_{2}-(F B A) u_{2} .
$$

Now, $B, F B A, G B A \in \Psi_{\mathrm{b}}^{0}(X)$ so $B u \in H_{0, \mathrm{~b}, \text { comp }}^{r, k}(X)$. Choosing $u_{1}$ and $u_{2}$ so that

$$
\left\|u_{1}\right\|_{H_{0}^{r}(X)}+\left\|u_{2}\right\|_{H_{0}^{r}(X)} \leq 2\|u\|_{H_{0, \mathrm{~b}, \mathrm{comp}}^{r, k}(X)}
$$

shows the desired continuity, and that the operator norm of $B$ is bounded by a $\Psi_{\mathrm{bc}}^{0}(X)$-seminorm.

Now we define the wave front set relative to $H_{0, \text { loc }}^{r}(X)$. We also allow negative a priori b-regularity relative to this space.

Definition 5.9. Suppose $u \in H_{0, \text { loc }}^{r, k}(X), r \in \mathbb{Z}$ and $k \in \mathbb{R}$. Then $q \in{ }^{\mathrm{b}} T^{*} X \backslash o$ is not in $\mathrm{WF}_{\mathrm{b}}^{r, \infty}(u)$ if there is an $A \in \Psi_{\mathrm{b}}^{0}(X)$ such that $\sigma_{\mathrm{b}, 0}(A)(q)$ is invertible and $Q A u \in H_{0, \text { loc }}^{r}(X)$ for all $Q \in \operatorname{Diff}_{\mathrm{b}}(X)$, that is, if $A u \in H_{0, \mathrm{~b}, \mathrm{loc}}^{r, \infty}(X)$. 
Moreover, $q \in{ }^{\mathrm{b}} T^{*} X \backslash o$ is not in $\mathrm{WF}_{\mathrm{b}}^{r, m}(u)$ if there is an $A \in \Psi_{\mathrm{b}}^{m}(X)$ such that $\sigma_{\mathrm{b}, 0}(A)(q)$ is invertible and $A u \in H_{0, \mathrm{loc}}^{r}(X)$.

Proposition 5.6 implies that $\Psi_{\mathrm{bc}}(X)$ acts microlocally, that is, it preserves $\mathrm{WF}_{\mathrm{b}}$; see [Vasy 2008c, Section 3] for a similar argument. In particular, the proofs for both the qualitative and quantitative version of microlocality go through without any significant changes; one simply replaces the use of [Vasy 2008c, Lemma 3.2] by Proposition 5.6.

Lemma 5.10 (see [Vasy 2008c, Lemma 3.9]). Suppose that $u \in H_{0, \mathrm{~b}, \mathrm{loc}}^{r, k^{\prime}}(X)$ and $B \in \Psi_{\mathrm{bc}}^{k}(X)$. Then $\mathrm{WF}_{\mathrm{b}}^{r, m-k}(B u) \subset \mathrm{WF}_{\mathrm{b}}^{r, m}(u) \cap \mathrm{WF}_{\mathrm{b}}^{\prime}(B)$.

As in [Vasy 2008c, Section 3], the wave front set microlocalizes the "b-singular support relative to $H_{0, \text { loc }}^{r}(X)$ ", meaning this:

Lemma 5.11 (see [Vasy 2008c, Lemma 3.10]). Suppose $u \in H_{0, \mathrm{~b}, \mathrm{loc}}^{r, k}(X), p \in X . I^{\mathrm{b}} S_{p}^{*} X \cap \mathrm{WF}_{\mathrm{b}}^{1, m}(u)=\varnothing$, then in a neighborhood of $p$, $u$ lies in $H_{0, \mathrm{~b}}^{1, m}(X)$, that is, there is $\phi \in \mathscr{C}_{\mathrm{comp}}^{\infty}(X)$ with $\phi \equiv 1$ near $p$ such that $\phi u \in H_{0, \mathrm{~b}}^{1, m}(X)$.

Corollary 5.12 (see [Vasy 2008c, Corollary 3.11]). Suppose $u \in H_{0, \mathrm{~b}, \mathrm{loc}}^{r, k}(X)$ and $\mathrm{WF}_{\mathrm{b}}^{r, m}(u)=\varnothing$. Then $u \in H_{0, \mathrm{~b}, \mathrm{loc}}^{r, m}(X)$.

In particular, if $u \in H_{0, \mathrm{~b}, \mathrm{loc}}^{r, k}(X)$ and $\mathrm{WF}_{\mathrm{b}}^{r, m}(u)=\varnothing$ for all $m$, then $u \in H_{0, \mathrm{~b}, \mathrm{loc}}^{r, \infty}(X)$, that is, $u$ is conormal in that $A u \in H_{0,1 \mathrm{loc}}^{r}(X)$ for all $A \in \operatorname{Diff}_{\mathrm{b}}(X)$ (or indeed $A \in \Psi_{\mathrm{b}}(X)$ ).

Finally, we have the following quantitative bound for which we recall the definition of the wave front set of bounded subsets of $\Psi_{\mathrm{bc}}^{k}(X)$ :

Definition 5.13 (see [Vasy 2008c, Definition 3.12]). Suppose that $\mathscr{B}$ is a bounded subset of $\Psi_{\mathrm{bc}}^{k}(X)$, and $q \in{ }^{\mathrm{b}} S^{*} X$. We say that $q \notin \mathrm{WF}_{\mathrm{b}}^{\prime}(\mathscr{B})$ if there is some $A \in \Psi_{\mathrm{b}}(X)$ that is elliptic at $q$ such that $\{A B: B \in \mathscr{B}\}$ is a bounded subset of $\Psi_{\mathrm{b}}^{-\infty}(X)$.

Lemma 5.14 (see [Vasy 2008c, Lemmas 3.13 and 3.18]). Suppose that $K \subset{ }^{\mathrm{b}} S^{*} X$ is compact and $U$ is a neighborhood of $K$ in ${ }^{\mathrm{b}} S^{*} X$. Let $\tilde{K} \subset X$ compact, and $\tilde{U}$ be a neighborhood of $\tilde{K}$ in $X$ with compact closure. Let $Q \in \Psi_{\mathrm{b}}^{k}(X)$ be elliptic on $K$ with $\mathrm{WF}_{\mathrm{b}}^{\prime}(Q) \subset U$, with Schwartz kernel supported in $\tilde{K} \times \tilde{K}$. Let $\mathscr{B}$ be a bounded subset of $\Psi_{\mathrm{bc}}^{k}(X)$ with $\mathrm{WF}_{\mathrm{b}}^{\prime}(\mathscr{B}) \subset K$ and Schwartz kernel supported in $\tilde{K} \times \tilde{K}$. Then for any $s \leq 0$ there is a constant $C>0$ such that for $B \in \mathscr{B}$ and $u \in H_{0, \mathrm{~b}, \operatorname{loc}}^{r, s}(X)$ with $\mathrm{WF}_{\mathrm{b}}^{r, k}(u) \cap U=\varnothing$, we have

$$
\|B u\|_{H_{0}^{r}(X)} \leq C\left(\|u\|_{H_{0, \mathrm{~b}}^{r, s}(\tilde{U})}+\|Q u\|_{H_{0}^{r}(X)}\right) .
$$

We can use this lemma to obtain uniform bounds for pairings. We call a subset $\mathscr{B}$ of $\operatorname{Diff}_{0}^{m} \Psi_{\mathrm{bc}}^{2 k}(X)$ bounded if its elements are locally finite linear combinations of a fixed, locally finite set of elements of $\operatorname{Diff}_{0}^{m}(X)$ with coefficients that lie in a bounded subset of $\Psi_{\mathrm{bc}}^{2 k}(X)$.

Corollary 5.15. Suppose that $K \subset{ }^{\mathrm{b}} S^{*} X$ is compact and $U$ is a neighborhood of $K$ in ${ }^{\mathrm{b}} S^{*} X$. Let $\tilde{K} \subset X$ be compact, and $\tilde{U}$ be a neighborhood of $\tilde{K}$ in $X$ with compact closure. Let $Q \in \Psi_{\mathrm{b}}^{k}(X)$ be elliptic on $K$ with $\mathrm{WF}_{\mathrm{b}}^{\prime}(Q) \subset U$, with Schwartz kernel supported in $\tilde{K} \times \tilde{K}$. Let $\mathscr{B}$ be a bounded subset of $\operatorname{Diff}_{0}^{2} \Psi_{\mathrm{bc}}^{2 k}(X)$ 
with $\mathrm{WF}_{\mathrm{b}}^{\prime}(\mathscr{B}) \subset K$ and Schwartz kernel supported in $\tilde{K} \times \tilde{K}$. Then there is a constant $C>0$ such that for $B \in \mathscr{B}$ and $u \in H_{0, \mathrm{~b}, \mathrm{loc}}^{1, s}(X)$ with $\mathrm{WF}_{\mathrm{b}}^{1, k}(u) \cap U=\varnothing$, we have

$$
|\langle B u, u\rangle| \leq C\left(\|u\|_{H_{0}^{1}(\tilde{U})}+\|Q u\|_{H_{0}^{1}(X)}\right)^{2} .
$$

Proof. Using Lemma 5.3 we can write $B$ as $\sum B_{i j}^{\prime} P_{i}^{*} R_{j} \Lambda$, where $P_{i}, R_{j} \in \operatorname{Diff}_{0}^{1}(X), \Lambda \in \Psi_{\mathrm{b}}^{k}(X)$ (which we take to be elliptic on $K$, but such that $Q$ is elliptic on $\left.\operatorname{WF}_{\mathrm{b}}^{\prime}(\Lambda)\right), B_{i j}^{\prime}$ lies in a bounded subset $\mathscr{B}^{\prime}$ of $\Psi_{\mathrm{b}}^{k}(X)$ and the sum is finite. Then

$$
\begin{aligned}
|\langle B u, u\rangle| & \leq \sum_{i j}\left|\left\langle R_{j} \Lambda u, P_{i}\left(B_{i j}^{\prime}\right)^{*} u\right\rangle\right| \leq \sum_{i j}\left\|R_{j} \Lambda u\right\|_{L^{2}(X)}\left\|P_{i}\left(B_{i j}^{\prime}\right)^{*} u\right\|_{L^{2}(X)} \\
& \leq \sum_{i j}\|\Lambda u\|_{H_{0}^{1}(X)}\left\|P_{i}\left(B_{i j}^{\prime}\right)^{*} u\right\|_{H_{0}^{1}(X)} \leq \sum C\left(\|u\|_{H_{0, \mathrm{~b}}^{1, s}(\tilde{U})}+\|Q u\|_{H_{0}^{1}(X)}\right)^{2},
\end{aligned}
$$

where in the last step we used Lemma 5.14.

It is useful to note that infinite order b-regularity relative to $L_{0}^{2}(X)$ and $H_{0}^{1}(X)$ are the same.

Lemma 5.16. $\mathrm{WF}_{\mathrm{b}}^{1, \infty}(u)=\mathrm{WF}_{\mathrm{b}}^{0, \infty}(u)$ for $u \in H_{0, \text { loc }}^{1}(X)$.

Proof. The complements of the two sides are the set of points $q \in{ }^{\mathrm{b}} S^{*} X$ for which there exist $A \in \Psi_{\mathrm{b}}^{0}(X)$ (with compactly supported Schwartz kernel, as one may assume) such that $\sigma_{\mathrm{b}, 0}(A)(q)$ is invertible and $L A u \in H_{0}^{1}(X)$, respectively $L A u \in L_{0}^{2}(X)$. Since $H_{0}^{1}(X) \subset L_{0}^{2}(X)$, that $\mathrm{WF}_{\mathrm{b}}^{0, \infty}(u) \subset \mathrm{WF}_{\mathrm{b}}^{1, \infty}(u)$ follows immediately. For the converse, if $L A u \in L_{0}^{2}(X)$ for all $L \in \operatorname{Diff}_{\mathrm{b}}(X)$, then $\operatorname{Diff}_{0}(X) \subset \operatorname{Diff}_{\mathrm{b}}(X)$ shows that $Q L A u \in L_{0}^{2}(X)$ for $Q \in \operatorname{Diff}_{0}^{1}(X)$ and $L \in \operatorname{Diff}_{\mathrm{b}}(X)$, so $L A u \in H_{0}^{1}(X)$, that is, $\mathrm{WF}_{\mathrm{b}}^{1, \infty}(u) \subset \mathrm{WF}_{\mathrm{b}}^{0, \infty}(u)$, completing the proof.

We finally recall that $u \in \mathscr{A}^{k}(X)$, that is, that $u$ is conormal relative to $x^{k} L_{\mathrm{b}}^{2}(X)$, which means that $L u \in x^{k} L_{\mathrm{b}}^{2}(X)$ for all $L \in \operatorname{Diff}_{\mathrm{b}}(X)$, so in particular $u \in x^{k} L_{\mathrm{b}}^{2}(X)$. Thus,

$$
\mathrm{WF}_{\mathrm{b}}^{0, \infty}(u)=\varnothing \quad \text { if and only if } \quad u \in \mathscr{A}^{(n-1) / 2}(X),
$$

in view of $L_{0}^{2}(X)=x^{(n-1) / 2} L_{\mathrm{b}}^{2}(X)$.

\section{Generalized broken bicharacteristics}

We recall the structure of the compressed characteristic set and GBB from [Vasy 2010a, Sections 1 and 2]. In that paper $X$ is a manifold with corners and $k$ is the codimension of the highest codimension corner in the local coordinate chart. Thus, for application to this paper, the reader should take $k=1$ when referring to [Vasy 2010a, Sections 1 and 2]. It is often convenient to work on the cosphere bundle, here ${ }^{\mathrm{b}} S^{*} X$, which is equivalent to working on conic subsets of ${ }^{\mathrm{b}} T^{*} X \backslash o$. In a region where, say,

$$
|\underline{\xi}|<C\left|\underline{\zeta}_{n-1}\right| \text { and }\left|\underline{\zeta}_{j}\right|<C\left|\underline{\zeta}_{n-1}\right| \text { for } j=1, \ldots, n-2 \text {, }
$$

with $C>0$ fixed, we can take

$$
x, y_{1}, \ldots, y_{n-1}, \underline{\hat{\xi}}, \underline{\hat{\zeta}}_{1}, \ldots, \underline{\hat{\zeta}}_{n-2},\left|\underline{\zeta}_{n-1}\right| \quad \text { where } \underline{\hat{\xi}}=\underline{\xi} /\left|\underline{\zeta}_{n-1}\right| \quad \text { and } \quad \underline{\hat{\zeta}}_{j}=\underline{\zeta}_{j} /\left|\underline{\zeta}_{n-1}\right|,
$$


as (projective) local coordinates on ${ }^{\mathrm{b}} T^{*} X \backslash o$, and hence take

$$
x, y_{1}, \ldots, y_{n-1}, \underline{\hat{\xi}}, \underline{\hat{\zeta}}_{1}, \ldots, \underline{\hat{\zeta}}_{n-2}
$$

as local coordinates on the image of this region under the quotient map in ${ }^{\mathrm{b}} S^{*} X$; see [Vasy 2010a, Equation (1.4)].

First, we choose local coordinates more carefully. In arbitrary local coordinates $\left(x, y_{1}, \ldots, y_{n-1}\right)$ on a neighborhood $U_{0}$ of a point on $Y=\partial X$, so that $Y$ is given by $x=0$ inside $x \geq 0$, any symmetric bilinear form on $T^{*} X$ can be written as

$$
\hat{G}(x, y)=A(x, y) \partial_{x} \partial_{x}+\sum_{j} 2 C_{j}(x, y) \partial_{x} \partial_{y_{j}}+\sum_{i, j} B_{i j}(x, y) \partial_{y_{i}} \partial_{y_{j}}
$$

with $A, B, C$ smooth. In view of (1-1), using $x$ given there and coordinates $y_{j}$ on $Y$ pulled by to a collar neighborhood of $Y$ by the product structure, we have in addition

$$
A(0, y)=-1 \quad \text { and } \quad C_{j}(0, y)=0 \text { for all } y,
$$

and $B(0, y)=\left(B_{i j}(0, y)\right)$ is Lorentzian for all $y$. Below we write covectors as

$$
\alpha=\xi d x+\sum_{i=1}^{n-1} \zeta_{i} d y_{i} .
$$

Thus,

$$
\left.\hat{G}\right|_{x=0}=-\partial_{x}^{2}+\sum_{i, j=1}^{n-1} B_{i j}(0, y) \partial_{y_{i}} \partial_{y_{j}},
$$

and hence the metric function, $p(q)=\hat{G}(q, q)$ for $q \in T^{*} X$, is

$$
\left.p\right|_{x=0}=-\xi^{2}+\zeta \cdot B(y) \zeta .
$$

Since $A(0, y)=-1<0$, we see $Y$ is indeed timelike in that the restriction of the dual metric $\hat{G}$ to $N^{*} Y$ is negative definite, for locally the conormal bundle $N^{*} Y$ is given by

$$
\{(x, y, \xi, \zeta): x=0, \zeta=0\} .
$$

We write $h=\zeta \cdot B(y) \zeta$ for the metric function on the boundary. Also, from (6-5),

$$
\mathrm{H}_{p}=-2 \xi \cdot \partial_{x}+\mathrm{H}_{h}+\beta \partial_{\xi}+x V,
$$

where $V$ is a $\mathscr{C}^{\infty}$ vector field in $\mho_{0}=T^{*} U_{0}$ and $\beta$ is a $\mathscr{C}^{\infty}$ function on $U_{0}$.

It is sometimes convenient to improve the form of $B$ near a particular point $p_{0}$, around which the coordinate system is centered. Namely, since $B$ is Lorentzian, we can further arrange it by adjusting the $y_{j}$ coordinates so that

$$
\sum B_{i j}(0,0) \partial_{y_{i}} \partial_{y_{j}}=\partial_{y_{n-1}}^{2}-\sum_{i<n-1} \partial_{y_{i}}^{2} .
$$


We now recall from the introduction that $\pi: T^{*} X \rightarrow{ }^{\mathrm{b}} T^{*} X$ is the natural map corresponding to the identification of a section of $T^{*} X$ as a section of ${ }^{\mathrm{b}} T^{*} X$, and in local coordinates $\pi$ is given by

$$
\pi(x, y, \xi, \zeta)=(x, y, x \xi, \zeta)
$$

Moreover, the image under $\pi$ of the characteristic set $\Sigma \subset T^{*} X \backslash o$, given by

$$
\Sigma=\left\{q \in T^{*} X: p(q)=0\right\},
$$

is the compressed characteristic set $\dot{\Sigma}=\pi(\Sigma)$. Note that (6-5) gives that

$$
\dot{\Sigma} \cap U_{0} \cap{ }^{\mathrm{b}} T_{Y}^{*} X=\{(0, y, 0, \underline{\zeta}): 0 \leq \underline{\zeta} \cdot B(y) \underline{\zeta}, \underline{\zeta} \neq 0\} .
$$

In particular, in view of (6-7), $\dot{\Sigma} \cap u_{0}$ lies in the region (6-1), at least after we possibly shrink $U_{0}$ (recall that $\left.U_{0}=T^{*} U_{0}\right)$, as we assume from now. We also remark that, using (6-6),

$$
\left.\pi_{*}\right|_{(x, y, \xi, \zeta)} \mathrm{H}_{p}=-2 \xi \cdot\left(\partial_{x}+\xi \partial_{\underline{\xi}}\right)+\mathrm{H}_{h}+x \beta \partial_{\underline{\xi}}+x \pi_{*} V,
$$

and correspondingly

$$
\left.\mathrm{H}_{p} \pi^{*} \underline{\xi}\right|_{x=0}=-2 \xi^{2}=2(p-\zeta \cdot B(y) \zeta)=-2 \zeta \cdot B(y) \zeta, \quad \text { where }(0, y, \xi, \zeta) \in \Sigma .
$$

As we already noted, $\underline{\zeta}_{n-1}$ cannot vanish on $\dot{\Sigma} \cap U_{0}$, so

$$
\begin{aligned}
\left.\mathrm{H}_{p} \pi^{*}\left(\underline{\xi} /\left|\underline{\zeta}_{n-1}\right|\right)\right|_{x=0} & =-2\left|\zeta_{n-1}\right|^{-1} \xi^{2}-\left.x \xi\left|\zeta_{n-1}\right|^{-2}\left(\mathrm{H}_{h}\left|\zeta_{n-1}\right|\right)\right|_{x=0} \\
& =-2\left|\zeta_{n-1}\right|^{-1} \zeta \cdot B(y) \zeta,(0, y, \xi, \zeta) \in \Sigma .
\end{aligned}
$$

To better understand the generalized broken bicharacteristics for $\square$, we divide $\dot{\Sigma}$ into two subsets. We thus define the glancing set $\varphi$ as the set of points in $\dot{\Sigma}$ whose preimage under $\hat{\pi}=\left.\pi\right|_{\Sigma}$ consists of a single point, and define the hyperbolic set $\mathscr{H}$ as its complement in $\dot{\Sigma}$. Thus, ${ }^{\mathrm{b}} T_{X^{\circ}}^{*} X \cap \dot{\Sigma} \subset \mathscr{G}$ since $\pi$ is a diffeomorphism on $T_{X^{\circ}}^{*} X$, while $q \in \dot{\Sigma} \cap{ }^{\mathrm{b}} T_{Y}^{*} X$ lies in $\mathscr{G}$ if and only if on $\hat{\pi}^{-1}(\{q\}), \xi=0$. More explicitly, with the notation of (6-8),

$$
\begin{aligned}
& \mathscr{G} \cap \mathcal{U}_{0} \cap{ }^{\mathrm{b}} T_{Y}^{*} X=\{(0, y, 0, \underline{\zeta}): \underline{\zeta} \cdot B(y) \underline{\zeta}=0, \underline{\zeta} \neq 0\}, \\
& \mathscr{H} \cap U_{0} \cap{ }^{\mathrm{b}} T_{Y}^{*} X=\{(0, y, 0, \underline{\zeta}): \underline{\zeta} \cdot B(y) \underline{\zeta}>0, \underline{\zeta} \neq 0\} .
\end{aligned}
$$

Thus, $\mathscr{G}$ corresponds to generalized broken bicharacteristics that are tangent to $Y$ in view of the vanishing of $\xi$ at $\hat{\pi}^{-1}(\mathscr{G})$ (recall that the $\partial_{x}$ component of $\mathrm{H}_{p}$ is $-2 \xi$ ), while $\mathscr{H}$ corresponds to generalized broken bicharacteristics that are normal to $Y$. Note that if $Y$ is one-dimensional (hence $X$ is 2-dimensional), then $\underline{\zeta} \cdot B(y) \underline{\zeta}=0$ necessarily implies $\underline{\zeta}=0$, so in fact $\varphi \cap^{\mathrm{b}} T_{Y}^{*} X=\varnothing$, and hence there are no glancing rays.

We next make the role of $\mathscr{G}$ and $\mathscr{H}$ more explicit, which explains the relevant phenomena better. A characterization of GBB, which is equivalent to Definition 1.1, is this:

Lemma 6.1 (see the discussion in [Vasy 2005, Section 1] after the statement of Definition 1.1). A continuous map $\gamma: I \rightarrow \dot{\Sigma}$, where $I \subset \mathbb{R}$ is an interval, is a $G B B$ (in the analytic sense that we use here) if and only if it satisfies the following requirements: 
(i) If $q_{0}=\gamma\left(s_{0}\right) \in \mathscr{G}$, then for all $f \in \mathscr{C}^{\infty}\left({ }^{\mathrm{b}} T^{*} X\right)$,

$$
\frac{d}{d s}(f \circ \gamma)\left(s_{0}\right)=\mathrm{H}_{p}\left(\pi^{*} f\right)\left(\tilde{q}_{0}\right) \text { where } \tilde{q}_{0}=\hat{\pi}^{-1}\left(q_{0}\right) .
$$

(ii) If $q_{0}=\gamma\left(s_{0}\right) \in \mathscr{H}$, then there exists $\epsilon>0$ such that

$$
\gamma(t) \notin^{\mathrm{b}} T_{Y}^{*} X \quad \text { if } \quad 0<\left|s-s_{0}\right|<\epsilon \text { for } s \in I .
$$

The idea of the proof of this lemma is that at $\mathscr{G}$, the requirement in (i) is equivalent to Definition 1.1 since $\hat{\pi}^{-1}\left(q_{0}\right)$ contains a single point. On the other hand, at $\mathscr{H}$, the requirement in (ii) follows from Definition 1.1 applied to the functions $f= \pm \xi$, using (6-10), to conclude that $\xi$ is strictly decreasing at $\mathscr{H}$ along GBB. Since one has $\underline{\xi}=0$ on $\dot{\Sigma} \cap\{x=0\}$, we have for a GBB $\gamma$ through $\gamma\left(s_{0}\right)=q_{0} \in \mathscr{H}$, on a punctured neighborhood of $s_{0}$, that $\underline{\xi}(\gamma(s)) \neq 0$, so $\gamma(s) \notin^{\mathrm{b}} T_{Y}^{*} X$ (since $\gamma(s) \in \dot{\Sigma}$ ). For the converse direction at $\mathscr{H}$ we refer to [Lebeau 1997]; see [Vasy 2005, Section 1] for details.

\section{Microlocal elliptic regularity}

We first note the form of $\square$ with commutator calculations in mind. Rather than thinking of the tangential terms $x D_{y}$ as "too degenerate", we think of $x D_{x}$ as "too singular" in that it causes the failure of $\square$ to lie in $x^{2} \operatorname{Diff}_{\mathrm{b}}^{2}(X)$. This makes the calculations rather analogous to the conformal case, and also it facilitates the use of the symbolic machinery for b-pseudodifferential operators (b-PsDOs).

Proposition 7.1. On a collar neighborhood of $Y$, the form of $\square$ is

$$
-\left(x D_{x}\right)^{*} \alpha\left(x D_{x}\right)+\left(x D_{x}\right)^{*} M^{\prime}+M^{\prime \prime}\left(x D_{x}\right)+\tilde{P},
$$

with

$$
\begin{array}{rr}
\alpha-1 & \in x \mathscr{C}^{\infty}(X), \quad M^{\prime}, M^{\prime \prime} \in x^{2} \operatorname{Diff}_{\mathrm{b}}^{1}(X) \subset x \operatorname{Diff}_{0}^{1}(X), \\
\tilde{P} & \in x^{2} \operatorname{Diff}_{\mathrm{b}}^{2}(X), \quad \tilde{P}-x^{2} \square_{h} \in x^{3} \operatorname{Diff}_{\mathrm{b}}^{2}(X) \subset x \operatorname{Diff}_{0}^{2}(X),
\end{array}
$$

where $\square_{h}$ is the d'Alembertian of the conformal metric on the boundary (extended to a neighborhood of $Y$ using the collar structure).

Proof. Writing the coordinates as $\left(z_{1}, \ldots, z_{n}\right)$, the operator $\square_{g}$ is given by

$$
\square_{g}=\sum_{i j} D_{z_{i}}^{*} G_{i j} D_{z_{j}}
$$

with adjoints taken with respect to $d g=|\operatorname{det} g|^{1 / 2}\left|d z_{1} \cdots d z_{n}\right|$. With $z_{j}=y_{j}$ for $j=1, \ldots, n-1$ and $z_{n}=x$, this can be rewritten as

$$
\begin{aligned}
\square_{g} & =\sum_{i j}\left(x D_{z_{i}}\right)^{*} \hat{G}_{i j}\left(x D_{z_{j}}\right) \\
& =\left(x D_{x}\right)^{*} \hat{G}_{n n}\left(x D_{x}\right)+\sum_{j=1}^{n-1}\left(x D_{x}\right)^{*} \hat{G}_{n j}\left(x D_{y_{j}}\right)+\sum_{j=1}^{n-1}\left(x D_{y_{j}}\right)^{*} \hat{G}_{j n}\left(x D_{y_{j}}\right)+\sum_{i, j=1}^{n-1}\left(x D_{y_{i}}\right)^{*} \hat{G}_{i j}\left(x D_{y_{j}}\right) .
\end{aligned}
$$


Since $\hat{G}_{n n}+1 \in x \mathscr{C}^{\infty}(X)$, we find that $\alpha-1 \in x \mathscr{C}^{\infty}(X)$ by taking $\alpha=-\hat{G}_{n n}$. Since $\hat{G}_{j n}, \hat{G}_{n j} \in x \mathscr{C}^{\infty}(X)$, we find $M^{\prime}, M^{\prime \prime} \in x^{2} \operatorname{Diff}_{\mathrm{b}}^{1}(X)$ by taking $M^{\prime}=\sum_{j=1}^{n-1} \hat{G}_{n j}\left(x D_{y_{j}}\right)$ and $M^{\prime \prime}=\sum_{j=1}^{n-1}\left(x D_{y_{j}}\right)^{*} \hat{G}_{j n}$. Finally,

$$
\tilde{P}=\sum_{i j=1}^{n-1}\left(x D_{y_{i}}\right)^{*} \hat{G}_{i j}\left(x D_{y_{j}}\right) \in x^{2} \operatorname{Diff}_{\mathrm{b}}^{2}(X) .
$$

Modulo $x^{3} \operatorname{Diff}_{\mathrm{b}}^{2}(X)$, we can pull out the factors of $x$ and restrict $\hat{G}_{i j}$ to $Y$. Therefore $\tilde{P}$ differs from $x^{2} \square_{h}=x^{2} \sum D_{y_{i}}^{*} h_{i j} D_{y_{j}}$ by an element of $x^{3} \operatorname{Diff}_{\mathrm{b}}^{2}(X)$, completing the proof.

We next state the lemma regarding Dirichlet form that is of fundamental use in both the elliptic and hyperbolic/glancing estimates. Below the main assumption is that $P=\square_{g}+\lambda$, with $\square_{g}$ as in (7-1). We first recall the notation for local norms:

Remark 7.2. Since $X$ is noncompact and our results are microlocal, we may always fix a compact set $\tilde{K} \subset X$ and assume that all PsDOs have Schwartz kernel supported in $\tilde{K} \times \tilde{K}$. We also let $\tilde{U}$ be a neighborhood of $\tilde{K}$ in $X$ such that $\tilde{U}$ has compact closure, and use the $H_{0}^{1}(\tilde{U})$ norm in place of the $H_{0}^{1}(X)$ norm to accommodate $u \in H_{0, \text { loc }}^{1}(X)$. (We may instead take $\phi \in \mathscr{C}_{\text {comp }}^{\infty}(\tilde{U})$ identically 1 in a neighborhood of $\tilde{K}$, and use $\|\phi u\|_{H_{0}^{1}(X)}$.) Below we use the notation $\|\cdot\|_{H_{0, \text { loc }}^{1}(X)}$ for $\|\cdot\|_{H_{0}^{1}(\tilde{U})}$ to avoid having to specify $\tilde{U}$. We also use $\|v\|_{H_{0, \mathrm{loc}}^{-1}(X)}$ for $\|\phi v\|_{H_{0}^{-1}(X)}$.

Lemma 7.3 (see [Vasy 2008c, Lemma 4.2]). Suppose that $K \subset{ }^{\mathrm{b}} S^{*} X$ is compact, $U \subset{ }^{\mathrm{b}} S^{*} X$ is open, and $K \subset U$. Suppose that $\mathscr{A}=\left\{A_{r}: r \in(0,1]\right\}$ is a bounded family of PsDOs in $\Psi_{\mathrm{bc}}^{s}(X)$ with $\mathrm{WF}_{\mathrm{b}}^{\prime}(\mathscr{A}) \subset K$, and with $A_{r} \in \Psi_{\mathrm{b}}^{s-1}(X)$ for $r \in(0,1]$. Then there are $G \in \Psi_{\mathrm{b}}^{s-1 / 2}(X)$ and $\tilde{G} \in \Psi_{\mathrm{b}}^{s+1 / 2}(X)$ with $\mathrm{WF}_{\mathrm{b}}^{\prime}(G), \mathrm{WF}_{\mathrm{b}}^{\prime}(\tilde{G}) \subset U$ and $C_{0}>0$ such that for $r \in(0,1]$ and $u \in H_{0, \mathrm{~b}, \mathrm{loc}}^{1, k}(X)$ (here $\left.k \leq 0\right)$ with neither $\mathrm{WF}_{\mathrm{b}}^{1, s-1 / 2}(u)$ nor $\mathrm{WF}_{\mathrm{b}}^{-1, s+1 / 2}(P u)$ intersecting $U$, we have

$$
\left|\left\langle d A_{r} u, d A_{r} u\right\rangle_{G}+\lambda\left\|A_{r} u\right\|^{2}\right| \leq C_{0}\left(\|u\|_{H_{0, b, \mathrm{loc}}^{1, k}(X)}^{2}+\|G u\|_{H_{0}^{1}(X)}^{2}+\|P u\|_{H_{0, b, \mathrm{loc}}^{-1, k}(X)}^{2}+\|\tilde{G} P u\|_{H_{0}^{-1}(X)}^{2}\right) .
$$

Remark 7.4. The point of this lemma is $G$ is $1 / 2$ order lower $(s-1 / 2$ versus $s)$ than the family $A$. We will later take the limit $r \rightarrow 0$ to gain control of the Dirichlet form evaluated on $A_{0} u$, where $A_{0} \in \Psi_{\mathrm{bc}}^{s}(X)$, in terms of lower order information.

The role of $A_{r}$ for $r>0$ is to regularize such an argument, that is, to make sure various terms in a formal computation, in which one uses $A_{0}$ directly, actually make sense.

The main difference with [Vasy 2008c, Lemma 4.2] is that $\lambda$ is not negligible.

Proof. We have $A_{r} u \in H_{0}^{1}(X)$ for $r \in(0,1]$, so

$$
\left\langle d A_{r} u, d A_{r} u\right\rangle+\lambda\left\|A_{r} u\right\|^{2}=\left\langle P A_{r} u, A_{r} u\right\rangle .
$$

Here the right side is the pairing of $H_{0}^{-1}(X)$ with $H_{0}^{1}(X)$, so by writing $P A_{r}=A_{r} P+\left[P, A_{r}\right]$, it can be estimated by

$$
\left|\left\langle A_{r} P u, A_{r} u\right\rangle\right|+\left|\left\langle\left[P, A_{r}\right] u, A_{r} u\right\rangle\right| .
$$


The lemma is thus proved if we show that the first term of (7-2) is bounded by

$$
C_{0}^{\prime}\left(\|u\|_{H_{0, \mathrm{~b}, \mathrm{loc}}^{1, k}(X)}^{2}+\|G u\|_{H_{0}^{1}(X)}^{2}+\|P u\|_{H_{0, \mathrm{~b}, \mathrm{loc}}^{-1, k}(X)}^{2}+\|\tilde{G} P u\|_{H_{0}^{-1}(X)}^{2}\right),
$$

the second term is bounded by $C_{0}^{\prime \prime}\left(\|u\|_{H_{0, b, \text { loc }}^{1, k}(X)}^{2}+\|G u\|_{H_{0}^{1}(X)}^{2}\right)$. (Recall that the "local" norms were defined in Remark 7.2.)

The first term is straightforward to estimate. Let $\Lambda \in \Psi_{\mathrm{b}}^{-1 / 2}(X)$ be elliptic with $\Lambda^{-} \in \Psi_{\mathrm{b}}^{1 / 2}(X)$ a parametrix, so that

$$
E=\Lambda \Lambda^{-}-\mathrm{Id} \quad \text { and } \quad E^{\prime}=\Lambda^{-} \Lambda-\mathrm{Id} \in \Psi_{\mathrm{b}}^{-\infty}(X)
$$

Then

$$
\left\langle A_{r} P u, A_{r} u\right\rangle=\left\langle\left(\Lambda \Lambda^{-}-E\right) A_{r} P u, A_{r} u\right\rangle=\left\langle\Lambda^{-} A_{r} P u, \Lambda^{*} A_{r} u\right\rangle-\left\langle A_{r} P u, E^{*} A_{r} u\right\rangle .
$$

Since $\Lambda^{-} A_{r}$ is uniformly bounded in $\Psi_{\mathrm{bc}}^{s+1 / 2}(X)$ and $\Lambda^{*} A_{r}$ is uniformly bounded in $\Psi_{\mathrm{bc}}^{s-1 / 2}(X)$, we have $\left\langle\Lambda^{-} A_{r} P u, \Lambda^{*} A_{r}\right\rangle$ is uniformly bounded, with a bound like (7-3) using Cauchy-Schwartz and Lemma 5.14. Indeed, by Lemma 5.14, if we choose any $G \in \Psi_{\mathrm{b}}^{s-1 / 2}(X)$ that is elliptic on $K$, there is a constant $C_{1}>0$ such that

$$
\left\|\Lambda^{*} A_{r} u\right\|_{H_{0}^{1}(X)}^{2} \leq C_{1}\left(\|u\|_{H_{0, b, \mathrm{loc}}^{1, k}(X)}^{2}+\|G u\|_{H_{0}^{1}(X)}^{2}\right) .
$$

Similarly, by Lemma 5.14 and its analogue for $\mathrm{WF}_{\mathrm{b}}^{-1, s}$, if we choose any $\tilde{G} \in \Psi_{\mathrm{b}}^{s+1 / 2}(X)$ that is elliptic on $K$, there is a constant $C_{1}^{\prime}>0$ such that

$$
\left\|\Lambda^{-} A_{r} P u\right\|_{H_{0}^{-1}(X)}^{2} \leq C_{1}^{\prime}\left(\|P u\|_{H_{0, \mathrm{~b}, \mathrm{loc}}^{-1, k}(X)}^{2}+\|\tilde{G} P u\|_{H_{0}^{-1}(X)}^{2}\right) .
$$

Combining these gives, with $C_{0}^{\prime}=C_{1}+C_{1}^{\prime}$, the desired result:

$$
\begin{aligned}
\left|\left\langle\Lambda^{-} A_{r} P u, \Lambda^{*} A_{r} u\right\rangle\right| & \leq\left\|\Lambda^{-} A_{r} P u\right\|\left\|\Lambda^{*} A_{r} u\right\| \leq\left\|\Lambda^{-} A_{r} P u\right\|^{2}+\left\|\Lambda^{*} A_{r} u\right\|^{2} \\
& \leq C_{0}^{\prime}\left(\|u\|_{H_{0, \mathrm{~b}, \mathrm{loc}}^{1, k}(X)}^{2}+\|G u\|_{H_{0}^{1}(X)}^{2}+\|P u\|_{H_{0, \mathrm{~b}, \mathrm{loc}}^{-1, k}(X)}^{2}+\|\tilde{G} P u\|_{H_{0}^{-1}(X)}^{2}\right) .
\end{aligned}
$$

A similar argument, using that $A_{r}$ is uniformly bounded in $\Psi_{\mathrm{bc}}^{s+1 / 2}(X)$ (in fact in $\Psi_{\mathrm{bc}}^{s}(X)$ ), and $E^{*} A_{r}$ is uniformly bounded in $\Psi_{\mathrm{bc}}^{s-1 / 2}(X)$ (in fact in $\Psi_{\mathrm{bc}}^{-\infty}(X)$ ), shows that $\left\langle A_{r} P u, E^{*} A_{r} u\right\rangle$ is uniformly bounded.

Now we turn to the second term in (7-2), whose uniform boundedness is a direct consequence of Lemma 5.4 and Corollary 5.15. Indeed, by Lemma 5.4, $\left[P, A_{r}\right]$ is a bounded family in $\operatorname{Diff}_{0}^{2} \Psi_{\text {bc }}^{s-1}(X)$; hence $A_{r}^{*}\left[P, A_{r}\right]$ is a bounded family in $\operatorname{Diff}_{0}^{2} \Psi_{\mathrm{bc}}^{2 s-1}(X)$. Then one can apply Corollary 5.15 to conclude that

$$
\left\langle A_{r}^{*}\left[P, A_{r}\right] u, u\right\rangle \leq C^{\prime}\left(\|u\|_{H_{0, \mathrm{~b}, \mathrm{loc}}^{1, k}(X)}^{2}+\|G u\|_{H^{1}(X)}^{2}\right) .
$$

A more precise version, in terms of requirements on $P u$, is the following. Here, as in Section 2, we fix a positive definite inner product on the fibers of ${ }^{0} T^{*} X$ (that is, a Riemannian 0-metric) to compute $\|d v\|_{L^{2}\left(X ;{ }^{0} T^{*} X\right)}^{2}$; since $v$ has support in a compact set below, the choice of the inner product is irrelevant. 
Lemma 7.5 (see [Vasy 2008c, Lemma 4.4]). Suppose that $K \subset{ }^{\mathrm{b}} S^{*} X$ is compact, $U \subset{ }^{\mathrm{b}} S^{*} X$ is open, and $K \subset U$. Suppose that $\mathscr{A}=\left\{A_{r}: r \in(0,1]\right\}$ is a bounded family of PsDOs in $\Psi_{\mathrm{bc}}^{s}(X)$ with $\mathrm{WF}_{\mathrm{b}}^{\prime}(\mathscr{A}) \subset K$ and with $A_{r} \in \Psi_{\mathrm{b}}^{s-1}(X)$ for $r \in(0,1]$. Then there are $G \in \Psi_{\mathrm{b}}^{s-1 / 2}(X)$ and $\tilde{G} \in \Psi_{\mathrm{b}}^{s}(X)$ with $\mathrm{WF}_{\mathrm{b}}^{\prime}(G), \mathrm{WF}_{\mathrm{b}}^{\prime}(\tilde{G}) \subset U$ and $C_{0}>0$ such that for $\epsilon>0, r \in(0,1], u \in H_{0, \mathrm{~b}, \mathrm{loc}}^{1, k}(X)$ (where $\left.k \leq 0\right)$ with neither $\mathrm{WF}_{\mathrm{b}}^{1, s-1 / 2}(u)$ nor $\mathrm{WF}_{\mathrm{b}}^{-1, s}(P u)$ intersecting $U$, we have

$$
\begin{aligned}
& \left|\left\langle d A_{r} u, d A_{r} u\right\rangle_{G}+\lambda\left\|A_{r} u\right\|^{2}\right| \\
& \quad \leq \epsilon\left\|d A_{r} u\right\|_{L^{2}\left(X ; T^{*} X\right)}^{2}+C_{0}\left(\|u\|_{H_{0, b, \mathrm{loc}}^{1, k}(X)}^{2}+\|G u\|_{H_{0}^{1}(X)}^{2}+\epsilon^{-1}\|P u\|_{H_{0, b, \mathrm{loc}}^{-1, k}(X)}^{2}+\epsilon^{-1}\|\tilde{G} P u\|_{H_{0}^{-1}(X)}^{2}\right) .
\end{aligned}
$$

Remark 7.6. The point of this lemma is that on the one hand the new term $\epsilon\left\|d A_{r} u\right\|^{2}$ can be absorbed in the left hand side in the elliptic region and hence is negligible; on the other hand, there is a gain in the order of $\tilde{G}$ ( $s$ versus $s+1 / 2$ in the previous lemma).

Proof. We need only modify the previous proof slightly, by estimating the term $\left|\left\langle A_{r} P u, A_{r} u\right\rangle\right|$ in (7-2) differently, namely

$$
\left|\left\langle A_{r} P u, A_{r} u\right\rangle\right| \leq\left\|A_{r} P u\right\|_{H_{0}^{-1}(X)}\left\|A_{r} u\right\|_{H_{0}^{1}(X)} \leq \tilde{\epsilon}\left\|A_{r} u\right\|_{H_{0}^{1}(X)}^{2}+\tilde{\epsilon}^{-1}\left\|A_{r} P u\right\|_{H_{0}^{-1}(X)}^{2} .
$$

Now the lemma follows by using Lemma 5.14 and the remark following it: Choosing any $\tilde{G} \in \Psi_{\mathrm{b}}^{s}(X)$ that is elliptic on $K$ gives a constant $C_{1}^{\prime}>0$ such that

$$
\left\|A_{r} P u\right\|_{H_{0}^{-1}(X)}^{2} \leq C_{1}^{\prime}\left(\|P u\|_{H_{0, b, l o c}^{-1, k}(X)}^{2}+\|\tilde{G} P u\|_{H_{0}^{-1}(X)}^{2}\right) .
$$

We then use the Poincaré inequality to estimate $\left\|A_{r} u\right\|_{H_{0}^{1}(X)}$ by $C_{2}\left\|d A_{r} u\right\|_{L^{2}(X)}$, and finish the proof exactly as for Lemma 7.3.

We next state microlocal elliptic regularity. For this result the restrictions on $\lambda \in \mathbb{C}$ are weak (only a half-line is disallowed), but on the other hand, a solution $u$ satisfying our hypotheses may not exist for values of $\lambda$ when $\lambda \notin\left(-\infty,(n-1)^{2} / 4\right)$.

Proposition 7.7 (microlocal elliptic regularity). Suppose that $P=\square+\lambda, \lambda \in \mathbb{C} \backslash\left[(n-1)^{2} / 4, \infty\right)$ and $m \in \mathbb{R}$ or $m=\infty$. Suppose $u \in H_{0, \mathrm{~b}, \mathrm{loc}}^{1, k}(X)$ for some $k \leq 0$. Then

$$
\mathrm{WF}_{\mathrm{b}}^{1, m}(u) \backslash \dot{\Sigma} \subset \mathrm{WF}_{\mathrm{b}}^{-1, m}(P u) .
$$

Proof. We first prove a slightly weaker result in which $\mathrm{WF}_{\mathrm{b}}^{-1, m}(P u)$ is replaced by $\mathrm{WF}_{\mathrm{b}}^{-1, m+1 / 2}(P u)-$ we rely on Lemma 7.3. We then prove the original statement using Lemma 7.5.

Suppose that $q \in{ }^{\mathrm{b}} T_{Y}^{*} X \backslash \dot{\Sigma}$. We may assume iteratively that $q \notin \mathrm{WF}_{\mathrm{b}}^{1, s-1 / 2}(u)$; we need to prove then that $q \notin \mathrm{WF}_{\mathrm{b}}^{1, s}(u)$ provided $s \leq m+1 / 2$ (note that the inductive hypothesis holds for $s=k+1 / 2$ since $\left.u \in H_{0, \mathrm{~b}, \mathrm{loc}}^{1, k}(X)\right)$. We use local coordinates $(x, y)$ as in Section 6 , centered so that $q \in{ }^{\mathrm{b}} T_{(0,0)}^{*} X$ and arranging that (6-7) holds. We further group the variables as $y=\left(y^{\prime}, y_{n-1}\right)$, with corresponding b-dual variables $\left(\underline{\zeta}^{\prime}, \underline{\zeta}_{n-1}\right)$. We denote the Euclidean norm by $\left|\underline{\zeta^{\prime}}\right|$.

Let $A \in \Psi_{\mathrm{b}}^{s}(X)$ be such that

$$
\mathrm{WF}_{\mathrm{b}}^{\prime}(A) \cap \mathrm{WF}_{\mathrm{b}}^{1, s-1 / 2}(u)=\varnothing \quad \text { and } \quad \mathrm{WF}_{\mathrm{b}}^{\prime}(A) \cap \mathrm{WF}_{\mathrm{b}}^{1, s+1 / 2}(P u)=\varnothing
$$


and that $\mathrm{WF}_{\mathrm{b}}^{\prime}(A)$ in a small conic neighborhood $U$ of $q$, with $U$ such that for a suitable $C>0$ or $\epsilon>0$,

(i) $\underline{\zeta}_{n-1}^{2}<C \underline{\xi}^{2}$ if $\underline{\xi}(q) \neq 0$,

(ii) $|\underline{\xi}|<\epsilon|\underline{\zeta}|$ for all $j$, and $\left|\underline{\zeta}^{\prime}\right| /\left|\underline{\zeta}_{n-1}\right|>1+\epsilon$ if $\underline{\xi}(q)=0$ and $\underline{\zeta}(q) \cdot B(y(q)) \underline{\zeta}(q)<0$.

Let $\Lambda_{r} \in \Psi_{\mathrm{b}}^{-2}(X)$ for $r>0$, such that $\mathscr{L}=\left\{\Lambda_{r}: r \in(0,1]\right\}$ is a bounded family in $\Psi_{\mathrm{b}}^{0}(X)$, and $\Lambda_{r} \rightarrow \operatorname{Id}$ as $r \rightarrow 0$ in $\Psi_{\mathrm{b}}^{\tilde{\epsilon}}(X)$ for $\tilde{\epsilon}>0$. For example, the symbol of $\Lambda_{r}$ could be taken as $\left(1+r\left(|\zeta|^{2}+|\underline{\xi}|^{2}\right)\right)^{-1}$. Let $A_{r}=\Lambda_{r} A$. Let $a$ be the symbol of $A$, and let $A_{r}$ have symbol $\left(1+r\left(|\zeta|^{2}+|\underline{\xi}|^{2}\right)\right)^{-1} \bar{a}$ for $r>0$, so $A_{r} \in \Psi_{\mathrm{b}}^{s-2}(X)$ for $r>0$, and $A_{r}$ is uniformly bounded in $\Psi_{\mathrm{bc}}^{s}(X)$, and $A_{r} \rightarrow A$ in $\Psi_{\mathrm{bc}}^{s+\tilde{\epsilon}}(X)$.

By Lemma 7.3,

$$
\left\langle d A_{r} u, d A_{r} u\right\rangle_{G}+\lambda\left\|A_{r} u\right\|^{2}
$$

is uniformly bounded for $r \in(0,1]$, so

$$
\left\langle d A_{r} u, d A_{r} u\right\rangle_{G}+\operatorname{Re} \lambda\left\|A_{r} u\right\|^{2} \quad \text { and } \quad \operatorname{Im} \lambda\left\|A_{r} u\right\|^{2}
$$

are uniformly bounded. If $\operatorname{Im} \lambda \neq 0$, then taking the imaginary part at once shows that $\left\|A_{r} u\right\|$ is in fact uniformly bounded. On the other hand, whether $\operatorname{Im} \lambda=0$ or not,

$$
\begin{array}{rl}
\left\langle d A_{r} u, d A_{r} u\right\rangle_{G}=\int_{X} & A(x, y) x D_{x} A_{r} u \overline{x D_{x} A_{r} u} d g+\int_{X} \sum B_{i j}(x, y) x D_{y_{i}} A_{r} u \overline{x D_{y_{j}} A_{r} u} d g \\
& +\int_{X} \sum C_{j}(x, y) x D_{x} A_{r} u \overline{x D_{y_{j}} A_{r} u} d g+\int_{X} \sum C_{j}(x, y) x D_{y_{j}} A_{r} u \overline{x D_{x} A_{r} u} d g .
\end{array}
$$

Using that $A(x, y)=-1+x A^{\prime}(x, y)+\sum\left(y_{j}-y_{j}(q)\right) A_{j}(x, y)$, we see that if $A_{r}$ is supported where $x<\delta$ and $\left|y_{j}-y_{j}(q)\right|<\delta$ for all $j$, then for some $C>0$ (independent of $A_{r}$ ),

$$
\left|\int_{X} A(x, y) x D_{x} A_{r} u \overline{x D_{x} A_{r} u} d g-\int_{X} A(0, y(q)) x D_{x} A_{r} u \overline{x D_{x} A_{r} u} d g\right| \leq C \delta\left\|x D_{x} A_{r} u\right\|^{2},
$$

with analogous estimates ${ }^{4}$ for $B_{i j}(x, y)-B_{i j}(0, y(q))$ and for $C_{j}(x, y)$. Thus, there exists $\tilde{C}>0$ and $\delta_{0}>0$ such that if $\delta<\delta_{0}$ and $A$ is supported where $|x|<\delta$ and $|y-y(q)|<\delta$, then

$$
\begin{array}{r}
\int_{X}\left((1-\tilde{C} \delta)\left|x D_{x} A_{r} u\right|^{2}-\operatorname{Re} \lambda\left|A_{r} u\right|^{2}\right) d g+\sum_{j=1}^{n-2} \int_{X}\left((1-\tilde{C} \delta) \sum_{j} x D_{y_{j}} A_{r} u \overline{x D_{y_{j}} A_{r} u}\right) d g \\
-\int_{X}\left((1+\tilde{C} \delta) \sum_{j} x D_{y_{n-1}} A_{r} u \overline{x D_{y_{n-1}} A_{r} u}\right) d g \\
\leq\left|\left\langle d A_{r} u, d A_{r} u\right\rangle_{G}+\operatorname{Re} \lambda\left\|A_{r} u\right\|^{2}\right| .
\end{array}
$$

Now we distinguish the cases $\underline{\xi}(q)=0$ and $\underline{\xi}(q) \neq 0$. If $\underline{\xi}(q)=0$, we choose $\delta \in(0,1 /(2 \tilde{C}))$ with $\delta<\delta_{0}$, so that

$$
(1-\tilde{C} \delta)\left(\left|\underline{\zeta}^{\prime}\right|^{2} / \underline{\zeta}_{n-1}^{2}\right)>1+2 \tilde{C} \delta
$$

\footnotetext{
${ }^{4}$ Recall that $C_{j}(0, y)=0$ and $B_{i j}(0, y(q))=0$ if $i \neq j$ and $B_{i j}(0, y(q))=1$ if $i=j=n-1$ and $B_{i j}(0, y(q))=-1$ if $i=j \neq n-1$.
} 
on a neighborhood of $\mathrm{WF}_{\mathrm{b}}^{\prime}(A)$, which is possible in view of (ii) at the beginning of the proof. Then the second integral on the left side of (7-5) can be written as $\left\|B x A_{r} u\right\|^{2}$, with the symbol of $B$ given by

$$
\left((1-\tilde{C} \delta)\left|\underline{\zeta}^{\prime}\right|^{2}-(1+\tilde{C} \delta) \underline{\zeta}_{n-1}^{2}\right)^{1 / 2}
$$

(which is $\left.\geq \delta\left|\underline{\zeta}_{n-1}\right|\right)$, modulo a term

$$
\int_{X} F x A_{r} u \overline{x A_{r} u} d g \text { for } F \in \Psi_{\mathrm{b}}^{1}(X) .
$$

But $A_{r}^{*} x F x A_{r}$ is uniformly bounded in $x^{2} \Psi_{\mathrm{bc}}^{2 s+1}(X) \subset \operatorname{Diff}_{0}^{2} \Psi_{\mathrm{bc}}^{2 s-1}(X)$, so this expression is uniformly bounded as $r \rightarrow 0$ by Corollary 5.15. We thus deduce that

$$
\int_{X}\left((1-\tilde{C} \delta)\left|x D_{x} A_{r} u\right|^{2}-\operatorname{Re} \lambda\left|A_{r} u\right|^{2}\right) d g+\left\|B x A_{r} u\right\|^{2}
$$

is uniformly bounded as $r \rightarrow 0$.

If $\underline{\xi}(q) \neq 0$, and $A$ is supported in $|x|<\delta$, then

$$
\tilde{C} \delta \int_{X} \delta^{-2}\left|x^{2} D_{x} A_{r} u\right|^{2} d g \leq \tilde{C} \delta \int_{X}\left|x D_{x} A_{r} u\right|^{2} d g .
$$

On the other hand, near $\left\{q^{\prime}: \underline{\xi}\left(q^{\prime}\right)=0\right\}$, for $\delta>0$ sufficiently small,

$$
\int_{X}\left(\frac{\tilde{C} \delta}{\delta^{2}}\left|x^{2} D_{x} A_{r} u\right|^{2}-\left|x D_{y_{n-1}} A_{r} u\right|^{2}\right) d g=\left\|B x A_{r} u\right\|^{2}+\int_{X} F x A_{r} u \overline{x A_{r} u} d g
$$

with the symbol of $B$ given by $\left((\tilde{C} / \delta) \underline{\xi}^{2}-\underline{\zeta}_{n-1}^{2}\right)^{1 / 2}$ (which does not vanish on $U$ for $\delta>0$ small), while $F \in \Psi_{\mathrm{b}}^{1}(X)$, so the second term on the right side is uniformly bounded as $r \rightarrow 0$ just as above. We thus deduce in this case that

$$
\int_{X}\left((1-2 \tilde{C} \delta)\left|x D_{x} A_{r} u\right|^{2} d g-\operatorname{Re} \lambda\left|A_{r} u\right|^{2}\right)+\left\|B x A_{r} u\right\|^{2}
$$

is uniformly bounded as $r \rightarrow 0$.

If $\operatorname{Im} \lambda \neq 0$ then we already saw that $\left\|A_{r} u\right\|_{L^{2}}$ is uniformly bounded, so we deduce that

$$
A_{r} u, x D_{x} A_{r} u \text { and } B x A_{r} u \text { are uniformly bounded in } L^{2}(X) \text {. }
$$

If $\operatorname{Im} \lambda=0$ but $\lambda<(n-1)^{2} / 4$, then the Poincaré inequality allows us to reach the same conclusion, since on the one hand in case (ii)

$$
(1-\tilde{C} \delta)\left\|x D_{x} A_{r} u\right\|^{2}-\operatorname{Re} \lambda\left\|A_{r} u\right\|^{2},
$$

and in case (i)

$$
(1-2 \tilde{C} \delta)\left\|x D_{x} A_{r} u\right\|^{2}-\operatorname{Re} \lambda\left\|A_{r} u\right\|^{2},
$$


are uniformly bounded; on the other hand by Proposition 2.3, for $\delta>0$ sufficiently small there exists $c>0$ such that

$$
(1-2 \tilde{C} \delta)\left\|x D_{x} A_{r} u\right\|^{2}-\operatorname{Re} \lambda\left\|A_{r} u\right\|^{2} \geq c\left(\left\|x D_{x} A_{r} u\right\|^{2}+\left\|A_{r} u\right\|^{2}\right) .
$$

Correspondingly there are sequences $A_{r_{k}} u, x D_{x} A_{r_{k}} u$ and $B x A_{r_{k}} u$, weakly convergent in $L^{2}(X)$, and such that $r_{k} \rightarrow 0$, as $k \rightarrow \infty$. Since they respectively converge to $A u, x D_{x} A u$ and $B x A u$ in $\mathscr{C}^{-\infty}(X)$, we deduce that the weak limits are $A u, x D_{x} A u$ and $B x A u$, which therefore lie in $L^{2}(X)$. Consequently, $q \notin \mathrm{WF}_{\mathrm{b}}^{1, s}(u)$, hence proving the proposition with $\mathrm{WF}_{\mathrm{b}}^{-1, m}(P u)$ replaced by $\mathrm{WF}_{\mathrm{b}}^{-1, m+1 / 2}(P u)$.

To obtain the optimal result, we note that due to Lemma 7.5 we still have, for any $\epsilon>0$, that

$$
\left\langle d A_{r} u, d A_{r} u\right\rangle_{G}-\epsilon\left\|d A_{r} u\right\|^{2}
$$

is uniformly bounded above for $r \in(0,1]$. By arguing just as above, with $B$ as above, for sufficiently small $\epsilon>0$, the right side gives an upper bound for

$$
\int_{X}\left((1-2 \tilde{C} \delta-\epsilon)\left|x D_{x} A_{r} u\right|^{2}-\operatorname{Re} \lambda\left|A_{r} u\right|^{2}\right) d g+\left\|B x A_{r} u\right\|^{2},
$$

which is thus uniformly bounded as $r \rightarrow 0$. The proof is then finished exactly as above.

The analogous argument works for the conformally compact elliptic problem, that is, on asymptotically hyperbolic spaces, to give that for $\lambda \in \mathbb{C} \backslash\left[(n-1)^{2} / 4, \infty\right)$, local solutions of $\left(\Delta_{g}-\lambda\right) u$ are actually conormal to $Y$ provided they lie in $H_{0}^{1}(X)$ locally, or indeed in $H_{0, \mathrm{~b}}^{1,-\infty}(X)$.

\section{Propagation of singularities}

In this section we prove propagation of singularities for $P$ by positive commutator estimates. We do so by first performing a general commutator calculation in Proposition 8.1, then using it to prove rough propagation estimates first at hyperbolic, then at glancing points, in Propositions 8.2 and 8.6, respectively. An argument originally due to Melrose and Sjöstrand [1978] then proves the main theorems, Theorems 8.8 and 8.9. Finally we discuss consequences of these results.

We first describe the form of commutators of $P$ with $\Psi_{\mathrm{b}}(X)$. We state this as an analogue of [Vasy 2010a, Proposition 3.10], and later in the section we follow the structure of [Vasy 2010a] as well. Given Proposition 8.1 below, the proof of propagation of singularities proceeds with the same commutant construction as in [Vasy 2008c]; see also [Vasy 2008a]. Although it is in a setting that is more complicated in some ways, since it deals with the equation on differentials forms, we follow the structure of [Vasy 2010a] since it was written in a more systematic way than [Vasy 2008c]. Recall from the introduction that $\underline{\xi}$ is the variable b-dual to $x$, and $\underline{\hat{\xi}}=\underline{\xi} /\left|\underline{\zeta}_{n-1}\right|$.

Proposition 8.1. Suppose $\mathscr{A}=\left\{A_{r}: r \in(0,1]\right\}$ is a family of operators $A_{r} \in \Psi_{\mathrm{b}}^{0}(X)$ uniformly bounded in $\Psi_{\mathrm{bc}}^{s+1 / 2}(X)$, of the form $A_{r}=A \Lambda_{r}$, with $A \in \Psi_{\mathrm{b}}^{0}(X), a=\sigma_{\mathrm{b}, 0}(A)$ and $w_{r}=\sigma_{\mathrm{b}, s+1 / 2}\left(\Lambda_{r}\right)$. Then

$$
\imath\left[A_{r}^{*} A_{r}, \square\right]=\left(x D_{x}\right)^{*} C_{r}^{\sharp}\left(x D_{x}\right)+\left(x D_{x}\right)^{*} x C_{r}^{\prime}+x C_{r}^{\prime \prime}\left(x D_{x}\right)+x^{2} C_{r}^{b},
$$


where

$$
C_{r}^{\sharp} \in L^{\infty}\left((0,1] ; \Psi_{\mathrm{bc}}^{2 s}(X)\right), \quad C_{r}^{\prime}, C_{r}^{\prime \prime} \in L^{\infty}\left((0,1] ; \Psi_{\mathrm{bc}}^{2 s+1}(X)\right), \quad C_{r}^{\mathrm{b}} \in \Psi_{\mathrm{bc}}^{2 s+2}(X),
$$

and

$$
\begin{aligned}
\sigma_{\mathrm{b}, 2 s}\left(C_{r}^{\sharp}\right) & =2 w_{r}^{2} a\left(V^{\sharp} a+a \tilde{c}_{r}^{\sharp}\right), \\
\sigma_{\mathrm{b}, 2 s+1}\left(C_{r}^{\prime}\right) & =\sigma_{\mathrm{b}, 2 s+1}\left(C_{r}^{\prime \prime}\right)=2 w_{r}^{2} a\left(V^{\prime} a+a \tilde{c}_{r}^{\prime}\right), \\
\sigma_{\mathrm{b}, 2 s+2}\left(C_{r}^{\mathrm{b}}\right) & =2 w_{r}^{2} a\left(V^{\mathrm{b}} a+a \tilde{c}_{r}^{\mathrm{b}}\right),
\end{aligned}
$$

with $\tilde{c}_{r}^{\sharp}, \tilde{c}_{r}^{\prime}, \tilde{c}_{r}^{b}$ uniformly bounded in $S^{-1}, S^{0}, S^{1}$, respectively, $V^{\sharp}, V^{\prime}, V^{b}$ smooth and homogeneous of degree $-1,0,1$ respectively on ${ }^{\mathrm{b}} T^{*} X \backslash$ o, and where $\left.V^{\sharp}\right|_{Y}$ and $\left.V^{\prime}\right|_{Y}$ annihilate $\xi$ and

$$
\left.V^{b}\right|_{Y}=2 h \partial_{\underline{\xi}}-\mathrm{H}_{h}
$$

Proof. In Proposition 7.1, $\square$ is decomposed into a sum of products of weighted b-operators, so analogously expanding the commutator, all calculations can be done in $x^{l} \Psi_{\mathrm{b}}(X)$ for various values of $l$. In particular, keeping in mind Lemma 5.1 (which gives the additional order of decay),

$$
\imath\left[A_{r}^{*} A_{r}, x D_{x}\right], \imath\left[A_{r}^{*} A_{r},\left(x D_{x}\right)^{*}\right] \in L^{\infty}\left((0,1]_{r}, x \Psi_{\mathrm{b}}^{2 s+1}(X)\right),
$$

with principal symbol $-2 w_{r}^{2} a x \partial_{x} a-2 a^{2} w_{r}\left(x \partial_{x} w_{r}\right)$. By this observation, all commutators with factors of $x D_{x}$ or $\left(x D_{x}\right)^{*}$ in (7-1) can be absorbed into the "next term" of (8-1), so [ $\left.A_{r}^{*} A_{r},\left(x D_{x}\right)^{*}\right] \alpha\left(x D_{x}\right)$ is absorbed into $x C_{r}^{\prime \prime}\left(x D_{x}\right),\left(x D_{x}\right) \alpha\left[A_{r}^{*} A_{r}, x D_{x}\right]$ is absorbed into $\left(x D_{x}\right)^{*} x C_{r}^{\prime}$, and $\left[A_{r}^{*} A_{r},\left(x D_{x}\right)^{*}\right] M^{\prime}$ and $M^{\prime \prime}\left[A_{r}^{*} A_{r},\left(x D_{x}\right)\right]$ are absorbed into $x^{2} C_{r}^{b}$. The principal symbols of these terms are of the desired form, that is, after factoring out $2 w_{r}^{2} a$, they are the result of a vector field applied to $a$ plus a multiple of $a$, and this vector field is $-\alpha \partial_{x}$ in the case of the first two terms (thus annihilating $\xi$ ), and is $-m x^{-1} \partial_{x}$ in the case of the last two terms, which in view of $m=\sigma_{\mathrm{b}, 1}\left(M^{\prime}\right)=\sigma_{\mathrm{b}, 1}\left(M^{\prime \prime}\right) \in x^{2} S^{1}$, shows that it actually does not affect $\left.V^{b}\right|_{Y}$.

Next, $\iota\left(x D_{x}\right)^{*}\left[A_{r}^{*} A_{r}, \alpha\right]\left(x D_{x}\right)$ can be absorbed into (and can be taken equal to) $\left(x D_{x}\right)^{*} C_{r}^{\sharp}\left(x D_{x}\right)$ with principal symbol of $C_{r}^{\sharp}$ given by

$$
-\left(\partial_{y} \alpha\right) \partial \underline{\zeta}\left(a^{2} w_{r}^{2}\right)-\left(x \partial_{x} \alpha\right) \partial_{\underline{\xi}}\left(a^{2} w_{r}^{2}\right)
$$

in local coordinates; thus again is of the desired form since the $\partial_{\underline{\xi}}$ term has a vanishing factor of $x$ preceding it.

Since $\left[A_{r}^{*} A_{r}, M^{\prime}\right]$ and $\left[A_{r}^{*} A_{r}, M^{\prime \prime}\right]$ are uniformly bounded in $x^{2} \Psi_{\mathrm{b}}^{2 s+1}(X)$, the corresponding commutators can be absorbed into $\left(x D_{x}\right)^{*} x C_{r}^{\prime}$ and $x C_{r}^{\prime \prime}\left(x D_{x}\right)$, respectively, without affecting the principal symbols of $C_{r}^{\prime}$ and $C_{r}^{\prime \prime}$ at $Y$, and possessing the desired form.

Next, $\tilde{P}=x^{2} \square_{h}+R$, with $R \in x^{3} \operatorname{Diff}_{\mathrm{b}}^{2}(X)$, so $\left[A_{r}^{*} A_{r}, R\right]$ is uniformly bounded in $x^{3} \Psi_{\mathrm{b}}^{2 s+2}(X)$, and thus can be absorbed into $C_{r}^{b}$ without affecting its principal symbol at $Y$, and it has the desired form. Finally, $\imath\left[A_{r}^{*} A_{r}, x^{2} \square_{h}\right] \in x^{2} \Psi_{\mathrm{b}}^{2 s+2}(X)$ has principal symbol $\partial_{\underline{\xi}}\left(a^{2} w_{r}^{2}\right) 2 x^{2} h-x^{2} \mathrm{H}_{h}\left(a^{2} w_{r}^{2}\right)$, and can thus be absorbed into $C_{r}^{b}$, yielding the stated principal symbol at $\bar{Y}$.

We start our propagation results with the propagation estimate at hyperbolic points. 
Proposition 8.2 (normal, or hyperbolic, propagation). Suppose $P=\square_{g}+\lambda$, with $\lambda \in \mathbb{C} \backslash\left[(n-1)^{2} / 4, \infty\right)$. Let $q_{0}=\left(0, y_{0}, 0, \underline{\zeta}_{0}\right) \in \mathscr{H}^{\mathrm{b}} T_{Y}^{*} X$, and let

$$
\eta=-\underline{\hat{\xi}}
$$

be the function defined in the local coordinates discussed above, and suppose that $u \in H_{0, \mathrm{~b}, \mathrm{loc}}^{1, k}(X)$ for some $k \leq 0, q_{0} \notin \mathrm{WF}_{\mathrm{b}}^{-1, \infty}(f)$ and $f=P u$. If $\operatorname{Im} \lambda \leq 0$ and there exists a conic neighborhood $U$ of $q_{0}$ in ${ }^{\mathrm{b}} T^{*} X \backslash$ o such that

$$
q \notin \mathrm{WF}_{\mathrm{b}}^{1, \infty}(u) \text { if } q \in U \text { and } \eta(q)<0,
$$

then $q_{0} \notin \mathrm{WF}_{\mathrm{b}}^{1, \infty}(u)$.

In fact, if the wave front set assumptions are relaxed to $q_{0} \notin \mathrm{WF}_{\mathrm{b}}^{-1, s+1}(f)$ (with $f=P u$ ) and the existence of a conic neighborhood $U$ of $q_{0}$ in ${ }^{\mathrm{b}} T^{*} X \backslash$ o such that

$$
q \notin \mathrm{WF}_{\mathrm{b}}^{1, s}(u) \quad \text { if } q \in U \text { and } \eta(q)<0,
$$

then we can still conclude that $q_{0} \notin \mathrm{WF}_{\mathrm{b}}^{1, s}(u)$.

Remark 8.3. As follows immediately from the proof given below, in (8-3) and (8-4), one can replace $\eta(q)<0$ by $\eta(q)>0$, that is, one has the conclusion for either direction (backward or forward) of propagation, provided one also switches the sign of $\operatorname{Im} \lambda$ when it is nonzero that is, the assumption should be $\operatorname{Im} \lambda \geq 0$. In particular, if $\operatorname{Im} \lambda=0$, one obtains propagation estimates both along increasing and along decreasing $\eta$.

Note that $\eta$ is increasing along the GBB of $\square_{\hat{g}}$ by (6-11). Thus, the hypothesis region $\{q \in U: \eta(q)<0\}$ on the left side of (8-3) is backwards from $q_{0}$, so this proposition, roughly speaking, propagates regularity forwards.

Moreover, every neighborhood $U$ of $q_{0}=\left(y_{0}, \underline{\zeta}_{0}\right) \in \mathscr{H} \cap{ }^{\mathrm{b}} T_{Y}^{*} X$ in $\dot{\Sigma}$ contains an open set of the form

$$
\left\{q:|x(q)|^{2}+\left|y(q)-y_{0}\right|^{2}+\left|\underline{\hat{\zeta}}(q)-\underline{\zeta}_{0}\right|^{2}<\delta\right\},
$$

see [Vasy 2008c, Equation (5.1)]. Note also that (8-3) implies the same statement with $U$ replaced by any smaller neighborhood of $q_{0}$ and in particular for the set (8-5), provided that $\delta$ is sufficiently small. We can also assume by the same observation that $\mathrm{WF}_{\mathrm{b}}^{-1, s+1}(P u) \cap U=\varnothing$. Furthermore, we can also arrange that $h(x, y, \underline{\xi}, \underline{\zeta})>|(\underline{\xi}, \underline{\zeta})|^{2}\left|\underline{\zeta}_{0}\right|^{-2} h\left(q_{0}\right) / 2$ on $U$ since $\underline{\zeta}_{0} \cdot B\left(y_{0}\right) \underline{\zeta}_{0}=h\left(0, y_{0}, 0, \underline{\zeta}_{0}\right)>0$. We write

$$
\hat{h}=\left|\underline{\zeta}_{n-1}\right|^{-2} h=\left|\underline{\zeta}_{n-1}\right|^{-2} \underline{\zeta} \cdot B(y) \underline{\zeta}
$$

for the rehomogenized version of $h$, which is thus homogeneous of degree zero and bounded below by a positive constant on $U$.

Proof. This proposition is the analogue of [Vasy 2008c, Proposition 6.2], and since the argument is similar, we mainly emphasize the differences. These enter by virtue of $\lambda$ not being negligible and the use of the Poincaré inequality. In [Vasy 2008c], one uses a commutant $A \in \Psi_{\mathrm{b}}^{0}(X)$ and weights $\Lambda_{r} \in$ $\Psi_{\mathrm{b}}^{0}(X)$ for $r \in(0,1)$, which are uniformly bounded in $\Psi_{\mathrm{bc}}^{s+1 / 2}(X)$, with $A_{r}=A \Lambda_{r}$, in order to obtain the propagation of $\mathrm{WF}_{\mathrm{b}}^{1, s}(u)$ with the notation of that paper, whose analogue is $\mathrm{WF}_{\mathrm{b}}^{1, s}(u)$ here (the 
difference is the space relative to which one obtains b-regularity: $H^{1}(X)$ in the previous paper, the zero-Sobolev space $H_{0}^{1}(X)$ here). One can use exactly the same commutant as in [Vasy 2008c]. Then Proposition 8.1 lets one calculate $\imath\left[A_{r}^{*} A_{r}, P\right]$ to obtain an expression completely analogous to [Vasy 2008c, Equation (6.18)] in the hyperbolic case. We also refer to [Vasy 2010a] because, although it studies a more delicate problem, namely natural boundary conditions (which are not scalar), the main ingredient of the proof, the commutator calculation, is written up exactly as above in Proposition 8.1; see [Vasy 2010a, Proposition 3.10] and the way it is used subsequently in Proposition 5.1 there.

As in the proof of [Vasy 2010a, Proposition 5.1], we first construct a commutant by defining its scalar principal symbol $a$. This completely follows the scalar case; see the proof [Vasy 2008c, Proposition 6.2]. Next we show how to obtain the desired estimate.

So, as in the proof [Vasy 2008c, Proposition 6.2], let

$$
\omega(q)=|x(q)|^{2}+\left|y(q)-y_{0}\right|^{2}+\left|\underline{\hat{\zeta}}(q)-\hat{\zeta}_{0}\right|^{2},
$$

with $|\cdot|$ denoting the Euclidean norm. For $\epsilon>0$ and $\delta>0$, with other restrictions to be imposed later on, let

$$
\phi=\eta+\frac{1}{\epsilon^{2} \delta} \omega,
$$

Let $\chi_{0} \in \mathscr{C}^{\infty}(\mathbb{R})$ be equal to 0 on $(-\infty, 0]$ and $\chi_{0}(t)=\exp (-1 / t)$ for $t>0$. Thus, $t^{2} \chi_{0}^{\prime}(t)=\chi_{0}(t)$ for $t \in \mathbb{R}$. Let $\chi_{1} \in \mathscr{C}^{\infty}(\mathbb{R})$ be 0 on $(-\infty, 0]$ and 1 on $[1, \infty)$, with $\chi_{1}^{\prime} \geq 0$ satisfying $\chi_{1}^{\prime} \in \mathscr{C}_{\text {comp }}^{\infty}((0,1))$. Finally, let $\chi_{2} \in \mathscr{C}_{\text {comp }}^{\infty}(\mathbb{R})$ be supported in $\left[-2 c_{1}, 2 c_{1}\right]$ and identically 1 on $\left[-c_{1}, c_{1}\right]$, where $c_{1}$ satisfies $|\underline{\xi}|^{2}<c_{1} / 2$ in $\dot{\Sigma} \cap U$. Thus, $\chi_{2}\left(|\underline{\xi}|^{2}\right)$ is a cutoff in $|\hat{\xi}|$, with its support properties ensuring that $d \chi_{2}\left(|\hat{\xi}|^{2}\right)$ is supported in $|\hat{\xi}|^{2} \in\left[c_{1}, 2 c_{1}\right]$ and hence outside $\dot{\Sigma}$ - it should be thought of as a factor that microlocalizes near the characteristic set but effectively commutes with $P$ (since we already have the microlocal elliptic result). Then, for $\digamma>0$ large, to be determined, let

$$
a=\chi_{0}\left(\digamma^{-1}(2-\phi / \delta)\right) \chi_{1}(\eta / \delta+2) \chi_{2}\left(|\underline{\xi}|^{2}\right) ;
$$

so $a$ is a homogeneous degree zero $\mathscr{C}^{\infty}$ function on a conic neighborhood of $q_{0}$ in ${ }^{\mathrm{b}} T^{*} X \backslash o$. Indeed as we will see momentarily, $a$ has for any $\epsilon>0$ compact support inside this neighborhood (regarded as a subset of ${ }^{\mathrm{b}} S^{*} X$, that is, quotienting out by the $\mathbb{R}^{+}$-action) for $\delta$ sufficiently small, so in fact it is globally well defined. In fact, on supp $a$ we have $\phi \leq 2 \delta$ and $\eta \geq-2 \delta$. Since $\omega \geq 0$, the first of these inequalities implies that $\eta \leq 2 \delta$, so on supp $a$

$$
|\eta| \leq 2 \delta
$$

Hence,

$$
\omega \leq \epsilon^{2} \delta(2 \delta-\eta) \leq 4 \delta^{2} \epsilon^{2} .
$$

In view of (8-6) and (8-5), this shows that given any $\epsilon_{0}>0$ there exists $\delta_{0}>0$ such that $a$ is supported in $U$ for any $\epsilon \in\left(0, \epsilon_{0}\right)$ and $\delta \in\left(0, \delta_{0}\right)$. The role that $\digamma$ large plays (in the definition of $a$ ) is that it increases the size of the first derivatives of $a$ relative to the size of $a$; hence it allows us to give a bound for $a$ in terms of a small multiple of its derivative along the Hamilton vector field, much like the stress 
energy tensor was used to bound other terms by making $\chi^{\prime}$ large relative to $\chi$ in the (nonmicrolocal) energy estimate.

Now let $A_{0} \in \Psi_{\mathrm{b}}^{0}(X)$ with $\sigma_{\mathrm{b}, 0}\left(A_{0}\right)=a$, supported in the coordinate chart. Also let $\Lambda_{r}$ be scalar and have symbol

$$
\left|\underline{\zeta}_{n-1}\right|^{s+1 / 2}\left(1+r\left|\underline{\zeta}_{n-1}\right|^{2}\right)^{-s} \mathrm{Id} \quad \text { for } r \in[0,1),
$$

so $A_{r}=A \Lambda_{r} \in \Psi_{\mathrm{b}}^{0}(X)$ for $r>0$ and it is uniformly bounded in $\Psi_{\mathrm{bc}}^{s+1 / 2}(X)$. Then, for $r>0$,

$$
\begin{aligned}
\left\langle l A_{r}^{*} A_{r} P u, u\right\rangle-\left\langle\imath A_{r}^{*} A_{r} u, P u\right\rangle & =\left\langle\imath\left[A_{r}^{*} A_{r}, P\right] u, u\right\rangle+\left\langle l\left(P-P^{*}\right) A_{r}^{*} A_{r} u, u\right\rangle \\
& =\left\langle\imath\left[A_{r}^{*} A_{r}, P\right] u, u\right\rangle-2 \operatorname{Im} \lambda\left\|A_{r} u\right\|^{2} .
\end{aligned}
$$

We can compute this using Proposition 8.1. We arrange the terms of the proposition so that the terms in which a vector field differentiates $\chi_{1}$ and $\chi_{2}$ are included in $E_{r}$ and $E_{r}^{\prime}$, respectively. Thus, we have

$$
\imath A_{r}^{*} A_{r} P-{ }_{\imath} P A_{r}^{*} A_{r}=\left(x D_{x}\right)^{*} C_{r}^{\sharp}\left(x D_{x}\right)+\left(x D_{x}\right)^{*} x C_{r}^{\prime}+x C_{r}^{\prime \prime}\left(x D_{x}\right)+x^{2} C_{r}^{b}+E_{r}+E_{r}^{\prime}+F_{r},
$$

with

$$
\begin{aligned}
\sigma_{\mathrm{b}, 2 s}\left(C_{r}^{\sharp}\right) & =w_{r}^{2}\left(\digamma^{-1} \delta^{-1} a\left|\underline{\zeta}_{n-1}\right|^{-1}\left(\hat{f}^{\sharp}+\epsilon^{-2} \delta^{-1} f^{\sharp}\right) \chi_{0}^{\prime} \chi_{1} \chi_{2}+a^{2} \tilde{c}_{r}^{\sharp}\right), \\
\sigma_{\mathrm{b}, 2 s+1}\left(C_{r}^{\prime}\right) & =w_{r}^{2}\left(\digamma^{-1} \delta^{-1} a\left(\hat{f}^{\prime}+\delta^{-1} \epsilon^{-2} f^{\prime}\right) \chi_{0}^{\prime} \chi_{1} \chi_{2}+a^{2} \tilde{c}_{r}^{\prime}\right), \\
\sigma_{\mathrm{b}, 2 s+1}\left(C_{r}^{\prime \prime}\right) & =w_{r}^{2}\left(\digamma^{-1} \delta^{-1} a\left(\hat{f}^{\prime \prime}+\delta^{-1} \epsilon^{-2} f^{\prime \prime}\right) \chi_{0}^{\prime} \chi_{1} \chi_{2}+a^{2} \tilde{c}_{r}^{\prime \prime}\right), \\
\sigma_{\mathrm{b}, 2 s+2}\left(C_{r}\right) & =w_{r}^{2}\left(\digamma^{-1} \delta^{-1}\left|\underline{\zeta}_{n-1}\right| a\left(4 \hat{h}+\hat{f}^{b}+\delta^{-1} \epsilon^{-2} f^{\mathrm{b}}\right) \chi_{0}^{\prime} \chi_{1} \chi_{2}+a^{2} \tilde{c}_{r}^{b}\right),
\end{aligned}
$$

where $f^{\sharp}, f^{\prime}, f^{\prime \prime}$ and $f^{\mathrm{b}}$ as well as $\hat{f}^{\sharp}, \hat{f}^{\prime}, \hat{f}^{\prime \prime}$ and $\hat{f}^{\mathrm{b}}$ are all smooth functions on ${ }^{\mathrm{b}} T^{*} X \backslash o$, homogeneous of degree 0 (independent of $\epsilon$ and $\delta$ ), and $\hat{h}=\left|\zeta_{n-1}\right|^{-2} h$ is the rehomogenized version of $h$. Moreover, $f^{\sharp}, f^{\prime}, f^{\prime \prime}$ and $f^{b}$ arise from when $\omega$ is differentiated in $\chi\left(\digamma^{-1}(2-\phi / \delta)\right)$, and thus vanish when $\omega=0$, while $\hat{f}^{\sharp}, \hat{f}^{\prime}, \hat{f}^{\prime \prime}$ and $\hat{f}^{b}$ arise when $\eta$ is differentiated in $\chi\left(\digamma^{-1}(2-\phi / \delta)\right)$, and comprise all such terms with the exception of those arising from the $\partial_{\xi}$ component of $\left.V^{\mathrm{b}}\right|_{Y}$ (which gives $4 \hat{h}=4\left|\zeta_{n-1}\right|^{-2} h$ on the last line above) and hence are the sums of functions vanishing at $x=0$ (corresponding to us only specifying the restrictions of the vector fields in (8-2) at $Y$ ) and functions vanishing at $\hat{\xi}=0$ (when $\left|\underline{\zeta}_{n-1}\right|^{-1}$ in $\eta=-\underline{\xi}\left|\underline{\zeta}_{n-1}\right|^{-1}$ is differentiated). ${ }^{5}$

In this formula we think of

$$
4 \digamma^{-1} \delta^{-1} w_{r}^{2} a\left|\underline{\zeta}_{n-1}\right| \hat{h} \chi_{0}^{\prime} \chi_{1} \chi_{2}
$$

as the main term; note that $\hat{h}$ is positive near $q_{0}$. Compared to this, the terms with $a^{2}$ are negligible, for they can all be bounded by

$$
c \digamma^{-1}\left(\digamma^{-1} \delta^{-1} w_{r}^{2} a\left|\underline{\zeta}_{n-1}\right|^{-1} \chi_{0}^{\prime} \chi_{1} \chi_{2}\right)
$$

(see (8-15)), that is, by a small multiple of $\digamma^{-1} \delta^{-1} w_{r}^{2} a\left|\underline{\zeta}_{n-1}\right|^{-1} \chi_{0}^{\prime} \chi_{1} \chi_{2}$ when $\digamma$ is taken large, using that $2-\phi / \delta \leq 4$ on supp $a$ and

$$
\chi_{0}\left(\digamma^{-1} t\right)=\left(\digamma^{-1} t\right)^{2} \chi_{0}^{\prime}\left(\digamma^{-1} t\right) \leq 16 \digamma^{-2} \chi_{0}^{\prime}\left(\digamma^{-1} t\right) \text { for } t \leq 4 ;
$$

${ }^{5}$ Terms of the latter kind did not occur in [Vasy 2008c] since time-translation invariance was assumed, but it does occur in [Vasy 2008b] and [Vasy 2010a], where the Lorentzian scalar setting is considered. 
see the discussion in [Vasy 2008b, Section 6] and following [Vasy 2008c, Equation (6.19)].

The vanishing condition on the $f^{\sharp}, f^{\prime}, f^{\prime \prime}, f^{b}$ ensures that, on $\operatorname{supp} a$,

$$
\left|f^{\sharp}\right|,\left|f^{\prime}\right|,\left|f^{\prime \prime}\right|,\left|f^{b}\right| \leq C \omega^{1 / 2} \leq 2 C \epsilon \delta,
$$

so the corresponding terms can thus be estimated using $w_{r}^{2} \digamma^{-1} \delta^{-1} a\left|\zeta_{n-1}\right|^{-1} \chi_{0}^{\prime} \chi_{1} \chi_{2}$ provided $\epsilon^{-1}$ is not too large; that is, there exists $\tilde{\epsilon}_{0}>0$ such that if $\epsilon>\tilde{\epsilon}_{0}$, the terms with $f^{\sharp}, f^{\prime}, f^{\prime \prime}, f^{b}$ can be treated as error terms.

On the other hand, we have

$$
\left|\hat{f}^{\sharp}\right|,\left|\hat{f}^{\prime}\right|,\left|\hat{f}^{\prime \prime}\right|,\left|\hat{f}^{b}\right| \leq C|x|+C|\underline{\hat{\xi}}| \leq C \omega^{1 / 2}+C|\underline{\hat{\xi}}| \leq 2 C \epsilon \delta+C|\underline{\hat{\xi}}| .
$$

Now, $|\hat{\xi}| \leq 2|x|$ on $\dot{\Sigma}$ (for $|\xi|=x|\xi| \leq 2|x|\left|\zeta_{n-1}\right|$ with $U$ sufficiently small). Therefore we can write $\hat{f}^{\sharp}=\hat{f}_{\sharp}^{\sharp}+\hat{f}_{b}^{\sharp}$ with $\hat{f}_{b}^{\sharp}$ supported away from $\dot{\Sigma}$ and $\hat{f}_{\sharp}^{\sharp}$ satisfying

$$
\left|\hat{f}_{\sharp}^{\sharp}\right| \leq C|x|+C|\underline{\hat{\xi}}| \leq C^{\prime}|x| \leq C^{\prime} \omega^{1 / 2} \leq 2 C^{\prime} \epsilon \delta ;
$$

we can also obtain a similar decomposition for $\hat{f}^{\prime}, \hat{f}^{\prime \prime}$ and $\hat{f}^{b}$.

Indeed, using (8-16) it is useful to rewrite (8-14) as

$$
\begin{aligned}
\sigma_{\mathrm{b}, 2 s}\left(C_{r}^{\sharp}\right) & =w_{r}^{2} \digamma^{-1} \delta^{-1} a\left|\underline{\zeta}_{n-1}\right|^{-1}\left(\hat{f}^{\sharp}+\epsilon^{-2} \delta^{-1} f^{\sharp}+\digamma^{-1} \delta \hat{c}_{r}^{\sharp}\right) \chi_{0}^{\prime} \chi_{1} \chi_{2}, \\
\sigma_{\mathrm{b}, 2 s+1}\left(C_{r}^{\prime}\right) & =w_{r}^{2} \delta^{-1} \digamma^{-1} a\left(\hat{f}^{\prime}+\delta^{-1} \epsilon^{-2} f^{\prime}+\digamma^{-1} \delta \hat{c}_{r}^{\prime}\right) \chi_{0}^{\prime} \chi_{1} \chi_{2}, \\
\sigma_{\mathrm{b}, 2 s+1}\left(C_{r}^{\prime \prime}\right) & =w_{r}^{2} \delta^{-1} \digamma^{-1} a\left(\hat{f}^{\prime \prime}+\delta^{-1} \epsilon^{-2} f^{\prime \prime}+\digamma^{-1} \delta \hat{c}_{r}^{\prime \prime}\right) \chi_{0}^{\prime} \chi_{1} \chi_{2}, \\
\sigma_{\mathrm{b}, 2 s+2}\left(C_{r}^{b}\right) & =w_{r}^{2} \delta^{-1} \digamma^{-1} a\left|\underline{\zeta}_{n-1}\right|\left(4 \hat{h}+\hat{f}^{b}+\delta^{-1} \epsilon^{-2} f^{\mathrm{b}}+\digamma^{-1} \hat{c}_{r}^{b}\right) \chi_{0}^{\prime} \chi_{1} \chi_{2},
\end{aligned}
$$

where

- $f^{\sharp}, f^{\prime}, f^{\prime \prime}$ and $f^{\mathrm{b}}$ are smooth functions on ${ }^{\mathrm{b}} T^{*} X \backslash o$ that are homogeneous of degree 0 and satisfy (8-17) (and are independent of $\digamma, \epsilon, \delta, r$ );

- $\hat{f}^{\sharp}, \hat{f}^{\prime}, \hat{f}^{\prime \prime}$ and $\hat{f}^{\mathrm{b}}$ are smooth functions on ${ }^{\mathrm{b}} T^{*} X \backslash \rho$, homogeneous of degree 0 , with $\hat{f}^{\sharp}=\hat{f}_{\sharp}^{\sharp}+\hat{f}_{\mathrm{b}}^{\sharp}$, where $\hat{f}_{\sharp}^{\sharp}, \hat{f}_{\sharp}^{\prime}, \hat{f}_{\sharp}^{\prime \prime}, \hat{f}_{\sharp}^{b}$ satisfy (8-19) (and are independent of $\digamma, \epsilon, \delta, r$ ), while $\hat{f}_{b}^{\sharp}, \hat{f}_{b}^{\prime}, \hat{f}_{b}^{\prime \prime}, \hat{f}_{b}^{b}$ are supported away from $\dot{\Sigma}$; and

- $\hat{c}_{r}^{\sharp}, \hat{c}_{r}^{\prime}, \hat{c}_{r}^{\prime \prime}$ and $\hat{c}_{r}^{b}$ are smooth functions on ${ }^{\mathrm{b}} T^{*} X \backslash o$ that are homogeneous of degree 0 and uniformly bounded in $\epsilon, \delta, r, \digamma$.

Let

$$
b_{r}=2 w_{r}\left|\underline{\zeta}_{n-1}\right|^{1 / 2}(\digamma \delta)^{-1 / 2}\left(\chi_{0} \chi_{0}^{\prime}\right)^{1 / 2} \chi_{1} \chi_{2},
$$

and let $\tilde{B}_{r} \in \Psi_{\mathrm{b}}^{s+1}(X)$ with principal symbol $b_{r}$. Then let

$$
C \in \Psi_{\mathrm{b}}^{0}(X) \quad \text { and } \quad \sigma_{\mathrm{b}, 0}(C)=\left|\underline{\zeta}_{n-1}\right|^{-1} h^{1 / 2} \psi=\hat{h}^{1 / 2} \psi,
$$

where $\psi \in S_{\text {hom }}^{0}\left({ }^{\mathrm{b}} T^{*} X \backslash o\right.$ ) is identically 1 on $U$ considered as a subset of ${ }^{\mathrm{b}} S^{*} X$; recall from Remark 8.3 that $\hat{h}$ is bounded below by a positive quantity here. 
If $\tilde{C}_{r} \in \Psi_{\mathrm{b}}^{2 s}(X)$ with principal symbol

$$
\sigma_{\mathrm{b}, 2 s}\left(\tilde{C}_{r}\right)=-4 w_{r}^{2} \digamma^{-1} \delta^{-1} a\left|\underline{\zeta}_{n-1}\right|^{-1} \chi_{0}^{\prime} \chi_{1} \chi_{2}=-\left|\underline{\zeta}_{n-1}\right|^{-2} b_{r}^{2},
$$

then we deduce from $(8-13)-(8-20)$ that $^{6}$

$$
\begin{aligned}
& \qquad A_{r}^{*} A_{r} P-\imath P A_{r}^{*} A_{r} \\
& \quad=\tilde{B}_{r}^{*}\left(C^{*} x^{2} C+x R^{b} x+\left(x D_{x}\right)^{*} \tilde{R}^{\prime} x+x \tilde{R}^{\prime \prime}\left(x D_{x}\right)+\left(x D_{x}\right)^{*} R^{\sharp}\left(x D_{x}\right)\right) \tilde{B}_{r}+R_{r}^{\prime \prime}+E_{r}+E_{r}^{\prime}
\end{aligned}
$$

with

$$
\begin{gathered}
R^{\mathrm{b}} \in \Psi_{\mathrm{b}}^{0}(X), \quad \tilde{R}^{\prime}, \tilde{R}^{\prime \prime} \in \Psi_{\mathrm{b}}^{-1}(X), \quad R^{\sharp} \in \Psi_{\mathrm{b}}^{-2}(X), \\
R_{r}^{\prime \prime} \in L^{\infty}\left((0,1) ; \operatorname{Diff}_{0}^{2} \Psi_{\mathrm{b}}^{2 s-1}(X)\right), \quad E_{r}, E_{r}^{\prime} \in L^{\infty}\left((0,1) ; \operatorname{Diff}_{0}^{2} \Psi_{\mathrm{b}}^{2 s}(X)\right),
\end{gathered}
$$

with $\mathrm{WF}_{\mathrm{b}}^{\prime}(E) \subset \eta^{-1}((-\infty,-\delta]) \cap U$ and $\mathrm{WF}_{\mathrm{b}}^{\prime}\left(E^{\prime}\right) \cap \dot{\Sigma}=\varnothing$, and with $r^{\mathrm{b}}=\sigma_{\mathrm{b}, 0}\left(R^{\mathrm{b}}\right), \quad \tilde{r}^{\prime}=\sigma_{\mathrm{b},-1}\left(\tilde{R}^{\prime}\right)$, $\tilde{r}^{\prime \prime}=\sigma_{\mathrm{b},-1}\left(\tilde{R}^{\prime \prime}\right), r^{\sharp} \in \sigma_{\mathrm{b},-2}\left(R^{\sharp}\right)$, and

$$
\begin{array}{rlrl}
\left|r^{\mathrm{b}}\right| & \leq C_{2}\left(\delta \epsilon+\epsilon^{-1}+\delta \digamma^{-1}\right), & & \left|\underline{\zeta}_{n-1} \tilde{r}^{\prime}\right| \leq C_{2}\left(\delta \epsilon+\epsilon^{-1}+\delta \digamma^{-1}\right), \\
\left|\underline{\zeta}_{n-1} \tilde{r}^{\prime \prime}\right| & \leq C_{2}\left(\delta \epsilon+\epsilon^{-1}+\delta \digamma^{-1}\right), & \left|\underline{\zeta}_{n-1}^{2} r^{\sharp}\right| \leq C_{2}\left(\delta \epsilon+\epsilon^{-1}+\delta \digamma^{-1}\right) .
\end{array}
$$

This is almost completely analogous to [Vasy 2008c, Equation (6.18)] with the understanding that each term therein inside the parentheses attains an additional factor of $x^{2}$ (corresponding to $\square$ being in $\operatorname{Diff}_{0}^{2}(X)$ rather than $\left.\operatorname{Diff}^{2}(X)\right)$, which we partially include in $x D_{x}$ (vs. $D_{x}$ ). The only difference is the presence of the $\delta \digamma^{-1}$ term, which however is treated like the $\epsilon \delta$ term for $\digamma$ sufficiently large; hence the rest of the proof proceeds very similarly to that paper. We go through this argument to show the role that $\lambda$ and the Poincare inequality play, and in particular how the restrictions on $\lambda$ arise.

Having calculated the commutator, we proceed to estimate the "error terms" $R^{b}, \tilde{R}^{\prime}, \tilde{R}^{\prime \prime}$ and $R^{\sharp}$ as operators. We start with $R^{b}$. By the standard square root construction to prove the boundedness of PsDOs on $L^{2}$, see e.g. the discussion after [Vasy 2008c, Remark 2.1], there exists $R_{\mathrm{b}}^{\mathrm{b}} \in \Psi_{\mathrm{b}}^{-1}(X)$ such that

$$
\left\|R^{b} v\right\| \leq 2 \sup \left|r^{b}\right|\|v\|+\left\|R_{b}^{b} v\right\| \quad \text { for all } v \in L^{2}(X) .
$$

Here $\|\cdot\|$ is the $L^{2}(X)$ norm, as usual. Thus, we can estimate, for any $\gamma>0$,

$$
\begin{aligned}
\left|\left\langle R^{b} v, v\right\rangle\right| & \leq\left\|R^{b} v\right\|\|v\| \leq 2 \sup \left|r^{b}\right|\|v\|^{2}+\left\|R_{b}^{b} v\right\|\|v\| \\
& \leq 2 C_{2}\left(\delta \epsilon+\epsilon^{-1}+\delta \digamma^{-1}\right)\|v\|^{2}+\gamma^{-1}\left\|R_{b}^{b} v\right\|^{2}+\gamma\|v\|^{2} .
\end{aligned}
$$

Now we turn to $\tilde{R}^{\prime}$. Let $T \in \Psi_{\mathrm{b}}^{-1}(X)$ be elliptic (which we use to shift the orders of PsDOs at our convenience), with symbol $\left|\underline{\zeta}_{n-1}\right|^{-1}$ on $\operatorname{supp} a$, and with $T^{-} \in \Psi_{\mathrm{b}}^{1}(X)$ a parametrix, so $T^{-} T=\operatorname{Id}+F$

\footnotetext{
${ }^{6}$ The $f_{\sharp}^{\sharp}$ terms are included in $R^{\sharp}$, while the $f_{b}^{\sharp}$ terms are included in $E^{\prime}$, and similarly for the other analogous terms in $f^{\prime}, f^{\prime \prime}, f^{b}$. Moreover, in view of Lemma 5.4, we can freely rearrange factors, e.g., writing $C^{*} x^{2} C$ as $x C^{*} C x$ if we wish, with the exception of commuting powers of $x$ with $x D_{x}$ or $\left(x D_{x}\right)^{*}$ since we need to regard the latter as elements of $\operatorname{Diff}_{0}^{1}(X)$ rather than $\operatorname{Diff}_{\mathrm{b}}^{1}(X)$. Indeed, the difference between rearrangements has lower b-order than the product, in this case being in $x^{2} \Psi_{\mathrm{b}}^{-1}(X)$, which in view of Lemma 5.5, at the cost of dropping powers of $x$, can be translated into a gain in 0-order, that is, $x^{2} \Psi_{\mathrm{b}}^{-1}(X) \subset \operatorname{Diff}_{0}^{2} \Psi_{\mathrm{b}}^{-3}(X)$, with the result that these terms can be moved to the "error term" $R^{\prime \prime} \in L^{\infty}\left((0,1) ; \operatorname{Diff}_{0}^{2} \Psi_{\mathrm{b}}^{2 s-1}(X)\right)$.
} 
with $F \in \Psi_{\mathrm{b}}^{-\infty}(X)$. Then there exists $\tilde{R}_{\mathrm{b}}^{\prime} \in \Psi_{\mathrm{b}}^{-1}(X)$ such that

$$
\begin{aligned}
\left\|\left(\tilde{R}^{\prime}\right)^{*} w\right\| & =\left\|\left(\tilde{R}^{\prime}\right)^{*}\left(T^{-} T-F\right) w\right\| \leq\left\|\left(\left(\tilde{R}^{\prime}\right)^{*} T^{-}\right)(T w)\right\|+\left\|\left(\tilde{R}^{\prime}\right)^{*} F w\right\| \\
& \leq 2 C_{2}\left(\delta \epsilon+\epsilon^{-1}+\delta \digamma^{-1}\right)\|T w\|+\left\|\tilde{R}_{b}^{\prime} T w\right\|+\left\|\left(\tilde{R}^{\prime}\right)^{*} F w\right\|
\end{aligned}
$$

for all $w$ with $T w \in L^{2}(X)$, and similarly, there exists $\tilde{R}_{\mathrm{b}}^{\prime \prime} \in \Psi_{\mathrm{b}}^{-1}(X)$ such that

$$
\left\|\tilde{R}^{\prime \prime} w\right\| \leq 2 C_{2}\left(\delta \epsilon+\epsilon^{-1}+\delta \digamma^{-1}\right)\|T w\|+\left\|\tilde{R}_{b}^{\prime \prime} T w\right\|+\left\|\tilde{R}^{\prime \prime} F w\right\| .
$$

Finally, there exists $R_{\mathrm{b}}^{\sharp} \in \Psi_{\mathrm{b}}^{-1}(X)$ such that

$$
\left\|\left(T^{-}\right)^{*} R^{\sharp} w\right\| \leq 2 C_{2}\left(\delta \epsilon+\epsilon^{-1}+\delta \digamma^{-1}\right)\|T w\|+\left\|R_{b}^{\sharp} T w\right\|+\left\|\left(T^{-}\right)^{*} R^{\sharp} F w\right\|
$$

for all $w$ with $T w \in L^{2}(X)$. Thus,

$$
\begin{aligned}
\left|\left\langle x v,\left(\tilde{R}^{\prime}\right)^{*}\left(x D_{x}\right) v\right\rangle\right| \leq & 2 C_{2}\left(\delta \epsilon+\epsilon^{-1}+\delta \digamma^{-1}\right)\left\|T x D_{x} v\right\|\|x v\| \\
& +2 \gamma\|x v\|^{2}+\gamma^{-1}\left\|\tilde{R}_{b}^{\prime} T x D_{x} v\right\|^{2}+\gamma^{-1}\left\|F^{\prime} x D_{x} v\right\|^{2}, \\
\left|\left\langle\tilde{R}^{\prime \prime} x D_{x} v, x v\right\rangle\right| \leq 2 & C_{2}\left(\delta \epsilon+\epsilon^{-1}+\delta \digamma^{-1}\right)\left\|T x D_{x} v\right\|\|x v\| \\
+ & 2 \gamma\|x v\|^{2}+\gamma^{-1}\left\|\tilde{R}_{b}^{\prime \prime} T x D_{x} v\right\|^{2}+\gamma^{-1}\left\|F^{\prime \prime} x D_{x} v\right\|^{2},
\end{aligned}
$$

and, writing $x D_{x} v=T^{-} T\left(x D_{x} v\right)-F\left(x D_{x} v\right)$ in the right factor, and taking the adjoint of $T^{-}$,

$$
\begin{aligned}
\left|\left\langle R^{\sharp} x D_{x} v, x D_{x} v\right\rangle\right| \leq 2 C_{2}(\delta \epsilon+ & \left.\epsilon^{-1}+\delta \digamma^{-1}\right)\left\|T\left(x D_{x}\right) v\right\|\left\|T\left(x D_{x}\right) v\right\|+2 \gamma\left\|T\left(x D_{x}\right) v\right\|^{2} \\
& +\gamma^{-1}\left\|R_{b}^{\sharp} T\left(x D_{x}\right) v\right\|^{2}+\gamma^{-1}\left\|F\left(x D_{x}\right) v\right\|^{2}+\left\|R^{\sharp}\left(x D_{x}\right) v\right\|\left\|F^{\sharp}\left(x D_{x} v\right)\right\|,
\end{aligned}
$$

with $F^{\prime}, F^{\prime \prime}, F^{\sharp} \in \Psi_{\mathrm{b}}^{-\infty}(X)$.

Now, by (8-21),

$$
\begin{aligned}
\left\langle l\left[A_{r}^{*} A_{r}, P\right] u, u\right\rangle=\left\|C x \tilde{B}_{r} u\right\|^{2} & +\left\langle R^{\mathrm{b}} x \tilde{B}_{r} u, x \tilde{B}_{r} u\right\rangle+\left\langle\tilde{R}^{\prime \prime} x D_{x} \tilde{B}_{r} u, x \tilde{B}_{r} u\right\rangle \\
+\left\langle x \tilde{B}_{r} u,\left(\tilde{R}^{\prime}\right)^{*} x D_{x} \tilde{B}_{r} u\right\rangle+\left\langle R^{\sharp} x D_{x} \tilde{B}_{r} u, x D_{x} \tilde{B}_{r} u\right\rangle & +\left\langle R_{r}^{\prime \prime} u, u\right\rangle+\left\langle\left(E_{r}+E_{r}^{\prime}\right) u, u\right\rangle
\end{aligned}
$$

On the other hand, this commutator can be expressed as in (8-12), so

$$
\begin{aligned}
& \left\langle\imath A_{r}^{*} A_{r} P u, u\right\rangle-\left\langle l A_{r}^{*} A_{r} u, P u\right\rangle \\
& =-2 \operatorname{Im} \lambda\left\|A_{r} u\right\|^{2}+\left\|C x \tilde{B}_{r} u\right\|^{2}+\left\langle R^{b} x \tilde{B}_{r} u, x \tilde{B}_{r} u\right\rangle+\left\langle\tilde{R}^{\prime \prime} x D_{x} \tilde{B}_{r} u, x \tilde{B}_{r} u\right\rangle \\
& \quad+\left\langle x \tilde{B}_{r} u,\left(\tilde{R}^{\prime}\right)^{*} x D_{x} \tilde{B}_{r} u\right\rangle+\left\langle R^{\sharp} x D_{x} \tilde{B}_{r} u, x D_{x} \tilde{B}_{r} u\right\rangle+\left\langle R_{r}^{\prime \prime} u, u\right\rangle+\left\langle\left(E_{r}+E_{r}^{\prime}\right) u, u\right\rangle,
\end{aligned}
$$

so the signs of the first two terms agree if $\operatorname{Im} \lambda<0$, and the $\operatorname{Im} \lambda$ term vanishes if $\lambda$ is real.

Assume for the moment that $\mathrm{WF}_{\mathrm{b}}^{-1, s+3 / 2}(P u) \cap U=\varnothing$ - this is certainly the case in our setup if $q_{0} \notin \mathrm{WF}_{\mathrm{b}}^{-1, \infty}(P u)$, but this assumption is a little stronger than $q_{0} \notin \mathrm{WF}_{\mathrm{b}}^{-1, s+1}(P u)$, which is what we need to assume for the second paragraph in the statement of the proposition. We deal with the weakened hypothesis $q_{0} \notin \mathrm{WF}_{\mathrm{b}}^{-1, s+1}(P u)$ at the end of the proof. Returning to (8-23), the utility of the commutator 
calculation is that we have good information about $P u$ (this is where we use that we have a microlocal solution of the PDE!). Namely, we estimate the left hand side as

$$
\begin{aligned}
\left|\left\langle A_{r} P u, A_{r} u\right\rangle\right| & \leq\left|\left\langle\left(T^{-}\right)^{*} A_{r} P u, T A_{r} u\right\rangle\right|+\left|\left\langle A_{r} P u, F A_{r} u\right\rangle\right| \\
& \leq\left\|\left(T^{-}\right)^{*} A_{r} P u\right\|_{H_{0}^{-1}(X)}\left\|T A_{r} u\right\|_{H_{0}^{1}(X)}+\left\|A_{r} P u\right\|_{H_{0}^{-1}(X)}\left\|F A_{r} u\right\|_{H_{0}^{1}(X)} .
\end{aligned}
$$

Since $\left(T^{-}\right)^{*} A_{r}$ is uniformly bounded in $\Psi_{\mathrm{bc}}^{s+3 / 2}(X)$ and $T A_{r}$ is uniformly bounded in $\Psi_{\mathrm{bc}}^{s-1 / 2}(X)$, both with $\mathrm{WF}_{\mathrm{b}}^{\prime}$ in $U$, with $\mathrm{WF}_{\mathrm{b}}^{-1, s+3 / 2}(P u)$ and $\mathrm{WF}_{\mathrm{b}}^{1, s-1 / 2}(u)$, respectively, disjoint from them, we deduce (using Lemma 5.14 and its $H_{0}^{-1}$ analogue) that $\left|\left\langle\left(T^{-}\right)^{*} A_{r} P u, T A_{r} u\right\rangle\right|$ is uniformly bounded. Similarly, taking into account that $F A_{r}$ is uniformly bounded in $\Psi_{\mathrm{b}}^{-\infty}(X)$, we see that $\left|\left\langle A_{r} P u, F A_{r} u\right\rangle\right|$ is also uniformly bounded, so $\left|\left\langle A_{r} P u, A_{r} u\right\rangle\right|$ is uniformly bounded for $r \in(0,1]$.

Thus,

$$
\begin{gathered}
\left\|C x \tilde{B}_{r} u\right\|^{2}-\operatorname{Im} \lambda\left\|A_{r} u\right\|^{2} \\
\leq 2\left|\left\langle A_{r} P u, A_{r} u\right\rangle\right|+\left|\left\langle\left(E_{r}+E_{r}^{\prime}\right) u, u\right\rangle\right|+\left(2 C_{2}\left(\delta \epsilon+\epsilon^{-1}+\delta \digamma^{-1}\right)+\gamma\right)\left\|x \tilde{B}_{r} u\right\|^{2}+\gamma^{-1}\left\|R_{b}^{b} x \tilde{B}_{r} u\right\|^{2} \\
+4 C_{2}\left(\delta \epsilon+\epsilon^{-1}+\delta \digamma^{-1}\right)\left\|x \tilde{B}_{r} u\right\|\left\|T\left(x D_{x}\right) \tilde{B}_{r} u\right\|+\gamma^{-1}\left\|\tilde{R}_{b}^{\prime} T\left(x D_{x}\right) \tilde{B}_{r} u\right\|^{2}+\gamma^{-1}\left\|\tilde{R}_{b}^{\prime \prime} T\left(x D_{x}\right) \tilde{B}_{r} u\right\|^{2} \\
+4 \gamma\left\|x \tilde{B}_{r} u\right\|^{2}+\left(2 C_{2}\left(\delta \epsilon+\epsilon^{-1}+\delta \digamma^{-1}\right)+2 \gamma\right)\left\|T\left(x D_{x}\right) \tilde{B}_{r} u\right\|^{2} \\
+\gamma^{-1}\left\|R_{b}^{\sharp} T\left(x D_{x}\right) \tilde{B}_{r} u\right\|^{2}+\left\|R^{\sharp}\left(x D_{x}\right) \tilde{B}_{r} u\right\|\left\|F\left(x D_{x}\right) \tilde{B}_{r} u\right\| \\
\quad+\gamma^{-1}\left\|F\left(x D_{x}\right) \tilde{B}_{r} u\right\|^{2}+\gamma^{-1}\left\|F^{\prime}\left(x D_{x}\right) \tilde{B}_{r} u\right\|^{2}+\gamma^{-1}\left\|F^{\prime \prime}\left(x D_{x}\right) \tilde{B}_{r} u\right\|^{2} .
\end{gathered}
$$

All terms but the ones involving $C_{2}$ or $\gamma\left(\right.$ not $\left.\gamma^{-1}\right)$ remain bounded as $r \rightarrow 0$. The $C_{2}$ and $\gamma$ terms can be estimated by writing $T\left(x D_{x}\right)=\left(x D_{x}\right) T^{\prime}+T^{\prime \prime}$ for some $T^{\prime}, T^{\prime \prime} \in \Psi_{\mathrm{b}}^{-1}(X)$, and using Lemma 7.3 and the Poincaré lemma where necessary. Namely, we use either $\operatorname{Im} \lambda \neq 0$ or $\lambda<(n-1)^{2} / 4$ to control $x D_{x} L \tilde{B}_{r} u$ and $L \tilde{B}_{r} u$ in $L^{2}(X)$ in terms of $\left\|x \tilde{B}_{r} u\right\|_{L^{2}}$ where $L \in \Psi_{\mathrm{b}}^{-1}(X)$; this is possible by factoring $D_{y_{n-1}}$ (which is elliptic on $\left.\mathrm{WF}^{\prime}\left(\tilde{B}_{r}\right)\right)$ out of $\tilde{B}_{r}$ modulo an error $\tilde{F}_{r}$ bounded in $\Psi_{\mathrm{bc}}^{s}(X)$, which in turn can be incorporated into the "error" given by the right hand side of Lemma 7.3. Thus, there exists $C_{3}>0, G \in \Psi_{\mathrm{b}}^{s-1 / 2}(X)$ and $\tilde{G} \in \Psi_{\mathrm{b}}^{s+1 / 2}(X)$ as in Lemma 7.3 such that

$\left\|x D_{x} L \tilde{B}_{r} u\right\|^{2}+\left\|L \tilde{B}_{r} u\right\| \leq C_{3}\left(\left\|x \tilde{B}_{r} u\right\|^{2}+\|u\|_{H_{0, \mathrm{~b}, \mathrm{loc}}^{1, k}(X)}^{2}+\|G u\|_{H_{0}^{1}(X)}^{2}+\|P u\|_{H_{0, \mathrm{~b}, \mathrm{loc}}^{-1, k}(X)}^{2}+\|\tilde{G} P u\|_{H_{0}^{-1}(X)}^{2}\right)$.

We further estimate $\left\|x \tilde{B}_{r} u\right\|$ in terms of $\left\|C x \tilde{B}_{r} u\right\|$ and $\|u\|_{H_{0, \text { loc }}^{1}(X)}$ using that $C$ is elliptic on $\mathrm{WF}_{\mathrm{b}}^{\prime}(B)$ and Lemma 5.14. We conclude, using $\operatorname{Im} \lambda \leq 0$, taking $\epsilon$ sufficiently large, then $\gamma$ and $\delta_{0}$ sufficiently small, and finally $\digamma$ sufficiently large, that there exist $\gamma>0, \epsilon>0, \delta_{0}>0$ and $C_{4}>0$ and $C_{5}>0$ such that for $\delta \in\left(0, \delta_{0}\right)$,

$$
\begin{aligned}
C_{4}\left\|x \tilde{B}_{r} u\right\|^{2} \leq 2\left|\left\langle A_{r} P u, A_{r} u\right\rangle\right| & +\left|\left\langle\left(E_{r}+E_{r}^{\prime}\right) u, u\right\rangle\right| \\
& +C_{5}\left(\|G u\|_{H_{0}^{1}(X)}^{2}+\|\tilde{G} P u\|_{H_{0}^{-1}(X)}^{2}\right)+C_{5}\left(\|u\|_{H_{0, b, \mathrm{loc}}^{1, k}(X)}+\|P u\|_{H_{0, \mathrm{~b}, \mathrm{loc}}^{-1, k}(X)}\right) .
\end{aligned}
$$

Letting $r \rightarrow 0$ now keeps the right hand side bounded, proving that $\left\|x \tilde{B}_{r} u\right\|$ is uniformly bounded as $r \rightarrow 0$; hence $x \tilde{B}_{0} u \in L^{2}(X)$ (see the proof of Proposition 7.7). In view of Lemma 7.3 and the Poincaré 
inequality (as in the proof of Proposition 7.7), this proves that $q_{0} \notin \mathrm{WF}_{\mathrm{b}}^{1, s}(u)$, and hence proves the first statement of the proposition.

In fact, recalling that we needed $q_{0} \notin \mathrm{WF}_{\mathrm{b}}^{-1, s+3 / 2}(P u)$ for the uniform boundedness in (8-24), this proves a slightly weaker version of the second statement of the proposition with $\mathrm{WF}_{\mathrm{b}}^{-1, s+1}(P u)$ replaced by $\mathrm{WF}_{\mathrm{b}}^{-1, s+3 / 2}(\mathrm{Pu})$. For the more precise statement we modify (8-24) - this is the only term in $(8-25)$ that needs modification to prove the optimal statement. Let $\tilde{T} \in \Psi_{\mathrm{b}}^{-1 / 2}(X)$ be elliptic, $\tilde{T}^{-} \in \Psi_{\mathrm{b}}^{1 / 2}(X)$ a parametrix, with $\tilde{F}=\tilde{T}^{-} \tilde{T}-\operatorname{Id} \in \Psi_{\mathrm{b}}^{-\infty}(X)$. Then, similarly to (8-24), we have for any $\gamma>0$,

$$
\begin{aligned}
\left|\left\langle A_{r} P u, A_{r} u\right\rangle\right| & \leq\left|\left\langle\left(\tilde{T}^{-}\right)^{*} A_{r} P u, \tilde{T} A_{r} u\right\rangle\right|+\left|\left\langle A_{r} P u, \tilde{F} A_{r} u\right\rangle\right| \\
& \leq \gamma^{-1}\left\|\left(\tilde{T}^{-}\right)^{*} A_{r} P u\right\|_{H_{0}^{-1}(X)}^{2}+\gamma\left\|\tilde{T} A_{r} u\right\|_{H^{1}(X)}^{2}+\left\|A_{r} P u\right\|_{H^{-1}(X)}\left\|\tilde{F} A_{r} u\right\|_{H_{0}^{1}(X)} .
\end{aligned}
$$

The last term on the right hand side can be estimated as before. As $\left(\tilde{T}^{-}\right)^{*} A_{r}$ is bounded in $\Psi_{\mathrm{bc}}^{s+1}(X)$ with $\mathrm{WF}_{\mathrm{b}}^{\prime}$ disjoint from $U$, we see that $\left\|\left(\tilde{T}^{-}\right)^{*} A_{r} P u\right\|_{H_{0}^{-1}(X)}$ is uniformly bounded. Moreover, $\left\|\tilde{T} A \Lambda_{r} u\right\|_{H_{0}^{1}(X)}^{2}$ can be estimated, using Lemma 7.3 and the Poincaré inequality, by $\left\|x D_{y_{n-1}} \tilde{T} A \Lambda_{r} u\right\|_{L^{2}(X)}^{2}$ modulo terms that are uniformly bounded as $r \rightarrow 0$. The principal symbol of $D_{y_{n-1}} \tilde{T} A$ is $\zeta_{n-1} \sigma_{\mathrm{b},-1 / 2}(\tilde{T}) a$, with $a=\chi_{0} \chi_{1} \chi_{2}$, where $\chi_{0}$ stands for $\chi_{0}\left(A_{0}^{-1}(2-\phi / \delta)\right)$, etc., so we can write

$$
\left|\underline{\zeta}_{n-1}\right|^{1 / 2} a=\left|\underline{\zeta}_{n-1}\right|^{1 / 2} \chi_{0} \chi_{1} \chi_{2}=A_{0}^{-1}(2-\phi / \delta)\left|\underline{\zeta}_{n-1}\right|^{1 / 2}\left(\chi_{0} \chi_{0}^{\prime}\right)^{1 / 2} \chi_{1} \chi_{2}=\digamma^{-1 / 2} \delta^{1 / 2}(2-\phi / \delta) \tilde{b},
$$

where we used that

$$
\chi_{0}^{\prime}\left(\digamma^{-1}(2-\phi / \delta)\right)=\digamma^{2}(2-\phi / \delta)^{-2} \chi_{0}\left(\digamma^{-1}(2-\phi / \delta)\right)
$$

when $2-\phi / \delta>0$, while $a$ and $\tilde{b}$ vanish otherwise. Correspondingly, using that $\left|\zeta_{n-1}\right|^{1 / 2} \sigma_{\mathrm{b},-1 / 2}(\tilde{T})$ is $\mathscr{C}^{\infty}$ and homogeneous degree zero near the support of $a$ in ${ }^{\mathrm{b}} T^{*} X \backslash o$, we can write $D_{y_{n-1}} \tilde{T} A=G \tilde{B}+F$, with $G \in \Psi_{\mathrm{b}}^{0}(X)$ and $F \in \Psi_{\mathrm{b}}^{-1 / 2}(X)$. Thus, modulo terms that are bounded as $r \rightarrow 0$, we can estimate $\left\|x D_{y_{n-1}} \tilde{T} A \Lambda_{r} u\right\|^{2}$ (and hence $\left\|\tilde{T} A \Lambda_{r} u\right\|_{H_{0}^{1}(X)}^{2}$ ) from above by $C_{6}\left\|x \tilde{B}_{r} u\right\|^{2}$. Therefore, modulo terms that are bounded as $r \rightarrow 0$, for $\gamma>0$ sufficiently small, $\gamma\left\|\tilde{T} A_{r} u\right\|_{H_{0}^{1}(X)}^{2}$ can be absorbed into $\left\|C x \tilde{B}_{r} u\right\|^{2}$. As the treatment of the other terms on the right hand side of (8-25) requires no change, we deduce as above that $x \tilde{B}_{0} u \in L^{2}(X)$, which (in view of Lemma 7.3) proves that $q_{0} \notin \mathrm{WF}_{\mathrm{b}}^{1, s}(u)$, completing the proof of the iterative step.

We need to make one more remark to prove the proposition for $\mathrm{WF}_{\mathrm{b}}^{1, \infty}(u)$; namely we need to show that the neighborhoods of $q_{0}$ that are disjoint from $\mathrm{WF}_{\mathrm{b}}^{1, s}(u)$ do not shrink uncontrollably to $\left\{q_{0}\right\}$ as $s \rightarrow \infty$. This argument parallels the last paragraph of the proof of [Hörmander 1985, Proposition 24.5.1]. In fact, note that above we have proved that the elliptic set of $\tilde{B}=\tilde{B}_{s}$ is disjoint from $\mathrm{WF}_{\mathrm{b}}^{1, s}(u)$. In the next step, when we are proving $q_{0} \notin \mathrm{WF}_{\mathrm{b}}^{1, s+1 / 2}(u)$, we decrease $\delta>0$ slightly (by an arbitrary small amount), thus decreasing the support of $a=a_{s+1 / 2}$ in (8-8), to make sure that $\operatorname{supp} a_{s+1 / 2}$ is a subset of the elliptic set of the union of $\tilde{B}_{s}$ with the region $\eta<0$, and hence that $\mathrm{WF}_{\mathrm{b}}^{1, s}(u) \cap \operatorname{supp} a_{s+1 / 2}=\varnothing$. Each iterative step thus shrinks the elliptic set of $\tilde{B}_{s}$ by an arbitrarily small amount, which allows us to conclude that $q_{0}$ has a neighborhood $U^{\prime}$ such that $\mathrm{WF}_{\mathrm{b}}^{1, s}(u) \cap U^{\prime}=\varnothing$ for all $s$. This proves that 
$q_{0} \notin \mathrm{WF}_{\mathrm{b}}^{1, \infty}(u)$, and indeed that $\mathrm{WF}_{\mathrm{b}}^{1, \infty}(u) \cap U^{\prime}=\varnothing$, for if $A \in \Psi_{\mathrm{b}}^{m}(X)$ with $\mathrm{WF}_{\mathrm{b}}^{\prime}(A) \subset U^{\prime}$, then $A u \in H_{0}^{1}(X)$ by Lemma 5.10 and Corollary 5.12.

Before turning to tangential propagation we need a technical lemma, which roughly states that when applied to solutions of $P u=0$ with $u \in H_{0}^{1}(X)$, the operators $x D_{x}$ and Id are not merely bounded by $x D_{y_{n-1}}$ microlocally, but are small compared to it, provided that $\lambda \in \mathbb{C} \backslash\left[(n-1)^{2} / 4, \infty\right)$. This result is the analogue of [Vasy 2008c, Lemma 7.1], and is proved as there, with the only difference being that the term $\left\langle\lambda A_{r} u, A_{r} u\right\rangle$ cannot be dropped; instead it is treated just as in Proposition 7.7 above. Below a $\delta$-neighborhood refers to a $\delta$-neighborhood with respect to the metric associated to any Riemannian metric on the manifold ${ }^{\mathrm{b}} T^{*} X$, and we identify ${ }^{\mathrm{b}} S^{*} X$ as the unit ball bundle with respect to some fiber metric on ${ }^{\mathrm{b}} T^{*} X$.

Lemma 8.4 (see [Vasy 2008c, Lemma 7.1]). Suppose that $P=\square_{g}+\lambda$, with $\lambda \in \mathbb{C} \backslash\left[(n-1)^{2} / 4, \infty\right)$. Suppose $u \in H_{0, b, l o c}^{1, k}(X)$, and suppose that we are given $K \subset{ }^{b} S^{*} X$ compact satisfying

$$
K \subset \mathscr{G} \cap T^{*} Y \backslash \mathrm{WF}_{\mathrm{b}}^{-1, s+1 / 2}(P u) .
$$

Then there exist $\delta_{0}>0$ and $C_{0}>0$ with the following property. Let $\delta<\delta_{0}$. Let $U \subset{ }^{\mathrm{b}}{ }^{*} X$ be open in a $\delta$-neighborhood of $K$, and let $\mathscr{A}=\left\{A_{r}: r \in(0,1]\right\}$ be a bounded family of PsDOs in $\Psi_{\mathrm{bc}}^{s}(X)$ with $\mathrm{WF}_{\mathrm{b}}^{\prime}(\mathscr{A}) \subset U$, and with $A_{r} \in \Psi_{\mathrm{b}}^{s-1}(X)$ for $r \in(0,1]$.

Then there exist $G \in \Psi_{\mathrm{b}}^{s-1 / 2}(X)$ and $\tilde{G} \in \Psi_{\mathrm{b}}^{s+1 / 2}(X)$ with $\mathrm{WF}_{\mathrm{b}}^{\prime}(G), \mathrm{WF}_{\mathrm{b}}^{\prime}(\tilde{G}) \subset U$ and $\tilde{C}_{0}=\tilde{C}_{0}(\delta)>0$ such that for all $r>0$,

$$
\begin{aligned}
& \left\|x D_{x} A_{r} u\right\|^{2}+\left\|A_{r} u\right\|^{2} \\
& \quad \leq C_{0} \delta\left\|x D_{y_{n-1}} A_{r} u\right\|^{2}+\tilde{C}_{0}\left(\|u\|_{H_{0, b, l o c}^{1, k}(X)}^{2}+\|G u\|_{H_{0}^{1}(X)}^{2}+\|P u\|_{H_{0, b, l o c}^{-1, k}(X)}^{2}+\|\tilde{G} P u\|_{H_{0}^{-1}(X)}^{2}\right) .
\end{aligned}
$$

The meaning of $\|u\|_{H_{0, \mathrm{~b}, \mathrm{loc}}^{1, k}(X)}$ and $\|P u\|_{H_{0, \mathrm{~b}, \mathrm{loc}}^{-1, k}(X)}^{2}$ is stated in Remark 7.2.

Remark 8.5. Since $K$ is compact, this is essentially a local result. In particular, we may assume that $K$ is a subset of ${ }^{\mathrm{b}} T^{*} X$ over a suitable local coordinate patch. Moreover, we may assume that $\delta_{0}>0$ is sufficiently small so that $D_{y_{n-1}}$ is elliptic on $U$.

Proof. By Lemma 7.3 applied with $K$ replaced by $\mathrm{WF}_{\mathrm{b}}^{\prime}(\mathscr{A})$ in the hypothesis (note that the latter is compact), we already know that

$$
\left|\left\langle d A_{r} u, d A_{r} u\right\rangle_{G}+\lambda\left\|A_{r} u\right\|^{2}\right| \leq C_{0}^{\prime}\left(\|u\|_{H_{0, b, \mathrm{loc}}^{1, k}(X)}^{2}+\|G u\|_{H_{0}^{1}(X)}^{2}+\|P u\|_{H_{0, b, \mathrm{loc}}^{-1, k)}}^{2}+\|\tilde{G} P u\|_{H_{0}^{-1}(X)}^{2}\right) .
$$

for some $C_{0}^{\prime}>0$ and for some $G$ and $\tilde{G}$ as in the statement of the lemma. Freezing the coefficients at $Y$, as in the proof of Proposition 7.7 — see [Vasy 2008c, Lemma 7.1] for details — we deduce that

$$
\begin{aligned}
& \mid\left\|x D_{x} A_{r} u\right\|^{2}- \lambda\left\|A_{r} u\right\|^{2} \mid \\
& \leq \int_{X}\left(B_{i j}(0, y)\left(x D_{y_{i}}\right) A_{r} u \overline{\left(x D_{y_{j}}\right) A_{r} u}\right)|d g|+C_{1} \delta\left\|x D_{y_{n-1}} A_{r} u\right\|^{2} \\
& \quad+C_{0}^{\prime \prime}\left(\|u\|_{H_{0, b, l o c}^{1, k}(X)}^{2}+\|G u\|_{H_{0}^{1}(X)}^{2}+\|P u\|_{H_{0, b, \mathrm{loc}}^{-1, k}(X)}^{2}+\|\tilde{G} P u\|_{H^{-1}(X)}^{2}\right) .
\end{aligned}
$$


Now, one can show that

$$
\begin{aligned}
\left|\int_{X}\left(\sum\left(D_{y_{i}}^{*} B_{i j}(0, y) D_{y_{j}}\right) x A_{r} u \overline{x A_{r} u}\right)\right| d g|| \\
\leq C_{2} \delta\left\|D_{y_{n-1}} A_{r} u\right\|^{2}+\tilde{C}_{2}(\delta)\left(\|u\|_{H_{0, \mathrm{~b}, \mathrm{loc}}^{1, k}(X)}^{2}+\|G u\|_{H_{0}^{1}(X)}^{2}\right)
\end{aligned}
$$

precisely as in the proof of [Vasy 2008c, Lemma 7.1]. Equations (8-29)-(8-30) imply (8-27) with the left side replaced by $\left|\left\|x D_{x} A_{r} u\right\|^{2}-\lambda\left\|A_{r} u\right\|^{2}\right|$. If $\operatorname{Im} \lambda \neq 0$, we get the desired bound for $\left\|A_{r} u\right\|^{2}$ by taking the imaginary part of $\left\|x D_{x} A_{r} u\right\|^{2}-\lambda\left\|A_{r} u\right\|^{2}$; hence taking the real part gives the desired bound for $\left\|x D_{x} A_{r} u\right\|^{2}$ as well. If $\operatorname{Im} \lambda=0$ but $\lambda<(n-1)^{2} / 4$, we finish the proof using the Poincaré inequality; see the proof of Proposition 7.7.

We finally state the tangential, or glancing, propagation result.

Proposition 8.6 (tangential, or glancing, propagation). Suppose $P=\square_{g}+\lambda$ with $\lambda \in \mathbb{C} \backslash\left[(n-1)^{2} / 4, \infty\right)$. Let $U_{0}$ be a coordinate chart in $X$, and let $U$ be open with $\bar{U} \subset U_{0}$. Let $u \in H_{0, \mathrm{~b}, \mathrm{loc}}^{1, k}(X)$ for some $k \leq 0$, and let $\tilde{\pi}: T^{*} X \rightarrow T^{*} Y$ be the coordinate projection

$$
\tilde{\pi}:(x, y, \xi, \zeta) \mapsto(y, \zeta)
$$

Given $K \subset{ }^{\mathrm{b}} S_{U}^{*} X$ compact with

$$
K \subset\left(G \cap{ }^{\mathrm{b}} T_{Y}^{*} X\right) \backslash \mathrm{WF}_{\mathrm{b}}^{-1, \infty}(f), \quad \text { where } f=P u,
$$

there exist constants $C_{0}>0$ and $\delta_{0}>0$ such that the following holds. If $\operatorname{Im} \lambda \leq 0, q_{0}=\left(y_{0}, \underline{\zeta}_{0}\right) \in K$, $\alpha_{0}=\hat{\pi}^{-1}\left(q_{0}\right)$ and $W_{0}=\left.\tilde{\pi}_{*}\right|_{\alpha_{0}} \mathrm{H}_{p}$ considered as a constant vector field in local coordinates, and for some $0<\delta<\delta_{0}, C_{0} \delta \leq \epsilon<1$ and for all $\alpha=(x, y, \xi, \zeta) \in \Sigma$, there holds

$$
\pi(\alpha) \notin \mathrm{WF}_{\mathrm{b}}^{1, \infty}(u) \quad \text { if } \quad \alpha \in T^{*} X \text { and }\left|\tilde{\pi}\left(\alpha-\left(\alpha_{0}-\delta W_{0}\right)\right)\right| \leq \epsilon \delta \text { and }|x(\alpha)| \leq \epsilon \delta,
$$

then $q_{0} \notin \mathrm{WF}_{\mathrm{b}}^{1, \infty}(u)$.

In addition, $\mathrm{WF}_{\mathrm{b}}^{-1, \infty}(f)$ may be replaced by $\mathrm{WF}_{\mathrm{b}}^{-1, s+1}(f)$, and $\mathrm{WF}_{\mathrm{b}}^{1, \infty}(u)$ may be replaced by $\mathrm{WF}_{\mathrm{b}}^{1, s}(u), s \in \mathbb{R}$.

Remark 8.7. Just like Proposition 8.2, this result gives regularity propagation in the forward direction along $W_{0}$, that is, to conclude regularity at $q_{0}$, one needs to know regularity in the backward $W_{0}$-direction from $q_{0}$.

One can again change the direction of propagation, that is, replace $\delta$ by $-\delta$ in $\alpha-\left(\alpha_{0}-\delta W_{0}\right)$, provided one also changes the sign of $\operatorname{Im} \lambda$ to $\operatorname{Im} \lambda \geq 0$. In particular, if $\operatorname{Im} \lambda=0$, one obtains propagation estimates in both the forward and backward directions.

Proof. The proof follows closely that of [Vasy 2008c, Proposition 7.3], which is corrected at a point in [Vasy 2008a], so we merely point out the main steps. Again, one uses a commutant $A \in \Psi_{\mathrm{b}}^{0}(X)$ and weights $\Lambda_{r} \in \Psi_{\mathrm{b}}^{0}(X)$ for $r \in(0,1)$, uniformly bounded in $\Psi_{\mathrm{bc}}^{s+1 / 2}(X)$, with $A_{r}=A \Lambda_{r}$, in order to obtain the propagation of $\mathrm{WF}_{\mathrm{b}}^{1, s}(u)$ with the notation of that paper, whose analogue is $\mathrm{WF}_{\mathrm{b}}^{1, s}(u)$ here (the difference is the space relative to which one obtains b-regularity: it is $H^{1}(X)$ in the previous paper, but 
the zero-Sobolev space $H_{0}^{1}(X)$ here). One can use exactly the same commutants as in [Vasy 2008c], with a small correction given in [Vasy 2008a]. Then Proposition 8.1 lets one calculate $\iota\left[A_{r}^{*} A_{r}, P\right]$ to obtain a completely analogous expression to the formulas below [Vasy 2008c, Equation (7.16)], as corrected. The rest of the argument is completely analogous as well. Again, we refer the reader to [Vasy 2010a] because the commutator calculation is written up exactly as above in Proposition 8.1 (see [Vasy 2010a, Proposition 3.10]) and it is used subsequently in 6.1 there the same way it needs to be used here - any modifications are analogous to those in Proposition 8.2 and arise due to the nonnegligible nature of $\lambda$.

Again, we first construct the symbol $a$ of our commutator following the (corrected) proof [Vasy 2008c, Proposition 7.3]. Note that (with $\tilde{p}=x^{-2} \sigma_{\mathrm{b}, 2}(\tilde{P})=h$ )

$$
W_{0}\left(q_{0}\right)=\mathrm{H}_{\tilde{p}}\left(q_{0}\right) \text {, }
$$

and let

$$
W=\left|\underline{\zeta}_{n-1}\right|^{-1} W_{0},
$$

so $W$ is homogeneous of degree zero (with respect to the $\mathbb{R}^{+}$-action on the fibers of $T^{*} Y \backslash o$ ). We use

$$
\tilde{\eta}=\left(\operatorname{sgn}\left(\underline{\zeta}_{n-1}\right)_{0}\right)\left(y_{n-1}-\left(y_{n-1}\right)_{0}\right)
$$

now to measure propagation, since $\underline{\zeta}_{n-1}^{-1} \mathrm{H}_{\tilde{p}}\left(y_{n-1}\right)=2>0$ at $q_{0}$ by (6-7), so $\mathrm{H}_{\tilde{p}} \tilde{\eta}$ is $2\left|\underline{\zeta}_{n-1}\right|>0$ at $q_{0}$. Note that $\tilde{\eta}$ is thus increasing along GBB of $\hat{g}$.

First, we require

$$
\rho_{1}=\tilde{p}(y, \underline{\hat{\zeta}})=\left|\underline{\zeta_{n-1}}\right|^{-2} \tilde{p}(y, \underline{\zeta}) ;
$$

note that $d \rho_{1} \neq 0$ at $q_{0}$ for $\underline{\zeta} \neq 0$ there, but $\mathrm{H}_{\tilde{p}} \tilde{p} \equiv 0$, so

$$
W \rho_{1}\left(q_{0}\right)=0 .
$$

Next, $\operatorname{dim} T^{*} Y=2 n-2$ since $\operatorname{dim} Y=n-1$; hence $\operatorname{dim} S^{*} Y=2 n-3$. With a slight abuse of notation, we also regard $q_{0}$ as a point in $S^{*} Y$ - recall that $S^{*} Y=\left(T^{*} Y \backslash o\right) / \mathbb{R}^{+}$. We can also regard $W$ as a vector field on $S^{*} Y$ in view of its homogeneity. Since $W$ does not vanish as a vector in $T_{q_{0}} S^{*} Y$ in view of $W \tilde{\eta}\left(q_{0}\right) \neq 0$ since $\tilde{\eta}$ is homogeneous degree zero and hence a function on $S^{*} Y$, the kernel of $W$ in $T_{q_{0}}^{*} S^{*} Y$ has dimension $2 n-4$. Thus there exist homogeneous degree zero functions $\rho_{j}$ for $j=2, \ldots, 2 n-4$ on $T^{*} Y$ (and hence functions on $S^{*} Y$ ) such that

$$
\begin{aligned}
\rho_{j}\left(q_{0}\right)=0 & \text { for } j=2, \ldots, 2 n-4, \\
W \rho_{j}\left(q_{0}\right)=0 & \text { for } j=2, \ldots, 2 n-4, \\
d \rho_{j}\left(q_{0}\right) & \text { for } j=1, \ldots, 2 n-4 \text { are linearly independent at } q_{0} .
\end{aligned}
$$

By dimensional considerations, the $d \rho_{j}\left(q_{0}\right)$ for $j=1, \ldots, 2 n-4$, together with $d \tilde{\eta}$, span the cotangent space of $S^{*} Y$ at $q_{0}$, that is, of the quotient of $T^{*} Y$ by the $\mathbb{R}^{+}$-action, so the $\rho_{j}$, together with $\tilde{\eta}$, can be used as local coordinates on a chart $\tilde{U}_{0} \subset S^{*} Y$ near $q_{0}$. We also let $\tilde{u}$ be a neighborhood of $q_{0}{ }^{\text {in }}{ }^{\mathrm{b}} S^{*} X$ such that $\rho_{j}$, together with $\tilde{\eta}, x$ and $\underline{\hat{\xi}}$, are local coordinates on $\tilde{\mathcal{U}}$; this holds if $\tilde{\vartheta}_{0}$ is identified with a subset of $\mathscr{G}^{\mathrm{b}} S_{Y}^{*} X$ and $\tilde{U}$ is a product neighborhood of this in ${ }^{\mathrm{b}} S^{*} X$ in terms of the coordinates (6-1). 
Note that since $\underline{\hat{\xi}}=0$ on $\dot{\Sigma} \cap{ }^{\mathrm{b}} S_{Y}^{*} X$, for points $q$ in $\dot{\Sigma}$ one can ensure that $\underline{\xi}$ is small by ensuring that $\tilde{\pi}(q)$ is close to $q_{0}$ and $x(q)$ is small; see the discussion around (8-5) and after (8-7). By reducing $\tilde{\mathcal{U}}$ if needed (this keeps all previously discussed properties), we may also assume that it is disjoint from $\mathrm{WF}_{\mathrm{b}}^{-1, \infty}(f)$.

Hence,

$$
\left|\underline{\zeta}_{n-1}\right|^{-1} W_{0} \rho_{j}=\sum_{i=1}^{2 n-4} \tilde{F}_{j i} \rho_{i}+\tilde{F}_{j, 2 n-3} \tilde{\eta} \quad \text { for } j=2, \ldots, 2 n-4,
$$

with $\tilde{F}_{j i}$ smooth for $i=1, \ldots, 2 n-3$ and $j=2, \ldots, 2 n-4$. Then we extend $\rho_{j}$ to a function on ${ }^{\mathrm{b}} T^{*} X \backslash o$ (using the coordinates $(x, y, \underline{\xi}, \underline{\zeta})$ ), and conclude that

$$
\left|\underline{\zeta}_{n-1}\right|^{-1} \mathrm{H}_{\tilde{p}} \rho_{j}=\sum_{l=1}^{2 n-4} \tilde{F}_{j l} \rho_{l}+\tilde{F}_{j, 2 n-3} \tilde{\eta}+\tilde{F}_{j 0} x \quad \text { for } j=2, \ldots, 2 n-4,
$$

with $\tilde{F}_{j l}$ smooth. Similarly, with $\check{F}_{l}$ smooth,

$$
\left|\underline{\zeta}_{n-1}\right|^{-1} \mathrm{H}_{\tilde{p}} \tilde{\eta}=2+\sum_{l=1}^{2 n-4} \check{F}_{l} \rho_{l}+\check{F}_{2 n-3} \tilde{\eta}+\check{F}_{0} x .
$$

Let

$$
\omega=|x|^{2}+\sum_{j=1}^{2 n-4} \rho_{j}^{2}
$$

Finally, we let

$$
\phi=\tilde{\eta}+\omega /\left(\epsilon^{2} \delta\right)
$$

and define $a$ by

$$
a=\chi_{0}\left(\digamma^{-1}(2-\phi / \delta)\right) \chi_{1}((\tilde{\eta} \delta) / \epsilon \delta+1) \chi_{2}\left(|\underline{\xi}|^{2} / \underline{\zeta}_{n-1}^{2}\right),
$$

with $\chi_{0}, \chi_{1}$ and $\chi_{2}$ as in the case of the normal propagation estimate, stated after (8-7). We always assume $\epsilon<1$, so we have

$$
\phi \leq 2 \delta \text { and } \tilde{\eta} \geq-\epsilon \delta-\delta \geq-2 \delta \text { on } \operatorname{supp} a .
$$

Since $\omega \geq 0$, the first of these inequalities implies that $\tilde{\eta} \leq 2 \delta$, so

$$
|\tilde{\eta}| \leq 2 \delta \quad \text { on } \operatorname{supp} a .
$$

Hence,

$$
\omega \leq \epsilon^{2} \delta(2 \delta-\tilde{\eta}) \leq 4 \delta^{2} \epsilon^{2} .
$$

Thus, supp $a$ lies in $\tilde{U}$ for $\delta>0$ sufficiently small. Moreover,

$$
\tilde{\eta} \in[-\delta-\epsilon \delta,-\delta] \text { and } \omega^{1 / 2} \leq 2 \epsilon \delta \text { on } \operatorname{supp} d \chi_{1},
$$

so this region lies in (8-32) after $\epsilon$ and $\delta$ are both replaced by appropriate constant multiples, namely the present $\delta$ should be replaced by $\delta /\left(2\left|\left(\underline{\zeta}_{n-1}\right)_{0}\right|\right)$. 
We proceed as in the case of hyperbolic points, letting $A_{0} \in \Psi_{\mathrm{b}}^{0}(X)$ with $\sigma_{\mathrm{b}, 0}\left(A_{0}\right)=a$, supported in the coordinate chart. Also let $\Lambda_{r}$ be scalar, with symbol

$$
\left|\underline{\zeta}_{n-1}\right|^{s+1 / 2}\left(1+r\left|\underline{\zeta}_{n-1}\right|^{2}\right)^{-s} \text { Id } \quad \text { for } r \in[0,1),
$$

so $A_{r}=A \Lambda_{r} \in \Psi_{\mathrm{b}}^{0}(X)$ for $r>0$ and it is uniformly bounded in $\Psi_{\mathrm{bc}}^{s+1 / 2}(X)$. Then, for $r>0$,

$$
\begin{aligned}
\left\langle\imath A_{r}^{*} A_{r} P u, u\right\rangle-\left\langle\imath A_{r}^{*} A_{r} u, P u\right\rangle & =\left\langle\imath\left[A_{r}^{*} A_{r}, P\right] u, u\right\rangle+\left\langle\imath\left(P-P^{*}\right) A_{r}^{*} A_{r} u, u\right\rangle \\
& =\left\langle\imath\left[A_{r}^{*} A_{r}, P\right] u, u\right\rangle-2 \operatorname{Im} \lambda\left\|A_{r} u\right\|^{2} .
\end{aligned}
$$

and we compute the commutator here using Proposition 8.1. We arrange the terms of the proposition so that the terms in which a vector field differentiates $\chi_{1}$ are included in $E_{r}$ and the terms in which a vector fields differentiates $\chi_{2}$ are included in $E_{r}^{\prime}$. Thus, we have

$$
\imath A_{r}^{*} A_{r} P-\imath P A_{r}^{*} A_{r}=\left(x D_{x}\right)^{*} C_{r}^{\sharp}\left(x D_{x}\right)+\left(x D_{x}\right)^{*} x C_{r}^{\prime}+x C_{r}^{\prime \prime}\left(x D_{x}\right)+x^{2} C_{r}^{b}+E_{r}+E_{r}^{\prime}+F_{r},
$$

with

$$
\begin{aligned}
\sigma_{\mathrm{b}, 2 s}\left(C_{r}^{\sharp}\right) & =w_{r}^{2}\left(\digamma^{-1} \delta^{-1} a\left|\underline{\zeta}_{n-1}\right|^{-1}\left(\hat{f}^{\sharp}+\epsilon^{-2} \delta^{-1} f^{\sharp}\right) \chi_{0}^{\prime} \chi_{1} \chi_{2}+a^{2} \tilde{c}_{r}^{\sharp}\right), \\
\sigma_{\mathrm{b}, 2 s+1}\left(C_{r}^{\prime}\right) & =w_{r}^{2}\left(\digamma^{-1} \delta^{-1} a\left(\hat{f}^{\prime}+\delta^{-1} \epsilon^{-2} f^{\prime}\right) \chi_{0}^{\prime} \chi_{1} \chi_{2}+a^{2} \tilde{c}_{r}^{\prime}\right), \\
\sigma_{\mathrm{b}, 2 s+1}\left(C_{r}^{\prime \prime}\right) & =w_{r}^{2}\left(\digamma^{-1} \delta^{-1} a\left(\hat{f}^{\prime \prime}+\delta^{-1} \epsilon^{-2} f^{\prime \prime}\right) \chi_{0}^{\prime} \chi_{1} \chi_{2}+a^{2} \tilde{c}_{r}^{\prime \prime}\right), \\
\sigma_{\mathrm{b}, 2 s+2}\left(C_{r}^{b}\right) & =w_{r}^{2}\left(\digamma^{-1} \delta^{-1}\left|\underline{\zeta}_{n-1}\right| a\left(4+\hat{f}^{b}+\delta^{-1} \epsilon^{-2} f^{b}\right) \chi_{0}^{\prime} \chi_{1} \chi_{2}+a^{2} \tilde{c}_{r}^{b}\right),
\end{aligned}
$$

where $f^{\sharp}, f^{\prime}, f^{\prime \prime}$ and $f^{\mathrm{b}}$ as well as $\hat{f}^{\sharp}, \hat{f}^{\prime}, \hat{f}^{\prime \prime}$ and $\hat{f}^{\mathrm{b}}$ are all smooth functions on ${ }^{\mathrm{b}} T^{*} X \backslash o$, homogeneous of degree 0 (and independent of $\epsilon$ and $\delta$ ). Moreover, $f^{\sharp}, f^{\prime}, f^{\prime \prime}, f^{b}$ arise when $\omega$ is differentiated in $\chi_{0}\left(\digamma^{-1}(2-\phi / \delta)\right)$, while $\hat{f}^{\sharp}, \hat{f}^{\prime}, \hat{f}^{\prime \prime}$ and $\hat{f}^{b}$ arise when $\tilde{\eta}$ is differentiated in $\chi_{0}\left(\digamma^{-1}(2-\phi / \delta)\right)$, and comprise all such terms with the exception of part of that arising from the $-\mathrm{H}_{h}$ component of $\left.V^{\mathrm{b}}\right|_{Y}$ (which gives the 4 on the last line above, modulo a term included in $\hat{f}^{b}$ and vanishing $\omega=0$ ). In addition, since $V^{\bullet} \rho^{2}=2 \rho V^{\bullet} \rho$ for any function $\rho$, the terms $f^{\bullet}$ for $\bullet=\sharp,,^{\prime}$, , , b have vanishing factors of $\rho_{l}$ and $x$, with the structure of the remaining factor dictated by the form of $V^{\bullet} \rho_{l}$ and $V^{*} x$, respectively. Thus, using (8-34) to compute $f^{b}$, (8-35) to compute $\hat{f}^{b}$, we have

$$
\begin{aligned}
& f^{\sharp}=\sum_{k} \rho_{k} f_{k}^{\sharp}+x f_{0}^{\sharp}, \\
& f^{\mathrm{b}}=\sum_{k l} \rho_{k} \rho_{l} f_{k l}^{\mathrm{b}}+\sum_{k} \rho_{k} x f_{k}^{\mathrm{b}}+x^{2} f_{0}+\sum_{k} \rho_{k} \tilde{\eta} f_{k+}^{\mathrm{b}}, \\
& f^{\bullet}=\sum_{k} \rho_{k} f_{k}^{\bullet}+x f_{0}^{\bullet} \quad \text { for } \bullet=^{\prime},{ }^{\prime \prime}, \quad \hat{f}^{b}=x \hat{f}_{0}^{b}+\sum_{k} \rho_{k} \hat{f}_{k}^{b}+\tilde{\eta} \hat{f}_{+}^{b},
\end{aligned}
$$

with $f_{k}^{\sharp}$ etc. smooth. We deduce that

$$
\begin{array}{llrl}
\epsilon^{-2} \delta^{-1}\left|f^{\sharp}\right| \leq C \epsilon^{-1}, & & \left|\hat{f}^{\sharp}\right| \leq C, \\
\epsilon^{-2} \delta^{-1}\left|f^{\bullet}\right| \leq C \epsilon^{-1}, & & \left|\hat{f}^{\bullet}\right| \leq C \quad \text { for } \bullet=^{\prime},,^{\prime \prime}, \\
\epsilon^{-2} \delta^{-1}\left|f^{b}\right| \leq C \epsilon^{-1} \delta, & & \left|\hat{f}^{b}\right| \leq C \delta .
\end{array}
$$

We remark that although thus far we worked with a single $q_{0} \in K$, the same construction works with $q_{0}$ in a neighborhood ${ }_{q_{0}^{\prime}}$ of a fixed $q_{0}^{\prime} \in K$, with a uniform constant $C$. In view of the compactness 
of $K$, this suffices (by the rest of the argument we present below) to give the uniform estimate of the proposition.

Since (8-46)-(8-48) are exactly the same (with slightly different notation) as (6.16)-(6.18) of [Vasy 2010a], the rest of the proof is analogous, except that [Vasy 2010a, Lemma 4.6] is replaced by Lemma 8.4 here. Thus, for a small constant $c_{0}>0$ to be determined, which we may assume to be less than $C$, we demand below that the expressions on the right sides of (8-46) are bounded by $c_{0}(\epsilon \delta)^{-1}$, those on the right sides of (8-47) are bounded by $c_{0}(\epsilon \delta)^{-1 / 2}$, and those on the right sides of (8-48) are bounded by $c_{0}$. This demand is due to the appearance of two, one, and zero, respectively, factors of $x D_{x}$ in (8-44) for the terms whose principal symbols are affected by these, taking into account that in view of Lemma 8.4 we can estimate $\left\|Q_{i} v\right\|$ by $C_{\mathscr{G}_{, K}}(\epsilon \delta)^{1 / 2}\left\|D_{y_{n-1}} v\right\|$ if $v$ is microlocalized to a $\epsilon \delta$-neighborhood of $\mathscr{G}$, which is the case for us with $v=A_{r} u$ in terms of support properties of $a$.

Thus, recalling that $c_{0}>0$ is to be determined, we require that

$$
\left(C / c_{0}\right)^{2} \delta \leq \epsilon \leq 1
$$

and

$$
\delta<\left(c_{0} / C\right)^{2}
$$

see [Vasy 2010a, Proposition 6.1] for motivation. Then with $\epsilon, \delta$ satisfying (8-49) and (8-50) and hence $\delta^{-1}>\left(C / c_{0}\right)^{2}>C / c_{0}$, the bounds (8-46)-(8-48) give that

$$
\begin{array}{lll}
\epsilon^{-2} \delta^{-1}\left|f^{\sharp}\right| \leq c_{0} \delta^{-1} \epsilon^{-1}, & & \left|\hat{f}^{\sharp}\right| \leq c_{0} \delta^{-1} \epsilon^{-1}, \\
\epsilon^{-2} \delta^{-1}\left|f^{\bullet}\right| \leq c_{0} \delta^{-1 / 2} \epsilon^{-1 / 2}, & & \left|\hat{f}^{\bullet}\right| \leq c_{0} \delta^{-1 / 2} \epsilon^{-1 / 2} \quad \text { for } \bullet^{-=^{\prime},,^{\prime \prime}} \\
\epsilon^{-2} \delta^{-1}\left|f^{\mathrm{b}}\right| \leq c_{0}, & & \left|\hat{f}^{\mathrm{b}}\right| \leq c_{0},
\end{array}
$$

as desired. One deduces that

$$
\begin{aligned}
& \imath A_{r}^{*} A_{r} P-\imath P A_{r}^{*} A_{r} \\
& \quad=\tilde{B}_{r}^{*}\left(C^{*} x^{2} C+x R^{\mathrm{b}} x+\left(x D_{x}\right)^{*} \tilde{R}^{\prime} x+x \tilde{R}^{\prime \prime}\left(x D_{x}\right)+\left(x D_{x}\right)^{*} R^{\sharp}\left(x D_{x}\right)\right) \tilde{B}_{r}+R_{r}^{\prime \prime}+E_{r}+E_{r}^{\prime}
\end{aligned}
$$

with

$$
\begin{gathered}
R^{\mathrm{b}} \in \Psi_{\mathrm{b}}^{0}(X), \quad \tilde{R}^{\prime}, \tilde{R}^{\prime \prime} \in \Psi_{\mathrm{b}}^{-1}(X), \quad R^{\sharp} \in \Psi_{\mathrm{b}}^{-2}(X), \\
R_{r}^{\prime \prime} \in L^{\infty}\left((0,1) ; \operatorname{Diff}_{0}^{2} \Psi_{\mathrm{b}}^{2 s-1}(X)\right), \quad E_{r}, E_{r}^{\prime} \in L^{\infty}\left((0,1) ; \operatorname{Diff}_{0}^{2} \Psi_{\mathrm{b}}^{2 s}(X)\right),
\end{gathered}
$$

with

$$
\mathrm{WF}_{\mathrm{b}}^{\prime}(E) \subset \tilde{\eta}^{-1}((-\delta-\epsilon \delta,-\delta]) \cap \omega^{-1}\left(\left[0,4 \delta^{2} \epsilon^{2}\right)\right) \subset \tilde{u}
$$

(see (8-41)), $\mathrm{WF}_{\mathrm{b}}^{\prime}\left(E^{\prime}\right) \cap \dot{\Sigma}=\varnothing$, and with $r^{\mathrm{b}}=\sigma_{\mathrm{b}, 0}\left(R^{\mathrm{b}}\right), \tilde{r}^{\prime}=\sigma_{\mathrm{b},-1}\left(\tilde{R}^{\prime}\right), \tilde{r}^{\prime \prime}=\sigma_{\mathrm{b},-1}\left(\tilde{R}^{\prime \prime}\right), r^{\sharp} \in \sigma_{\mathrm{b},-2}\left(R^{\sharp}\right)$,

$$
\begin{aligned}
\left|r^{\mathrm{b}}\right| & \leq 2 c_{0}+C_{2} \delta \digamma^{-1}, & & \left|\underline{\zeta}_{n-1} \tilde{r}^{\prime}\right| \leq 2 c_{0} \delta^{-1 / 2} \epsilon^{-1 / 2}+C_{2} \delta \digamma^{-1}, \\
\left|\underline{\zeta}_{n-1} \tilde{r}^{\prime \prime}\right| & \leq 2 c_{0} \delta^{-1 / 2} \epsilon^{-1 / 2}+C_{2} \delta \digamma^{-1}, & \left|\underline{\zeta}_{n-1}^{2} r^{\sharp}\right| & \leq 2 c_{0} \delta^{-1} \epsilon^{-1}+C_{2} \delta \digamma^{-1} .
\end{aligned}
$$


These are analogues of the result of the second displayed equation after [Vasy 2008c, Equation (7.16)], as corrected in [Vasy 2008a], with the small (at this point arbitrary) constant $c_{0}$ replacing some constants given there in terms of $\epsilon$ and $\delta$; see [Vasy 2010a, Equation (6.25)] for estimates stated in exactly the same form in the form-valued setting. The rest of the argument proceeds as in the proof of [Vasy 2008c, Proposition 7.3], taking into account [Vasy 2008a], and using Lemma 8.4 in place of [Vasy 2008c, Lemma 7.1].

Since for $\lambda$ real, $\lambda<(n-1)^{2} / 4$, both forward and backward propagation are covered by these two results (see Remarks 8.3 and 8.7), we deduce our main result on the propagation of singularities:

Theorem 8.8. Suppose that $P=\square+\lambda$, with $\lambda<(n-1)^{2} / 4$, for $m \in \mathbb{R}$ or $m=\infty$. Suppose $u \in H_{0, \mathrm{~b}, \text { loc }}^{1, k}(X)$ for some $k \leq 0$. Then

$$
\left(\mathrm{WF}_{\mathrm{b}}^{1, m}(u) \cap \dot{\Sigma}\right) \backslash \mathrm{WF}_{\mathrm{b}}^{-1, m+1}(P u)
$$

is a union of maximally extended generalized broken bicharacteristics of the conformal metric $\hat{g}$ in

$$
\dot{\Sigma} \backslash \mathrm{WF}_{\mathrm{b}}^{-1, m+1}(P u) .
$$

In particular, if $P u=0$, then $\mathrm{WF}_{\mathrm{b}}^{1, \infty}(u) \subset \dot{\Sigma}$ is a union of maximally extended generalized broken bicharacteristics of $\hat{g}$.

Proof. The proof proceeds as that of [Vasy 2008c, Theorem 8.1], since Propositions 8.2 and 8.6 are complete analogues of [Vasy 2008c, Propositions 6.2 and 7.3]. Given the results of the preceding sections of [Vasy 2008c], the argument proving [Vasy 2008c, Theorem 8.1] is itself only a slight modification of an argument originally due to Melrose and Sjöstrand [1978], as presented by Lebeau [1997] (although we do not need Lebeau's treatment of corners here).

For the convenience of the reader we give a very sketchy version of the proof. To start with, propagation of singularities has already been proved in $X^{\circ}$; this is the theorem of Duistermaat and Hörmander [Hörmander 1971]. Now, the theorem can easily be localized — the global version follows by a Zorn's lemma argument; see [Vasy 2008c, proof of Theorem 8.1] for details. Indeed, in view of the Duistermaat and Hörmander's result, it suffices to show that if

$$
q_{0} \in \mathrm{WF}_{\mathrm{b}}^{1, m}(u) \backslash \mathrm{WF}_{\mathrm{b}}^{-1, m+1}(P u) \quad \text { and } \quad q_{0} \in{ }^{\mathrm{b}} T_{Y}^{*} X,
$$

then

there exists a generalized broken bicharacteristic $\gamma:\left[-\epsilon_{0}, 0\right] \rightarrow \dot{\Sigma}$,

with $\epsilon_{0}>0, \quad \gamma(0)=q_{0}, \quad \gamma(s) \in \mathrm{WF}_{\mathrm{b}}^{1, m}(u) \backslash \mathrm{WF}_{\mathrm{b}}^{-1, m+1}(P u), \quad s \in\left[-\epsilon_{0}, 0\right]$,

for the existence of a GBB on $\left[0, \epsilon_{0}\right]$ can be demonstrated similarly by replacing the forward propagation estimates by backward ones, and, directly from Definition 1.1, piecing together the two GBBs gives one defined on $\left[-\epsilon_{0}, \epsilon_{0}\right]$. Note that (8-55) implies that $q_{0} \in \mathscr{G} \cup \mathscr{H}$ by microlocal elliptic regularity, Proposition 7.7.

Now suppose $q_{0} \in\left(\mathrm{WF}_{\mathrm{b}}^{1, m}(u) \backslash \mathrm{WF}_{\mathrm{b}}^{-1, m+1}(P u)\right) \cap{ }^{\mathrm{b}} T_{Y}^{*} X \cap \mathcal{H}$. We use the notation of Proposition 8.2. Then $\gamma$ in (8-55) is constructed by taking a sequence $q_{n} \rightarrow q_{0}$, where $q_{n} \in T^{*} X^{\circ}$ and $\eta\left(q_{n}\right)=-\hat{\xi}\left(q_{n}\right)<0$ 
and GBB $\gamma_{n}:\left[-\epsilon_{0}, 0\right] \rightarrow \dot{\Sigma}$ with $\gamma_{n}(0)=q_{n}$ and with $\gamma_{n}(s) \in\left(\mathrm{WF}_{\mathrm{b}}^{1, m}(u) \backslash \mathrm{WF}_{\mathrm{b}}^{-1, m+1}(P u)\right) \cap T^{*} X^{\circ}$ for $s \in\left[-\epsilon_{0}, 0\right]$. Once this is done, by compactness of GBB with image in a compact set (see [Vasy 2008c, Proposition 5.5] and [Lebeau 1997, Proposition 6]), one can extract a uniformly convergent subsequence, converging to some $\gamma$, giving (8-56). Now, the $q_{n}$ arise directly from Proposition 8.2, by shrinking $U$ (via shrinking $\delta$ in (8-5)); namely under our assumption on $q_{0}$, for each such $U$ there must exist a $q \in \mathrm{WF}_{\mathrm{b}}^{1, m}(u)$ in $U \cap\{\eta<0\}$. The $\gamma_{n}$ then arise from the theorem of Duistermaat and Hörmander, using that $\eta\left(q_{n}\right)<0$ implies that the backward GBB from $q_{n}$ cannot meet $Y$ for some time $\epsilon_{0}$, uniform in $n$ - this is essentially due to $\eta$ being strictly increasing along GBB microlocally, and $\eta$ vanishing at $\dot{\Sigma} \cap{ }^{\mathrm{b}} T_{Y}^{*} X$ : So as long as $\eta$ is negative, the GBB cannot hit the boundary. For more details, see the proof of [Vasy 2008c, Theorem 8.1].

Finally, suppose $q_{0} \in\left(\mathrm{WF}_{\mathrm{b}}^{1, m}(u) \backslash \mathrm{WF}_{\mathrm{b}}^{-1, m+1}(P u)\right) \cap{ }^{\mathrm{b}} T_{Y}^{*} X \cap G$, which is the more technical case. This part of the argument is present in essentially the same form in [Melrose and Sjöstrand 1978]. Lebeau [1997, Proposition VII.1] gives a very nice presentation; see the proof of [Vasy 2008c, Theorem 8.1] for an overview with more details. The rough idea for constructing the GBB $\gamma$ for (8-56) is to define approximations to it using Proposition 8.6. First, recall that in Proposition 8.6, applied at $q_{0}, W_{0}$ is the coordinate projection (push forward) of $\mathrm{H}_{p}$, evaluated at $\hat{\pi}^{-1}\left(q_{0}\right)$, to $T^{*} Y$. Thus, one should think of the point $\tilde{\pi}\left(q_{0}\right)-\delta W_{0}$ in $T^{*} Y$ as an $O\left(\delta^{2}\right)$ approximation of where a backward GBB should be after "time" (that is, parameter value) $\delta$. This is used as follows: Given $\delta>0$, Proposition 8.6 gives the existence of a point $q_{1}$ in $\mathrm{WF}_{\mathrm{b}}^{1, m}(u)$ that is, roughly speaking, $O\left(\delta^{2}\right)$ from $\tilde{\pi}\left(q_{1}\right)-\left(\tilde{\pi}\left(q_{0}\right)-\delta W_{0}\right)$, with $x\left(q_{1}\right)$ being $\mathcal{O}\left(\delta^{2}\right)$ as well. Then, from $q_{1}$, one can repeat this procedure (replacing $q_{0}$ by $q_{1}$ in Proposition 8.6) there are some technical issues corresponding to $q_{1}$ being in the boundary or not, and also whether in the former case the backward GBB hits the boundary in time $\delta$. Taking $\delta=2^{-N} \epsilon_{0}$, this gives $2^{N}+1$ points $q_{j}$ corresponding to the dyadic points on the parameter interval $\left[-\epsilon_{0}, 0\right]$. It is helpful to consider this as analogous to a discrete approximation of solving an ODE without the presence of the boundary by taking steps of size $2^{-N} \epsilon_{0}$. Defining $\gamma_{N}(s)$ for only these dyadic values, one can then get a subsequence $\gamma_{N_{k}}$ that converges, as $k \rightarrow \infty$, at $s=2^{-n} j \epsilon_{0}$ for all $n \geq 1$ and $0 \leq j \leq 2^{n}$ integers. (Note that $\gamma_{N_{k}}(s)$ is defined for these values of $s$ for $k$ sufficiently large!) One then checks as in Lebeau's proof that the result is the restriction of a GBB to dyadic parameter values. Again, we refer to [Lebeau 1997, Proposition VII.1] and the proof of [Vasy 2008c, Theorem 8.1] for more details.

In fact, even if $\operatorname{Im} \lambda \neq 0$, we get one-sided statements:

Theorem 8.9. Suppose that $P=\square+\lambda$ and $\operatorname{Im} \lambda>0$, and $m \in \mathbb{R}$ or $m=\infty$. Suppose $u \in H_{0, \mathrm{~b}, \mathrm{loc}}^{1, k}(X)$ for some $k \leq 0$. Then

$$
\left(\mathrm{WF}_{\mathrm{b}}^{1, m}(u) \cap \dot{\Sigma}\right) \backslash \mathrm{WF}_{\mathrm{b}}^{-1, m+1}(P u)
$$

is a union of maximally forward extended (and in the case $\operatorname{Im} \lambda<0$ backward extended) generalized broken bicharacteristics of the conformal metric $\hat{g}$ in

$$
\dot{\Sigma} \backslash \mathrm{WF}_{\mathrm{b}}^{-1, m+1}(P u) \text {. }
$$


In particular, if $P u=0$, then $\mathrm{WF}_{\mathrm{b}}^{1, \infty}(u) \subset \dot{\Sigma}$ is a union of maximally extended generalized broken bicharacteristics of $\hat{g}$.

Proof. The proof proceeds again as for Theorem 8.8, but now Propositions 8.2 and 8.6 only allow propagation in one direction. Thus, if $\operatorname{Im} \lambda<0$, they allow one to conclude that if a point in $\dot{\Sigma} \backslash$ $\mathrm{WF}_{\mathrm{b}}^{-1, m+1}(P u)$ is in $\mathrm{WF}_{\mathrm{b}}^{1, m}(u)$, then there is another point in $\mathrm{WF}_{\mathrm{b}}^{1, m}(u)$ that is roughly along a backward GBB segment emanating from it. Then an actual backward GBB can be constructed as in [Melrose and Sjöstrand 1978; Lebeau 1997].

In the absence of b-wave front set we can easily read off the actual expansion at the boundary as well.

Proposition 8.10. Suppose that $P=\square+\lambda$, where $\lambda \in \mathbb{C}$. Let

$$
s_{ \pm}(\lambda)=\frac{1}{2}(n-1) \pm \sqrt{\frac{1}{4}(n-1)^{2}-\lambda} .
$$

Suppose $u \in H_{0, \mathrm{loc}}^{1}(X), \mathrm{WF}_{\mathrm{b}}^{1, \infty}(u)=\varnothing$ and $P u \in \dot{\mathscr{C}}^{\infty}(X)$. Then

$$
u=x^{s_{+}(\lambda)} v_{+} \quad \text { and } \quad v_{+} \in \mathscr{C}^{\infty}(X)
$$

Conversely, if $\lambda<(n-1)^{2} / 4$, given any $g_{+} \in \mathscr{C}^{\infty}(Y)$, there exists $v_{+} \in \mathscr{C}^{\infty}(X)$ and $\left.v_{+}\right|_{Y}=g_{+}$such that $u=x^{s_{+}(\lambda)} v_{+}$satisfies $P u \in \dot{\mathscr{C}}^{\infty}(X)$; in particular $u \in H_{0, \mathrm{loc}}^{1}(X)$ and $\mathrm{WF}_{\mathrm{b}}^{1, \infty}(u)=\varnothing$.

This proposition reiterates the importance of the constraint on $\lambda$ in that

$$
x^{(n-1) / 2+i \alpha} \notin H_{0, \text { loc }}^{1}(X) \quad \text { for } \alpha \in \mathbb{R} ;
$$

for $\lambda \geq(n-1)^{2} / 4$, the growth or decay relative to $H_{0, \text { loc }}^{1}(X)$ does not distinguish between the two approximate solutions $x^{s_{ \pm}(\lambda)} v_{ \pm}$having $v_{ \pm} \in \mathscr{C}^{\infty}(X)$.

Proof. For the first part of the lemma, by Lemma 5.16 and the remark after, we have $u \in \mathscr{A}^{(n-1) / 2}(X)$ under our assumptions. By (7-1),

$$
P+\left(\left(x D_{x}+\imath(n-1)\right)\left(x D_{x}\right)-\lambda\right) \in x \operatorname{Diff}_{\mathrm{b}}^{2}(X) .
$$

This is, up to a change in overall the sign of the second summand,

$$
\left(x D_{x}+\imath(n-1)\right)\left(x D_{x}\right)-\lambda,
$$

the same as the analogous expression in the de Sitter setting; see the first line of the proof of [Vasy 2010b, Lemma 4.13]. Thus, the proof of that lemma goes through without changes — the reader needs to keep in mind that $u \in \mathscr{A}^{(n-1) / 2}(X)$ excludes one of the indicial roots from appearing in the argument of that lemma. (In the de Sitter setting, in [Vasy 2010b, Lemma 4.13] there was no a priori weight, relative to which one has conormality, specified.)

The converse again works as in [Vasy 2010b, Lemma 4.13] using (8-58).

We can now state the "inhomogeneous Dirichlet problem": 
Theorem 8.11. Assume $(T F)$ and $(P T)$. Suppose $\lambda<(n-1)^{2} / 4$, and $s_{+}(\lambda)-s_{-}(\lambda)=2 \sqrt{(n-1)^{2} / 4-\lambda}$ is not an integer, and $P=P(\lambda)=\square_{g}+\lambda$.

Given $v_{0} \in \mathscr{C}^{\infty}(Y)$ and $f \in \dot{\mathscr{C}}^{\infty}(X)$, both supported in $\left\{t \geq t_{0}\right\}$, the problem

$$
P u=f,\left.\quad u\right|_{t<t_{0}}=0, \quad u=x^{s_{-}(\lambda)} v_{-}+x^{s_{+}(\lambda)} v_{+}, \quad v_{ \pm} \in \mathscr{C}^{\infty}(X),\left.\quad v_{-}\right|_{Y}=v_{0},
$$

has a unique solution

If $s_{+}(\lambda)-s_{-}(\lambda)$ is an integer, the same conclusion holds if we replace the condition $v_{-} \in \mathscr{C}^{\infty}(X)$ by $v_{-} \in \mathscr{C}^{\infty}(X)+x^{s_{+}(\lambda)-s_{-}(\lambda)} \log x \mathscr{C}^{\infty}(X)$.

Proof. The proof of [Vasy 2010b, Lemma 4.13] shows that there exists $\tilde{u}$, supported in $t \geq t_{0}$, such that $\tilde{u}=x^{s_{-}(\lambda)} v_{-}, \quad v_{-}$is as in the statement of the theorem, and $P \tilde{u} \in \dot{\mathscr{C}}^{\infty}(X)$. Now let $u^{\prime}$ be the solution of $P u^{\prime}=f-P \tilde{u}$ supported in $\left\{t \geq t_{0}\right\}$, whose existence follows from Theorem 4.16, and which is of the form $x^{s_{+}(\lambda)} v_{+}$by Theorem 8.8 and Proposition 8.10. Then $u=\tilde{u}+u^{\prime}$ solves the PDE as stated. Uniqueness follows from the basic well-posedness theorem, Theorem 4.16.

Finally we add well-posedness of possibly rough initial data:

Theorem 8.12. Assume $(T F)$ and $(P T)$. Suppose $f \in H_{0, b, l o c}^{-1, m+1}(X)$ for some $m \in \mathbb{R}$, and let $m^{\prime} \leq m$. Then (1-6) has a unique solution in $H_{0, \mathrm{~b}, \mathrm{loc}}^{1, m^{\prime}}(X)$, which in fact lies in $H_{0, \mathrm{~b}, \mathrm{loc}}^{1, m}(X)$, and for all compact $K \subset X$ there exists a compact $K^{\prime} \subset X$ and a constant $C>0$ such that

$$
\|u\|_{H_{0}^{1, m}(K)} \leq C\|f\|_{H_{0, \mathrm{~b}}^{-1, m+1}\left(K^{\prime}\right)} \cdot
$$

Remark 8.13. It should be emphasized that if one only wants to prove this result, without microlocal propagation, one could use more elementary energy estimates.

Proof. If $m \geq 0$, then by Theorem 4.16, (1-6) has a unique solution in $H_{0, \text { loc }}^{1}(X)$, and by propagation of singularities it lies in $H_{0, \mathrm{~b}, \mathrm{loc}}^{1, m}(X)$, with the desired estimate. Moreover, again by the propagation of singularities, any solution of $(1-6)$ in $H_{0, \mathrm{~b}, \mathrm{loc}}^{1, m^{\prime}}(X)$ lies in $H_{0, \mathrm{~b}, \mathrm{loc}}^{1, m}(X)$, so the solution is indeed unique even in $H_{0, \mathrm{~b}, \mathrm{loc}}^{1, m^{\prime}}(X)$.

If $m<0$, uniqueness and the stability estimate follow as above. To see existence, let $T_{0}<t_{0}$, and let $f_{j} \rightarrow f$ such that $f_{j} \in H_{0, \mathrm{~b}, \mathrm{loc}}^{-1,1}$ and supp $f_{j} \subset\left\{t>T_{0}\right\}$. This can be achieved by taking $A_{r} \in \Psi_{\mathrm{bc}}^{-\infty}(X)$ with properly supported Schwartz kernel (of sufficiently small support) such that $\left\{A_{r}: r \in(0,1]\right\}$ is a bounded family in $\Psi_{\mathrm{bc}}^{0}(X)$, converging to Id in $\Psi_{\mathrm{bc}}^{\epsilon}(X)$ for $\epsilon>0$; then with $f_{j}=A_{r_{j}} f, r_{j} \rightarrow 0$, we have the desired properties. By Theorem 4.16, (1-6) with $f$ replaced by $f_{j}$ has a unique solution $u_{j} \in H_{0, \operatorname{loc}}^{1}(X)$. Moreover, by the propagation of singularities, one has a uniform estimate

$$
\left\|u_{k}-u_{j}\right\|_{H_{0}^{1, m}(K)} \leq C\left\|f_{k}-f_{j}\right\|_{H_{0, \mathrm{~b}}^{-1, m+1}\left(K^{\prime}\right)},
$$

with $C$ independent of $j$ and $k$. In view of the convergence of the $f_{j}$ in $H_{0, \mathrm{~b}}^{-1, m+1}\left(K^{\prime}\right)$, we deduce the convergence of the $u_{j}$ in $H_{0, \mathrm{~b}}^{1, m}(K)$ to some $u \in H_{0, \mathrm{~b}}^{1, m}(K)$; hence (by uniqueness) we deduce the existence of $u \in H_{0, \mathrm{~b}, \mathrm{loc}}^{1, m}(X)$ solving $P u=f$ with support in $\left\{t \geq T_{0}\right\}$. However, as supp $f \subset\left\{t \geq t_{0}\right\}$, uniqueness shows the vanishing of $u$ on $\left\{t<t_{0}\right\}$, proving the theorem. 


\section{Acknowledgments}

I am very grateful to Dean Baskin, Rafe Mazzeo and Richard Melrose for helpful discussions. I would also like to thank the careful referee whose comments helped to improve the exposition significantly and also led to the removal of some very confusing typos.

\section{References}

[Anderson 2004] M. T. Anderson, "On the structure of asymptotically de Sitter and anti-de Sitter spaces", Adv. Theor. Math. Phys. 8:5 (2004), 861-894. MR 2006i:53102 Zbl 1096.83018

[Anderson 2005] M. T. Anderson, "Existence and stability of even-dimensional asymptotically de Sitter spaces", Ann. Henri Poincaré 6:5 (2005), 801-820. MR 2007c:53090 Zbl 1100.83004

[Anderson 2008] M. T. Anderson, "Einstein metrics with prescribed conformal infinity on 4-manifolds", Geom. Funct. Anal. 18:2 (2008), 305-366. MR 2009f:53061 Zbl 1148.53033

[Anderson and Chruściel 2005] M. T. Anderson and P. T. Chruściel, "Asymptotically simple solutions of the vacuum Einstein equations in even dimensions", Comm. Math. Phys. 260:3 (2005), 557-577. MR 2007b:58041 Zbl 1094.83002

[Bachelot 2008] A. Bachelot, "The Dirac system on the anti-de Sitter universe", Comm. Math. Phys. 283:1 (2008), 127-167. MR 2009f:83029 Zbl 1153.83013

[Baskin 2010] D. Baskin, "A parametrix for the fundamental solution of the Klein-Gordon equation on asymptotically de Sitter spaces”, J. Funct. Anal. 259:7 (2010), 1673-1719. MR 2011h:58044 Zbl 1200.35247

[Bony and Häfner 2008] J.-F. Bony and D. Häfner, "Decay and non-decay of the local energy for the wave equation on the de Sitter-Schwarzschild metric", Comm. Math. Phys. 282:3 (2008), 697-719. MR 2010h:58041 Zbl 1159.35007

[Breitenlohner and Freedman 1982a] P. Breitenlohner and D. Z. Freedman, "Positive energy in anti-de Sitter backgrounds and gauged extended supergravity”, Phys. Lett. B 115:3 (1982), 197-201. MR 83i:83055 Zbl 0606.53044

[Breitenlohner and Freedman 1982b] P. Breitenlohner and D. Z. Freedman, "Stability in gauged extended supergravity", Ann. Physics 144:2 (1982), 249-281. MR 84g:83044 Zbl 0606.53044

[Dafermos and Rodnianski 2005] M. Dafermos and I. Rodnianski, "A proof of Price's law for the collapse of a self-gravitating scalar field", Invent. Math. 162:2 (2005), 381-457. MR 2006i:83016 Zbl 1088.83008

[Dafermos and Rodnianski 2007] M. Dafermos and I. Rodnianski, "The wave equation on Schwarzschild-de Sitter space times", preprint, 2007. arXiv 07092766

[Dafermos and Rodnianski 2009] M. Dafermos and I. Rodnianski, "The red-shift effect and radiation decay on black hole spacetimes”, Comm. Pure Appl. Math. 62:7 (2009), 859-919. MR 2011b:83059 Zbl 1169.83008

[Fefferman and Graham 1985] C. Fefferman and C. R. Graham, Conformal invariants: The mathematical heritage of Élie Cartan (Lyon, 1984), Astérisque, Société Mathématique de France, Paris, 1985. MR 87g:53060 Zbl 0602.53007

[Graham and Lee 1991] C. R. Graham and J. M. Lee, "Einstein metrics with prescribed conformal infinity on the ball", $A d v$. Math. 87:2 (1991), 186-225. MR 92i:53041 Zbl 0765.53034

[Graham and Witten 1999] C. R. Graham and E. Witten, "Conformal anomaly of submanifold observables in AdS/CFT correspondence”, Nuclear Phys. B 546:1-2 (1999), 52-64. MR 2000h:81286 Zbl 0944.81046

[Graham and Zworski 2003] C. R. Graham and M. Zworski, "Scattering matrix in conformal geometry", Invent. Math. 152:1 (2003), 89-118. MR 2004c:58064 Zbl 1030.58022

[Holzegel 2010] G. Holzegel, "On the massive wave equation on slowly rotating Kerr-AdS spacetimes", Comm. Math. Phys. 294:1 (2010), 169-197. MR 2011d:58070 Zbl 1210.58023

[Hörmander 1971] L. Hörmander, "On the existence and the regularity of solutions of linear pseudo-differential equations", Enseignement Math. (2) 17 (1971), 99-163. MR 48 \#9458 Zbl 0224.35084

[Hörmander 1985] L. Hörmander, The analysis of linear partial differential operators, III: Pseudodifferential operators, Grundlehren der Mathematischen Wissenschaften 274, Springer, Berlin, 1985. MR 87d:35002a Zbl 0601.35001 
[Lebeau 1997] G. Lebeau, "Propagation des ondes dans les variétés à coins", Ann. Sci. École Norm. Sup. (4) 30:4 (1997), 429-497. MR 98d:58183 Zbl 0891.35072

[Mazzeo and Melrose 1987] R. R. Mazzeo and R. B. Melrose, "Meromorphic extension of the resolvent on complete spaces with asymptotically constant negative curvature", J. Funct. Anal. 75:2 (1987), 260-310. MR 89c:58133 Zbl 0636.58034

[Melrose 1993] R. B. Melrose, The Atiyah-Patodi-Singer index theorem, Research Notes in Mathematics 4, A K Peters, Wellesley, MA, 1993. MR 96g:58180 Zbl 0796.58050

[Melrose and Sjöstrand 1978] R. B. Melrose and J. Sjöstrand, "Singularities of boundary value problems, I", Comm. Pure Appl. Math. 31:5 (1978), 593-617. MR 58 \#11859 Zbl 0368.35020

[Melrose and Sjöstrand 1982] R. B. Melrose and J. Sjöstrand, "Singularities of boundary value problems, II", Comm. Pure Appl. Math. 35:2 (1982), 129-168. MR 83h:35120 Zbl 0546.35083

[Melrose and Taylor 1985] R. B. Melrose and M. E. Taylor, "Near peak scattering and the corrected Kirchhoff approximation for a convex obstacle", Adv. in Math. 55:3 (1985), 242-315. MR 86m:35095 Zbl 0591.58034

[Melrose et al. 2008] R. B. Melrose, A. S. Barreto, and A. Vasy, "Asymptotics of solutions of the wave equation on de SitterSchwarzschild space", preprint, 2008. arXiv 0811.2229

[Melrose et al. 2011] R. B. Melrose, A. S. Barreto, and A. Vasy, "Analytic continuation and semiclassical resolvent estimates on asymptotically hyperbolic spaces", preprint, 2011. arXiv 1103.3507

[Sá Barreto and Zworski 1997] A. Sá Barreto and M. Zworski, "Distribution of resonances for spherical black holes", Math. Res. Lett. 4:1 (1997), 103-121. MR 97m:83063 Zbl 0883.35120

[Sjöstrand 1980] J. Sjöstrand, "Propagation of analytic singularities for second order Dirichlet problems", Comm. Partial Differential Equations 5:1 (1980), 41-93. MR 81e:35031a Zbl 0458.35026

[Taylor 1976] M. E. Taylor, "Grazing rays and reflection of singularities of solutions to wave equations", Comm. Pure Appl. Math. 29:1 (1976), 1-38. MR 53 \#1035 Zbl 0318.35009

[Taylor 1996] M. E. Taylor, Partial differential equations: Basic theory, Texts in Applied Mathematics 23, Springer, New York, 1996. MR 98b:35002a Zbl 0869.35001

[Vasy 2005] A. Vasy, "Propagation of singularities for the wave equation on manifolds with corners", in Séminaire: Équations aux Dérivées Partielles, 2004-2005 (Exposé 20), École Polytech., Palaiseau, 2005. MR 2006j:58045

[Vasy 2008a] A. Vasy, "A correction to 'Propagation of singularities for the wave equation on manifolds with corners", preprint, 2008, Available at math.stanford.edu/ andras/psmc-corr.pdf.

[Vasy 2008b] A. Vasy, "Diffraction by edges", Modern Phys. Lett. B 22:23 (2008), 2287-2328. MR 2010b:58039 Zbl 1159. 78302

[Vasy 2008c] A. Vasy, "Propagation of singularities for the wave equation on manifolds with corners", Ann. of Math. (2) 168:3 (2008), 749-812. MR 2009i:58037 Zbl 1171.58007

[Vasy 2010a] A. Vasy, "Diffraction at corners for the wave equation on differential forms", Comm. Partial Differential Equations 35:7 (2010), 1236-1275. MR 2012a:58051 Zbl 1208.58026

[Vasy 2010b] A. Vasy, "The wave equation on asymptotically de Sitter-like spaces", Adv. Math. 223:1 (2010), 49-97. MR 2011i:58046 Zbl 1191.35064

[Witten 1998] E. Witten, "Anti de Sitter space and holography", Adv. Theor. Math. Phys. 2:2 (1998), 253-291. MR 99e:81204c Zbl 0914.53048

[Yagdjian and Galstian 2009] K. Yagdjian and A. Galstian, "The Klein-Gordon equation in anti-de Sitter spacetime", Rend. Semin. Mat. Univ. Politec. Torino 67:2 (2009), 271-292. MR 2011d:35282 Zbl 1184.35109

Received 23 Dec 2009. Revised 11 Oct 2010. Accepted 22 Dec 2010.

ANDRÁs VASY: andras@math.stanford.edu

Department of Mathematics, Stanford University, Stanford, CA 94305-2125, United States

http://math.stanford.edu/ andras/ 


\title{
Analysis \& PDE
}

\author{
msp.berkeley.edu/apde
}

EDITORS

EDITOR-IN-CHIEF

Maciej Zworski

University of California

Berkeley, USA

BOARD OF EDITORS

\begin{tabular}{|c|c|c|c|}
\hline Michael Aizenman & $\begin{array}{l}\text { Princeton University, USA } \\
\text { aizenman@math.princeton.edu }\end{array}$ & Nicolas Burq & $\begin{array}{l}\text { Université Paris-Sud 11, France } \\
\text { nicolas.burq@math.u-psud.fr }\end{array}$ \\
\hline Luis A. Caffarelli & $\begin{array}{l}\text { University of Texas, USA } \\
\text { caffarel@ math.utexas.edu }\end{array}$ & un-Yung Alice Chang & $\begin{array}{l}\text { Princeton University, USA } \\
\text { chang@ math.princeton.edu }\end{array}$ \\
\hline Michael Christ & $\begin{array}{l}\text { University of California, Berkeley, USA } \\
\text { mchrist@math.berkeley.edu }\end{array}$ & Charles Fefferman & $\begin{array}{l}\text { Princeton University, USA } \\
\text { cf@math.princeton.edu }\end{array}$ \\
\hline Ursula Hamenstaedt & $\begin{array}{l}\text { Universität Bonn, Germany } \\
\text { ursula@math.uni-bonn.de }\end{array}$ & Nigel Higson & $\begin{array}{l}\text { Pennsylvania State Univesity, USA } \\
\text { higson@math.psu.edu }\end{array}$ \\
\hline Vaughan Jones & $\begin{array}{l}\text { University of California, Berkeley, USA } \\
\text { vfr@math.berkeley.edu }\end{array}$ & Herbert Koch & $\begin{array}{l}\text { Universität Bonn, Germany } \\
\text { koch@math.uni-bonn.de }\end{array}$ \\
\hline Izabella Laba & $\begin{array}{l}\text { University of British Columbia, Canada } \\
\text { ilaba@math.ubc.ca }\end{array}$ & Gilles Lebeau & $\begin{array}{l}\text { Université de Nice Sophia Antipolis, France } \\
\text { lebeau@unice.fr }\end{array}$ \\
\hline László Lempert & $\begin{array}{l}\text { Purdue University, USA } \\
\text { lempert@math.purdue.edu }\end{array}$ & Richard B. Melrose & $\begin{array}{l}\text { Massachussets Institute of Technology, USA } \\
\text { rbm@math.mit.edu }\end{array}$ \\
\hline Frank Merle & $\begin{array}{l}\text { Université de Cergy-Pontoise, France } \\
\text { Frank.Merle@u-cergy.fr }\end{array}$ & William Minicozzi II & $\begin{array}{l}\text { Johns Hopkins University, USA } \\
\text { minicozz@ math.jhu.edu }\end{array}$ \\
\hline Werner Müller & $\begin{array}{l}\text { Universität Bonn, Germany } \\
\text { mueller@math.uni-bonn.de }\end{array}$ & Yuval Peres & $\begin{array}{l}\text { University of California, Berkeley, USA } \\
\text { peres@stat.berkeley.edu }\end{array}$ \\
\hline Gilles Pisier & $\begin{array}{l}\text { Texas A\&M University, and Paris } 6 \\
\text { pisier@math.tamu.edu }\end{array}$ & Tristan Rivière & $\begin{array}{l}\text { ETH, Switzerland } \\
\text { riviere@math.ethz.ch }\end{array}$ \\
\hline Igor Rodnianski & $\begin{array}{l}\text { Princeton University, USA } \\
\text { irod@math.princeton.edu }\end{array}$ & Wilhelm Schlag & $\begin{array}{l}\text { University of Chicago, USA } \\
\text { schlag@math.uchicago.edu }\end{array}$ \\
\hline Sylvia Serfaty & $\begin{array}{l}\text { New York University, USA } \\
\text { serfaty@ cims.nyu.edu }\end{array}$ & Yum-Tong Siu & $\begin{array}{l}\text { Harvard University, USA } \\
\text { siu@math.harvard.edu }\end{array}$ \\
\hline Terence Tao & $\begin{array}{l}\text { University of California, Los Angeles, USA } \\
\text { tao@math.ucla.edu }\end{array}$ & Michael E. Taylor & $\begin{array}{l}\text { Univ. of North Carolina, Chapel Hill, USA } \\
\text { met@math.unc.edu }\end{array}$ \\
\hline Gunther Uhlmann & $\begin{array}{l}\text { University of Washington, USA } \\
\text { gunther@math.washington.edu }\end{array}$ & András Vasy & $\begin{array}{l}\text { Stanford University, USA } \\
\text { andras@math.stanford.edu }\end{array}$ \\
\hline an Virgil Voiculescu & $\begin{array}{l}\text { University of California, Berkeley, USA } \\
\text { dvv@ math.berkeley.edu }\end{array}$ & Steven Zelditch & $\begin{array}{l}\text { Northwestern University, USA } \\
\text { zelditch@math.northwestern.edu }\end{array}$ \\
\hline
\end{tabular}

\section{PRODUCTION}

contact@msp.org

Silvio Levy, Scientific Editor

Sheila Newbery, Senior Production Editor

See inside back cover or msp.berkeley.edu/apde for submission instructions.

The subscription price for 2012 is US \$140/year for the electronic version, and \$240/year for print and electronic. Subscriptions, requests for back issues from the last three years and changes of subscribers address should be sent to Mathematical Sciences Publishers, Department of Mathematics, University of California, Berkeley, CA 94720-3840, USA.

Analysis \& PDE, at Mathematical Sciences Publishers, Department of Mathematics, University of California, Berkeley, CA 94720-3840 is published continuously online. Periodical rate postage paid at Berkeley, CA 94704, and additional mailing offices.

APDE peer review and production are managed by EditFLOW ${ }^{\mathrm{TM}}$ from Mathematical Sciences Publishers.

PUBLISHED BY

mathematical sciences publishers

http://msp.org/

A NON-PROFIT CORPORATION

Typeset in IATEX

Copyright $(2012$ by Mathematical Sciences Publishers 


\section{ANALYSIS \& PDE}

\section{Volume $5 \quad$ No. $1 \quad 2012$}

A characterization of two weight norm inequalities for maximal singular integrals with one 1 doubling measure

Michael LACEy, ERic T. SAWyer and IgNACio URiarte-Tuero

Energy identity for intrinsically biharmonic maps in four dimensions

PETER HORNung and ROger MOSER

The wave equation on asymptotically anti de Sitter spaces

ANDRÁS VASY

Small data scattering and soliton stability in $\dot{H}^{-1 / 6}$ for the quartic KdV equation

HERBERT KOCH and JEREMY L. MARZUOLA

A remark on barely $\dot{H}^{s_{p}}$-supercritical wave equations

199

TRISTAN ROY 